\title{
SOUNDNESS OF UNRAVELINGS FOR CONDITIONAL TERM REWRITING SYSTEMS VIA ULTRA-PROPERTIES RELATED TO LINEARITY*
}

\author{
NAOKI NISHIDA, MASAHIKO SAKAI, AND TOSHIKI SAKABE
}

Graduate School of Information Science, Nagoya University

e-mail address: \{nishida, sakai, sakabe\}@is.nagoya-u.ac.jp

\begin{abstract}
Unravelings are transformations from conditional term rewriting systems (CTRSs) into unconditional term rewriting systems (TRSs) over extended signatures. They are complete, but in general, not sound w.r.t. reduction. Here, soundness w.r.t. reduction for a CTRS means that for every term over the original signature of the CTRS, if the corresponding unraveled TRS reduces the term to a term over the original signature, then so does the original CTRS. In this paper, we show that an optimized variant of Ohlebusch's unraveling for deterministic CTRSs is sound w.r.t. reduction if the corresponding unraveled TRSs are left-linear, or both right-linear and non-erasing. Then, we show that soundness of the variant implies soundness of Ohlebusch's unraveling, and show that soundness of Marchiori's unravelings for join and normal CTRSs also implies soundness of Ohlebusch's unraveling. Finally, we show that soundness of a transformation proposed by Şerbănuţă and Roşu for deterministic CTRSs implies soundness of Ohlebusch's unraveling.
\end{abstract}

\section{INTRODUCTION}

Unravelings are transformations from conditional term rewriting systems (CTRSs) into unconditional term rewriting systems (TRSs) over extended signatures of the original signatures for the CTRSs. They are complete w.r.t. reduction sequences of the original CTRSs [15], i.e., for every derivation of the CTRSs, there exists a corresponding derivation of the unraveled TRSs. In this respect, the unraveled TRSs are over-approximations of the original CTRSs w.r.t. reduction, and the unraveled TRSs are useful for analyzing the properties of the original CTRSs, such as syntactic properties, modularity, and operational termination since TRSs are in general much easier to handle than CTRSs.

Marchiori proposed unravelings for join and normal CTRSs in order to analyze ultraproperties and modularity of the CTRSs [15, and he also proposed an unraveling for deterministic CTRSs (DCTRSs) [16]. The transformation technique used in his unravelings originates from [4, 8]. Afterwards, Ohlebusch presented an improved variant of Marchiori's

1998 ACM Subject Classification: F.4.2.

Key words and phrases: conditional term rewriting, program transformation.

* This research has been partially supported by MEXT KAKENHI \#17700009, \#20300010 and \#21700011, and Kayamori Foundation of Informational Science Advancement. 
unraveling for DCTRSs in order to analyze termination of logic programs [25] - Marchiori's and Ohlebusch's unravelings are called sequential unravelings [10]. Termination of the unraveled TRSs is a sufficient condition for proving operational termination of the original CTRSs [14. Later, a variant of Ohlebusch's unraveling was proposed in both [18] and [7]. This variant is sometimes called optimized, in the sense that the variable-carrying arguments of $U$ symbols introduced by means of the application of the unraveling are optimized, i.e., U symbols propagate only values received by variables that are referred later.

Although the mechanism of unconditional rewriting is much simpler than that of conditional rewriting, the reduction of the unraveled TRS has never been used instead of the original CTRS in order to reduce terms over the original signature, until being used in program inversion methods [18, 22, 23] described later. This is because unravelings are not sound w.r.t. reduction in general [15, 25] while they are complete. Here, soundness w.r.t. reduction (simply, soundness) for a CTRS means that, for every term over the original signature of the CTRS, if the unraveled TRS reduces the term to a term over the original signature, then so does the original CTRS [15]. Several studies have been made on soundness conditions of unravelings - some syntactic properties and particular reduction strategies for the unraveled TRSs. Marchiori showed that his unraveling for normal CTRSs is sound for left-linear ones [15], and he also showed that his unraveling for DCTRSs is sound for DCTRSs that are semi-linear or confluent [16]. Nishida et al. showed that the combined reduction restriction of the membership condition [34] and context-sensitive condition [13] determined by means of the application of the optimized unraveling is sufficient for soundness [22]. Later, Schernhammer and Gramlich showed that the same context-sensitive condition without the membership condition is sufficient for soundness of Ohlebusch's unraveling [28, 29] and Gmeiner et al. showed that Marchiori's unraveling for normal CTRSs is sound for confluent, non-erasing, or weakly left-linear ones, and they presented some properties that are not sufficient for soundness [9].

As another kind of transformation from CTRSs to TRSs, Şerbănuţă and Roşu proposed a complete transformation (SR transformation) from strongly or syntactically DCTRSs into TRSs [30, 31]. The SR transformation is sound if the DCTRSs are semi-linear or confluent, where function symbols in the original signatures are completely extended by increasing the arities of some function symbols. The SR transformation is based on Viry's approach 35. that is another direction of developing transformations from CTRSs into TRSs, and that has been further studied in [1, 27]. The SR transformation provides computationally equivalent TRSs to the original DCTRSs if the original DCTRSs are operationally terminating and either semi-linear or (ground) confluent. On the other hand, the theoretical relationship between the SR transformation and the existing unravelings has never been discussed.

In this paper, we show two sufficient conditions of DCTRSs for soundness of the optimized unraveling: one condition is ultra-left-linearity, i.e., that the unraveled TRSs are left-linear, and the second condition is the combination of ultra-right-linearity and ultranon-erasingness, i.e., that the unraveled TRSs are right-linear and non-erasing. We also provide necessary and sufficient conditions of DCTRSs under which the corresponding unraveled TRSs are left-linear, right-linear, and non-erasing, respectively. All the conditions are syntactic and it is decidable whether a DCTRS satisfies the conditions. Moreover, we show that soundness of the optimized unraveling implies soundness of Ohlebusch's unraveling, i.e., if the optimized unraveling is sound for a DCTRS, then so is Ohlebusch's 
unraveling. Finally, we show that soundness of the existing unravelings and the SR transformation respectively imply soundness of Ohlebusch's unraveling. This paper is different from the preliminary version [24] in that we present

- abstract comparison methods for soundness of two transformations from CTRSs into TRSs (Lemmas 5.3, 5.20 and Theorem 6.2),

- a comparison with other unravelings for join and normal DCTRSs (Subsection 5.3) in terms of soundness, and

- a comparison with the SR transformation (Section 6) in terms of soundness.

The optimized unraveling has been employed in the (full or partial) program inversion methods for constructor TRSs [18, 22, 23]. The methods first transform a constructor TRS into a DCTRS that defines (full or partial) inverses of functions defined in the constructor TRS, and then unravel the DCTRS into a TRS (see Example 3.2). The resulting TRS may have extra variables since the intermediate DCTRS may have extra variables that occur on the right-hand side, but not in the conditional part. For this reason, this paper allows TRSs to have extra variables. In applying a rewrite rule, extra variables of the rule are allowed to be instantiated with arbitrary terms. Since many instantiated terms of extra variables are meaningless and sometimes cause non-termination, we limit reduction sequences to meaningful ones by giving a restriction to reduction sequences of the resulting TRS. The restriction is $E V$-safeness [20, 18, 21] that is a relaxed variant of the basicness property [11, 17. of reduction sequences: when a TRS has extra variables, any redex introduced by means of extra variables is not reduced anywhere in the reduction sequences. In this paper, we discuss soundness of unravelings w.r.t. EV-safe derivations of the unraveled TRSs.

It has been shown that the optimized unraveling is sound for the intermediate DCTRSs of the inversion methods in [18, 22, 23, where conditional rules of the intermediate DCTRSs are of the restricted form: $l \rightarrow r \Leftarrow s_{1} \rightarrow t_{1} ; \ldots ; s_{k} \rightarrow t_{k}$ where $r, t_{1}, \ldots, t_{k}$ are non-variable constructor terms and $s_{1}, \ldots, s_{k}$ are rooted by defined symbols. Although the optimized unraveling is known to be sound for the intermediate DCTRSs, studies on soundness conditions of the (optimized) unraveling would be useful when the intermediate DCTRSs are further transformed into more relaxed forms, e.g., DCTRSs obtained by removing a unary tuple symbol $\mathrm{tp}_{1}$ (see Example 4.10). Roughly speaking, in applying the inversion method, the resulting TRS is often right-linear if the input constructor TRS is left-linear. Moreover, the resulting TRS is non-erasing if the input constructor TRS is fully inverted, and, in addition, the resulting TRS has no extra variable if the input is non-erasing. Note that injective functions are often defined by non-erasing TRSs and the class of injective functions is the most interesting as an object of program inversion. For the reasons mentioned above, the sufficient conditions shown in this paper can be used to guarantee that the resulting TRSs of the inversion method for left-linear constructor TRSs are definitely inverses of the constructor TRSs (see Example 4.13).

As mentioned previously, Ohlebusch's unraveling is sound for any DCTRS if we introduce the particular context-sensitive restriction to the reduction of the corresponding unraveled TRSs. Since recently context-sensitive reduction has been well investigated (e.g., techniques to prove context-sensitive termination) and its interpreter can be easily implemented, the unraveled TRSs with the particular context-sensitivity can be used instead of the original CTRSs to completely reduce terms over the original signature to terms over the original signature. However, sufficient (syntactic) properties for soundness without the 
restriction to the reduction are useful for the use of the unraveled TRSs instead of the original CTRSs since context-sensitivity makes the reduction more complicated than ordinary reduction. Moreover, if the unraveling used in [18, 22, 23] is sound for the resulting TRS obtained by the inversion method without context-sensitivity, then we can apply the restricted version of completion [19] to the resulting TRS to make the resulting TRS convergent or to provide useful information for transforming the intermediate DCTRS into an equivalent functional program. For these reasons, soundness of unravelings without any restriction to the reduction is meaningful in order to employ the reduction of the unraveled TRSs instead of the original CTRSs.

In summary, the main contribution of this paper is to show the following:

- the optimized unraveling is sound for a DCTRS that is ultra-left-linear, or both ultraright-linear and ultra-non-erasing (Theorems 4.3, 4.9),

- soundness of the existing unravelings and the SR transformation respectively implies soundness of Ohlebusch's unraveling (Corollary 5.5 and Theorems 5.21, 6.12), and

- abstract comparison methods for soundness of two transformations from CTRSs into TRSs (Lemmas 5.3, 5.20 and Theorem 6.2).

All the soundness conditions are summarized at the end of this paper (Table 1 in Subsection 6.4).

This paper is organized as follows. In Section 2, we recall basic notions and notations of term rewriting. In Section 3, we review the existing unravelings for DCTRSs, and present syntactic properties of DCTRSs for some ultra-properties. In Section 4, we show that the optimized unraveling is sound for a DCTRS if the corresponding unraveled TRS is left-linear, or both right-linear and non-erasing. In Section 5, we show that soundness of the existing unravelings for join, normal, and deterministic CTRSs respectively implies soundness of Ohlebusch's unraveling. In Section 6, we compare soundness of Ohlebusch's unraveling with soundness of the SR transformation. In Section 7 , we briefly describe related work and summarize soundness conditions of unravelings and the SR transformation. In Section 8, we conclude this paper and briefly describe future work on unravelings. Proofs of some technical results are included in the appendix.

\section{Preliminaries}

In this section, we recall basic notions and notations of term rewriting [3, 26].

Let $\rightarrow_{L}$ be a binary relation (over a set of $A$ ) with a label $L$. The reflexive closure of $\rightarrow_{L}$ is denoted by $\rightarrow_{L}^{\bar{L}}$, the transitive closure of $\rightarrow_{L}$ by $\rightarrow_{L}^{+}$, and the reflexive and transitive closure of $\rightarrow_{L}$ by $\rightarrow_{L}^{*}$. The joinability relation w.r.t. $\rightarrow_{L}$ is denoted by $\downarrow_{L}: \downarrow_{L}=\rightarrow_{L}^{*} \cdot \leftarrow_{L}^{*}$. An element $a \in A$ is called a normal form w.r.t. $\rightarrow_{L}$ (or w.r.t. $L$ ) if there exists no element $b \in A$ such that $a \rightarrow_{L} b$.

Throughout the paper, we use $\mathcal{V}$ as a countably infinite set of variables. Let $\mathcal{F}$ be a signature, a finite set of function symbols each of which has its own fixed arity, and arity $(f)$ be the arity of function symbol $f$. The set of terms over $\mathcal{F}$ and $\mathcal{V}$ is denoted by $\mathcal{T}(\mathcal{F}, \mathcal{V})$, and the set of variables appearing in any of terms $t_{1}, \ldots, t_{n}$ is denoted by $\mathcal{V} a r\left(t_{1}, \ldots, t_{n}\right)$. A term $t$ is called ground if $\operatorname{Var}(t)=\emptyset$. A term is called linear if any variable occurs in the term at most once, and called linear w.r.t. a variable if the variable appears at most once in $t$. The set of positions of term $t$ is denoted by $\mathcal{P} o s(t)$. The set of positions for function symbols in $t$ is denoted by $\operatorname{Pos}_{\mathcal{F}}(t)$, and the set of positions for variables in $t$ is denoted by $\mathcal{P o s}_{\mathcal{V}}(t)$. For term $t$ and position $p$ of $t$, the notation $\left.t\right|_{p}$ represents the subterm of $t$ at position $p$. 
The function symbol at the root position $\varepsilon$ of term $t$ is denoted by $\operatorname{root}(t)$. Given an $n$-hole context $C\left[\right.$ ] with parallel positions $p_{1}, \ldots, p_{n}$, the notation $C\left[t_{1}, \ldots, t_{n}\right]_{p_{1}, \ldots, p_{n}}$ represents the term obtained by replacing hole $\square$ at position $p_{i}$ with term $t_{i}$ for all $1 \leq i \leq n$. We may omit the subscription $p_{1}, \ldots, p_{n}$ from $C[\ldots]_{p_{1}, \ldots, p_{n}}$. For positions $p$ and $p^{\prime}$ of a term, we write $p^{\prime} \geq p$ if $p$ is a prefix of $p^{\prime}$ (i.e., there exists a sequence $q$ such that $p q=p^{\prime}$ ). Moreover, we write $p^{\prime}>p$ if $p$ is a proper prefix of $p^{\prime}$.

The domain and range of a substitution $\sigma$ are denoted by $\mathcal{D} o m(\sigma)$ and $\mathcal{R} a n(\sigma)$, respectively. We may denote $\sigma$ by $\left\{x_{1} \mapsto t_{1}, \ldots, x_{n} \mapsto t_{n}\right\}$ if $\mathcal{D o m}(\sigma)=\left\{x_{1}, \ldots, x_{n}\right\}$ and $\sigma\left(x_{i}\right)=$ $t_{i}$ for all $1 \leq i \leq n$. For a signature $\mathcal{F}$, the set of substitutions whose ranges are over $\mathcal{F}$ and $\mathcal{V}$ is denoted by $\operatorname{Sub}(\mathcal{F}, \mathcal{V}): \mathcal{S} u b(\mathcal{F}, \mathcal{V})=\{\sigma \mid \mathcal{R} a n(\sigma) \subseteq \mathcal{T}(\mathcal{F}, \mathcal{V})\}$. For a substitution $\sigma$ and a term $t$, the application $\sigma(t)$ of $\sigma$ to $t$ is abbreviated to $t \sigma$, and $t \sigma$ is called an instance of $t$. Given a set $X$ of variables, $\left.\sigma\right|_{X}$ denotes the restricted substitution of $\sigma$ w.r.t. $X:\left.\sigma\right|_{X}$ $=\{x \mapsto x \sigma \mid x \in \operatorname{Dom}(\sigma) \cap X\}$. The composition $\sigma \theta$ of substitutions $\sigma$ and $\theta$ is defined as $x(\sigma \theta)=(x \sigma) \theta$.

A conditional rewrite rule over a signature $\mathcal{F}$ is a triple $(l, r, c)$, denoted by $l \rightarrow r \Leftarrow c$, such that the left-hand side $l$ is a non-variable term in $\mathcal{T}(\mathcal{F}, \mathcal{V})$, the right-hand side $r$ is a term in $\mathcal{T}(\mathcal{F}, \mathcal{V})$, and the conditional part $c$ is a sequence $s_{1} \approx t_{1} ; \ldots ; s_{k} \approx t_{k}$ of term pairs $(k$ $\geq 0)$ where all of $s_{1}, t_{1}, \ldots, s_{k}, t_{k}$ are terms in $\mathcal{T}(\mathcal{F}, \mathcal{V})$. In particular, a conditional rewrite rule is called unconditional if the conditional part is the empty sequence (i.e., $k=0$ ), and we may abbreviate it to $l \rightarrow r$. The conditional rewrite rule is called extended if the condition " $l \notin \mathcal{V} "$ is not imposed. We sometimes attach a unique label $\rho$ to the conditional rewrite rule $l \rightarrow r \Leftarrow c$ by denoting $\rho: l \rightarrow r \Leftarrow c$, and we use the label to refer to the rewrite rule. The sets of variables in $c$ and $\rho$ are denoted by $\mathcal{V} a r(c)$ and $\mathcal{V} a r(\rho)$, respectively: $\mathcal{V} a r\left(s_{1} \approx t_{1} ; \ldots ; s_{k} \approx t_{k}\right)=\operatorname{Var}\left(s_{1}, t_{1}, \ldots, s_{k}, t_{k}\right)$ and $\mathcal{V} a r(\rho)=\operatorname{Var}(l, r) \cup \mathcal{V} a r(c)$. A variable occurring in $r$ or $c$ is called an extra variables of $\rho$ if it does not occur in $l$. The set of extra variables of $\rho$ is denoted by $\mathcal{E} \operatorname{Var}(\rho): \mathcal{E} \mathcal{V} a r(\rho)=(\mathcal{V} a r(r) \cup \mathcal{V} a r(c)) \backslash \mathcal{V} a r(l)$.

A conditional term rewriting system (CTRS) over a signature $\mathcal{F}$ is a set of conditional rules over $\mathcal{F}$. In particular, a CTRS is called an $E V$-TRS if all of its rules are unconditional, and called an extended CTRS (eCTRS) if the condition " $l \notin \mathcal{V}$ " of conditional rewrite rules $l \rightarrow r \Leftarrow c$ is not imposed. Moreover, a CTRS is called an (unconditional) term rewriting system (TRS) if every rule $l \rightarrow r \Leftarrow c$ in the CTRS is unconditional and satisfies $\mathcal{V} a r(l) \supseteq$ $\operatorname{Var}(r)$. Note that an eCTRS is called an $e T R S$ if all of its rules are unconditional. The underlying unconditional system of a CTRS $R$ is denoted by $R_{u}: R_{u}=\{l \rightarrow r \mid l \rightarrow r \Leftarrow$ $c \in R\}$.

A CTRS $R$ is called oriented if the symbol $\approx$ in the conditions of its rewrite rules is interpreted as reachability: the reduction relation of $R$ is defined as $\rightarrow_{R}=\bigcup_{n \geq 0} \rightarrow_{(n), R}$ where

- $\rightarrow(0), R=\emptyset$, and

- $\rightarrow_{(i+1), R}=\rightarrow_{(i), R} \cup\left\{\left(C[l \sigma]_{p}, C[l \sigma]_{p}\right) \mid \rho: l \rightarrow r \Leftarrow s_{1} \approx t_{1} ; \ldots ; s_{k} \approx t_{k} \in R, s_{1} \sigma \rightarrow_{(i), R}^{*}\right.$ $\left.t_{1} \sigma, \ldots, s_{k} \sigma \rightarrow_{(i), R}^{*} t_{k} \sigma\right\}$ for $i \geq 0$.

Rewrite rules $l \rightarrow r \Leftarrow s_{1} \approx t_{1} ; \ldots ; s_{k} \approx t_{k}$ of oriented CTRSs are written as $l \rightarrow r \Leftarrow s_{1} \rightarrow$ $t_{1} ; \ldots ; s_{k} \rightarrow t_{k}$. To specify the applied rule $\rho$ and the position $p$ where $\rho$ is applied, we may write $\rightarrow_{p, \rho}$ or $\rightarrow_{p, R}$ instead of $\rightarrow_{R}$. Moreover, we may write $\rightarrow_{>\varepsilon, R}$ instead of $\rightarrow_{p, R}$ if $p>\varepsilon$. The parallel reduction $\rightrightarrows_{R}$ is defined as $\rightrightarrows_{R}=\left\{\left(C\left[s_{1}, \ldots, s_{n}\right]_{p_{1}, \ldots, p_{n}}, C\left[t_{1}, \ldots, t_{n}\right]_{p_{1}, \ldots, p_{n}}\right) \mid\right.$ $\left.s_{1} \rightarrow_{R} t_{1}, \ldots, s_{n} \rightarrow_{R} t_{n}\right\}$. To specify the positions $p_{1}, \ldots, p_{n}$ in the definition, we may write $\rightrightarrows\left\{p_{1}, \ldots, p_{n}\right\}, R$ instead of $\rightrightarrows_{R}$, and we may write $\rightrightarrows_{>\varepsilon, R}$ instead of $\rightrightarrows_{R}$ if $p_{i}>\varepsilon$ for all 
$1 \leq i \leq n$. Moreover, for a set $P$ of parallel positions, we may write $\rightrightarrows \geq P, R$ instead of $\rightrightarrows R$ if, for each position $p_{i} \in\left\{p_{1}, \ldots, p_{n}\right\}$, there exists a position $p \in P$ such that $p \leq p_{i}$.

For an eCTRS $R$, a substitution $\sigma$ is called normalized w.r.t. $R$ if $x \sigma$ is a normal form w.r.t. $R$ for every variable $x \in \mathcal{D} o m(\sigma)$.

An (extended) conditional rewrite rule $l \rightarrow r \Leftarrow c$ is called

- left-linear (LL) if $l$ is linear,

- right-linear (RL) if $r$ is linear,

- non-erasing (NE) if $\mathcal{V} a r(l) \subseteq \mathcal{V} a r(r)$,

- non-collapsing or non-right-variable (non-RV) if the right-hand side $r$ is not a variable, and

- non-left-variable (non-LV) if $l$ is not a variable.

For a syntactic property $P$ of conditional rewrite rules, we say that an eCTRS has the property $P$ if all of its rules have the property $P$, e.g., an eCTRS is called left-linear (LL) if all of its rules are LL. Note that a non-LV eCTRS is a CTRS.

An (extended) conditional rewrite rule $\rho: l \rightarrow r \Leftarrow s_{1} \rightarrow t_{1} ; \ldots ; s_{k} \rightarrow t_{k}$ is called deterministic if $\operatorname{Var}\left(s_{i}\right) \subseteq \mathcal{V}$ ar $\left(l, t_{1}, \ldots, t_{i-1}\right)$ for all $1 \leq i \leq k$. An (e)CTRS is called deterministic, an (e)DCTRS for short, if all of its rules are deterministic. Conditional rule $\rho$ is classified according to the distribution of variables in the rule as follows:

- Type 1 if $\operatorname{Var}\left(r, s_{1}, t_{1}, \ldots, s_{k}, t_{k}\right) \subseteq \mathcal{V} a r(l)$,

- Type 2 if $\mathcal{V} a r(r) \subseteq \mathcal{V} a r(l)$,

- Type 3 if $\mathcal{V} a r(r) \subseteq \mathcal{V} a r\left(l, s_{1}, t_{1}, \ldots, s_{k}, t_{k}\right)$, and

- Type 4 otherwise.

An (eD)CTRS is called an $i$-(eD)CTRS if all of its rules are of Type $i$. An eDCTRS $R$ is called normal (or a normal CTRS) if, for every rule $l \rightarrow r \Leftarrow s_{1} \rightarrow t_{1} ; \ldots ; s_{k} \rightarrow t_{k} \in R$, all of $t_{1}, \ldots, t_{k}$ are ground normal forms w.r.t. $R_{u}$.

Let $R$ be a CTRS over a signature $\mathcal{F}$. The sets of defined symbols and constructors of $R$ are denoted by $\mathcal{D}_{R}$ and $\mathcal{C}_{R}$, respectively: $\mathcal{D}_{R}=\{\operatorname{root}(l) \mid l \rightarrow r \Leftarrow c \in R\}$ and $\mathcal{C}_{R}=$ $\mathcal{F} \backslash \mathcal{D}_{R}$. Terms in $\mathcal{T}\left(\mathcal{C}_{R}, \mathcal{V}\right)$ are constructor terms of $R$. $R$ is called a constructor system if all proper subterms of the left-hand sides in $R$ are constructor terms of $R$.

Let $R$ be a CTRS. Two conditional rewrite rules $l_{1} \rightarrow r_{1} \Leftarrow c_{1}$ and $l_{2} \rightarrow r_{2} \Leftarrow c_{2}$ in $R$ are called overlapping if there exists a context $C[]$ and a non-variable term $t$ such that $l_{2}=$ $C[t]$ and $l_{1}$ and $t$ are unifiable, where we assume w.l.o.g. that these rules share no variable. Then, a conditional pair of terms $\left(\left(C\left[r_{1}\right]\right) \theta, r_{2} \theta\right) \Leftarrow c_{1} \theta ; c_{2} \theta$ is called a critical pair of $R$ where $\theta$ is a most general unifier of $l_{1}$ and $t$. A critical pair $(s, t) \Leftarrow c$ is called trivial if $s$ $=t$, and called infeasible if for any substitution $\sigma, c$ contains a condition $u \rightarrow v$ such that $u \sigma \not A_{R}^{*} v \sigma[12](c f .,[26])$.

Let $\mathcal{F}_{1}, \mathcal{F}_{2}$ be signatures, $\mathcal{G} \subseteq \mathcal{F}_{1} \cap \mathcal{F}_{2}$, and $\rightarrow_{1}, \rightarrow_{2}$ be binary relations on terms in $\mathcal{T}\left(\mathcal{F}_{1}, \mathcal{V}\right)$ and $\mathcal{T}\left(\mathcal{F}_{2}, \mathcal{V}\right)$, respectively. We say that $\rightarrow_{1} \subseteq \rightarrow_{2}$ on terms in $\mathcal{T}(\mathcal{G}, \mathcal{V})$ if, for all terms $s, t \in \mathcal{T}(\mathcal{G}, \mathcal{V}), s \rightarrow_{2} t$ whenever $s \rightarrow_{1} t$.

\section{UnRavelings for Deterministic CTRSs}

In this section, we first recall unravelings for DCTRSs, and then show some syntactic properties of DCTRSs, that are related to the syntactic properties of the unraveled TRSs. The unravelings and some results in this section are straightforwardly extended to eDCTRSs. 
We first recall the notion of unravelings. A computable transformation $U$ from eCTRSs into eTRSs is called an unraveling if for every eCTRS $R$, we have $\rightarrow_{R} \subseteq \rightarrow_{U(R)}^{*}$ and $U\left(R^{\prime} \cup R\right)=R^{\prime} \cup U(R)$ whenever $R^{\prime}$ is an eTRS [15, 16] 1] Unraveling $U$ is called tidy if it has compositionality $\left(U\left(R_{1} \cup R_{2}\right)=U\left(R_{1}\right) \cup U\left(R_{2}\right)\right)$, finiteness (if $R$ is finite, then so is $U(R)$ ), and emptiness (if $R$ is empty, then so is $U(R)$ ) [15]. Let $R$ be an eCTRS over a signature $\mathcal{F}$, and $\Rightarrow_{U(R)}$ be a subrelation of $\rightarrow_{U(R)}$. $U$ is called sound w.r.t. reduction for $R$ w.r.t. $\Rightarrow_{U(R)}$ (simulation-sound [21, 22], or simply sound for $R$ w.r.t. $\left.\Rightarrow_{U(R)}\right)$ if $\Rightarrow_{U(R)}^{*}$ $\subseteq \rightarrow_{R}^{*}$ on terms in $\mathcal{T}(\mathcal{F}, \mathcal{V})$ (i.e., for all terms $s, t$ in $\mathcal{T}(\mathcal{F}, \mathcal{V})$, if $s \Rightarrow_{U(R)}^{*} t$, then $s \rightarrow_{R}^{*} t$ ). $U$ is called complete w.r.t. reduction for $R$ w.r.t. $\Rightarrow_{U(R)}$ (or simply complete for $R$ w.r.t. $\left.\Rightarrow_{U(R)}\right)$ if $\rightarrow_{R}^{*} \subseteq \Rightarrow_{U(R)}^{*}$ on terms in $\mathcal{T}(\mathcal{F}, \mathcal{V})$. We omit "w.r.t. $\rightarrow_{U(R)}$ " if $\Rightarrow_{\mathbb{U}(R)}=\rightarrow_{U(R)}$.

Next, we recall an unraveling for DCTRSs, proposed by Ohlebusch [25] that is a natural improvement of Marchiori's unraveling [16]. For a finite set $T=\left\{t_{1}, \ldots, t_{n}\right\}$, given some fixed ordering $\prec$ such that $t_{1} \prec \cdots \prec t_{n}, \vec{T}$ denotes the unique sequence $t_{1}, \ldots, t_{n}$ of elements in $T$.

Definition 3.1 (unraveling $\mathbb{U}[25]$ ). Let $R$ be an eDCTRS over a signature $\mathcal{F}$. For every conditional rule $\rho: l \rightarrow r \Leftarrow s_{1} \rightarrow t_{1} ; \ldots ; s_{k} \rightarrow t_{k}$ in $R$, we prepare $k$ fresh function symbols $U_{1}^{\rho}, \ldots, U_{k}^{\rho}$, called $U$ symbols, that do not appear in $\mathcal{F}$. We transform $\rho: l \rightarrow r \Leftarrow s_{1} \rightarrow$ $t_{1} ; \ldots ; s_{k} \rightarrow t_{k}$ into a set $\mathbb{U}(\rho)$ of $k+1$ unconditional rewrite rules as follows:

$$
\mathbb{U}(\rho)=\left\{\begin{aligned}
l & \rightarrow U_{1}^{\rho}\left(s_{1}, \overrightarrow{X_{1}}\right) \\
U_{1}^{\rho}\left(t_{1}, \overrightarrow{X_{1}}\right) & \rightarrow U_{2}^{\rho}\left(s_{2}, \overrightarrow{X_{2}}\right) \\
\vdots & \\
U_{k}^{\rho}\left(t_{k}, \overrightarrow{X_{k}}\right) & \rightarrow r
\end{aligned}\right\}
$$

where $X_{i}=\operatorname{Var}\left(l, t_{1}, \ldots, t_{i-1}\right)$ for all $1 \leq i \leq k$. Note that $\mathbb{U}\left(l^{\prime} \rightarrow r^{\prime}\right)=\left\{l^{\prime} \rightarrow r^{\prime}\right\}$. $\mathbb{U}$ is extended to eDCTRSs (i.e., $\left.\mathbb{U}(R)=\bigcup_{\rho \in R} \mathbb{U}(\rho)\right)$ and $\mathbb{U}(R)$ is an eTRS over the extended signature $\mathcal{F}_{\mathbb{U}(R)}=\mathcal{F} \cup\left\{U_{i}^{\rho} \mid \rho: l \rightarrow r \Leftarrow s_{1} \rightarrow t_{1} ; \ldots ; s_{k} \rightarrow t_{k} \in R, 1 \leq i \leq k\right\}$.

It is clear that $\rightarrow_{R} \subseteq \rightarrow_{\mathbb{U}(R)}^{*}$, and $\mathbb{U}\left(R^{\prime} \uplus R\right)=R^{\prime} \cup \mathbb{U}(R)$ if $R^{\prime}$ is unconditional. Moreover, by definition, $\mathbb{U}$ has compositionality, finiteness, and emptiness. Thus, $\mathbb{U}$ is a tidy unraveling for eDCTRSs.

The variant $\mathbb{U}_{\text {opt }}$ of Ohlebusch's unraveling $\mathbb{U}$ is proposed in both [18] and [7]. For a conditional rewrite rule $\rho: l \rightarrow r \Leftarrow s_{1} \rightarrow t_{1} ; \ldots ; s_{k} \rightarrow t_{k}$, the set $\mathbb{U}_{\text {opt }}(\rho)$ of unconditional rewrite rules is defined by replacing $\vec{X}_{i}$ in $\mathbb{U}(\rho)$ by $\left.\overrightarrow{X_{i} \cap \mathcal{V} a r\left(r, t_{i}, s_{i+1}, t_{i+1}, \ldots, s_{k}, t_{k}\right.}\right)$ for all $1 \leq i \leq k$ :

$$
\mathbb{U}_{\text {opt }}(\rho)=\left\{\begin{aligned}
l & \rightarrow U_{1}^{\rho}\left(s_{1}, \overrightarrow{X_{1} \cap Y_{1}}\right) \\
U_{1}^{\rho}\left(t_{1}, \overrightarrow{X_{1} \cap Y_{1}}\right) & \rightarrow U_{2}^{\rho}\left(s_{2}, \overline{X_{2} \cap Y_{2}}\right) \\
& \vdots \\
U_{k}^{\rho}\left(t_{k}, \overrightarrow{X_{k} \cap Y_{k}}\right) & \rightarrow r
\end{aligned}\right\}
$$

where $Y_{i}=\mathcal{V} \operatorname{Var}\left(r, t_{i}, s_{i+1}, t_{i+1}, \ldots, s_{k}, t_{k}\right)$ for all $1 \leq i \leq k$. Note that $\mathbb{U}_{\text {opt }}(R)$ is an eTRS over the extended signature $\mathcal{F}_{\mathbb{U}_{\mathrm{opt}}(R)}$ where $\mathcal{F}_{\mathbb{U}_{\mathrm{opt}}(R)}=\mathcal{F} \cup\left\{U_{i}^{\rho} \mid \rho: l \rightarrow r \Leftarrow s_{1} \rightarrow\right.$

\footnotetext{
${ }^{1}$ In the original definition [15], not the property $\rightarrow_{R} \subseteq \rightarrow_{U(R)}^{*}$ but the property $\downarrow_{R} \subseteq \downarrow_{U(R)}$ is imposed. Under this property, unravelings are not complete in general. For example, if $\rightarrow_{R} \subseteq \leftarrow_{U(R)}^{*}$, then $U$ is an unraveling. However, all the existing unravelings are designed so as to satisfy $\rightarrow_{R} \subseteq \rightarrow_{U(R)}^{*}$, that is implicitly required of unravelings. For this reason, this paper imposes the more restrictive property $\rightarrow_{R} \subseteq \rightarrow_{U(R)}^{*}$.
} 
$\left.t_{1} ; \ldots ; s_{k} \rightarrow t_{k} \in R, 1 \leq i \leq k\right\} 2$ Note also that $\mathbb{U}_{\text {opt }}$ is a tidy unraveling for eDCTRSs. $Y_{i}$ above, the set of variables appearing in any of $r, t_{i}, s_{i+1}, t_{i+1}, \ldots, s_{k}, t_{k}$, is the set of variables that are referred after $s_{i}$ is considered. Thus, $X_{i} \cap Y_{i}$ is the set of variables that appear in any of $l, t_{1}, \ldots, t_{i-1}$ and also appear after $s_{i}$ is considered, and hence $\overrightarrow{X_{i} \cap Y_{i}}$ is used for propagating only the variables that are referred later. On the other hand, $\vec{X}_{i}$ in Definition 3.1 is used for propagating all the appeared variables. This is the only difference between $\mathbb{U}$ and $\mathbb{U}_{\text {opt }}$, and the reason why $\mathbb{U}_{\text {opt }}$ is sometimes called an optimized variant of $\mathbb{U}$. Note that all of the following are equivalent:

- $R$ is of Type 3 ,

- $\mathbb{U}(R)$ has no extra variables, and

- $\mathbb{U}_{\text {opt }}(R)$ has no extra variables.

In the rest of the paper, unless noted otherwise, we use the label $\rho$ for presenting a conditional rewrite rule $l \rightarrow r \Leftarrow s_{1} \rightarrow t_{1} ; \ldots ; s_{k} \rightarrow t_{k}$, and we denote the sets $\operatorname{Var}\left(l, t_{1}, \ldots, t_{i-1}\right)$, $\mathcal{V} a r\left(r, t_{i}, s_{i+1}, t_{i+1}, \ldots, s_{k}, t_{k}\right)$, and $X_{i} \cap Y_{i}$ by $X_{i}, Y_{i}$, and $Z_{i}$, respectively.

Example 3.2. Consider the following TRS defining addition and multiplication of natural numbers encoded as $0, \mathrm{~s}(0), \mathrm{s}(\mathrm{s}(0)), \ldots$ :

$$
R_{1}=\left\{\begin{aligned}
\operatorname{add}(0, y) & \rightarrow y \\
\operatorname{add}(\mathrm{s}(x), y) & \rightarrow \mathrm{s}(\operatorname{add}(x, y)) \\
\operatorname{mult}(0, y) & \rightarrow 0 \\
\operatorname{mult}(x, 0) & \rightarrow 0 \\
\operatorname{mult}(\mathrm{s}(x), \mathrm{s}(y)) & \rightarrow \mathrm{s}(\operatorname{add}(\operatorname{mult}(x, \mathrm{~s}(y)), y))
\end{aligned}\right\}
$$

The inversion method in [18] inverts this TRS to the following DCTRS $R_{2}$ where add ${ }^{-1}$ and mult $^{-1}$ are function symbols that define the inverse relation of add and mult, respectively 3 and $\mathrm{tp}_{2}$ is a binary constructor for representing tuples of two terms:

$$
R_{2}=\left\{\begin{aligned}
\operatorname{add}^{-1}(y) & \rightarrow \operatorname{tp}_{2}(0, y) \\
\operatorname{add}^{-1}(\mathrm{~s}(z)) & \rightarrow \operatorname{tp}_{2}(\mathrm{~s}(x), y) \Leftarrow \operatorname{add}^{-1}(z) \rightarrow \operatorname{tp}_{2}(x, y) \\
\operatorname{mult}^{-1}(0) & \rightarrow \operatorname{tp}_{2}(0, y) \\
\operatorname{mult}^{-1}(0) & \rightarrow \operatorname{tp}_{2}(x, 0) \\
\operatorname{mult}^{-1}(\mathrm{~s}(z)) & \rightarrow \operatorname{tp}_{2}(\mathrm{~s}(x), \mathrm{s}(y)) \Leftarrow \operatorname{add}^{-1}(z) \rightarrow \operatorname{tp}_{2}(w, y) ; \operatorname{mult}^{-1}(w) \rightarrow \operatorname{tp}_{2}(x, \mathrm{~s}(y))
\end{aligned}\right\}
$$

This DCTRS is unraveled by $\mathbb{U}$ and $\mathbb{U}_{\text {opt }}$ as follows:

$$
\mathbb{U}\left(R_{2}\right)=\left\{\begin{aligned}
& \vdots \\
& \operatorname{add}^{-1}(\mathrm{~s}(z)) \rightarrow \mathrm{U}_{1}\left(\operatorname{add}^{-1}(z), z\right) \\
& \mathrm{U}_{1}\left(\operatorname{tp}_{2}(x, y), z\right) \rightarrow \operatorname{tp}_{2}(\mathrm{~s}(x), y) \\
& \vdots \\
& \operatorname{mult}^{-1}(\mathrm{~s}(z)) \rightarrow \mathrm{U}_{2}\left(\operatorname{add}^{-1}(z), z\right) \\
& \mathrm{U}_{2}\left(\operatorname{tp}_{2}(w, y), z\right) \rightarrow \mathrm{U}_{3}\left(\operatorname{mult}^{-1}(w), z, w, y\right) \\
& \mathrm{U}_{3}\left(\operatorname{tp}_{2}(x, \mathrm{~s}(y)), z, w, y\right) \rightarrow \operatorname{tp}_{2}(\mathrm{~s}(x), \mathrm{s}(y))
\end{aligned}\right\}
$$

\footnotetext{
2 The extended signatures $\mathcal{F}_{\mathbb{U}(R)}$ and $\mathcal{F}_{\mathbb{U}_{\text {opt }}(R)}$ are not equivalent in terms of the arities of U symbols (see, e.g., Example 3.2). We distinguish between these extended signatures since we deal with mappings from $\mathcal{T}\left(\mathcal{F}_{\mathbb{U}(R)}, \mathcal{V}\right)$ to $\mathcal{T}\left(\mathcal{F}_{\mathbb{U}_{\text {opt }}(R)}, \mathcal{V}\right)$ in Subsection 5.2

3 As inverse computation of $\operatorname{add}\left(\mathrm{s}^{m}(0), \mathrm{s}^{n}(0)\right) \rightarrow_{R_{1}}^{*} \mathrm{~s}^{m+n}(0)$ and $\operatorname{mult}\left(\mathrm{s}^{m}(0), \mathrm{s}^{n}(0)\right) \rightarrow{ }_{R_{1}}^{*} \mathrm{~s}^{m \times n}(0)$, we have the derivations $\operatorname{add}^{-1}\left(\mathrm{~s}^{m+n}(0)\right) \rightarrow_{R_{2}}^{*} \operatorname{tp}_{2}\left(\mathrm{~s}^{m}(0), \mathrm{s}^{n}(0)\right)$ and mult ${ }^{-1}\left(\mathrm{~s}^{m \times n}(0)\right) \rightarrow_{R_{2}}^{*} \operatorname{tp}_{2}\left(\mathrm{~s}^{m}(0), \mathrm{s}^{n}(0)\right)$.
} 


$$
\mathbb{U}_{\text {opt }}\left(R_{2}\right)=\left\{\begin{aligned}
\vdots \\
\operatorname{add}^{-1}(\mathrm{~s}(z)) \rightarrow \mathrm{U}_{1}\left(\mathrm{add}^{-1}(z)\right) \\
\mathrm{U}_{1}\left(\operatorname{tp}_{2}(x, y)\right) \rightarrow \operatorname{tp}_{2}(\mathrm{~s}(x), y) \\
\vdots \\
\operatorname{mult}^{-1}(\mathrm{~s}(z)) \rightarrow \mathrm{U}_{2}\left(\operatorname{add}^{-1}(z)\right) \\
\mathrm{U}_{2}\left(\operatorname{tp}_{2}(w, y)\right) \rightarrow \mathrm{U}_{3}\left(\operatorname{mult}^{-1}(w), y\right) \\
\mathrm{U}_{3}\left(\operatorname{tp}_{2}(x, \mathrm{~s}(y)), y\right) \rightarrow \operatorname{tp}_{2}(\mathrm{~s}(x), \mathrm{s}(y))
\end{aligned}\right\}
$$

Unravelings are not sound in general. The CTRS shown in the following example is a counterexample against soundness of both $\mathbb{U}$ and $\mathbb{U}_{\text {opt }}$.

Example 3.3 ([15, 26]). Consider the following 3-DCTRS $R_{3}$ and its unraveled TRSs:

$$
\begin{gathered}
R_{3}=\left\{\begin{array}{c}
\mathrm{f}(x) \rightarrow x \Leftarrow x \rightarrow \mathrm{e} \\
\mathrm{g}(\mathrm{d}, x, x) \rightarrow \mathrm{A} \\
\mathrm{h}(x, x) \rightarrow \mathrm{g}(x, x, \mathrm{f}(\mathrm{k}))
\end{array}\right\} \cup R_{0} \\
\mathbb{U}\left(R_{3}\right)=\mathbb{U}_{\text {opt }}\left(R_{3}\right)=\left\{\begin{array}{c}
\mathrm{f}(x) \rightarrow \mathrm{U}_{4}(x, x) \\
\mathrm{U}_{4}(\mathrm{e}, x) \rightarrow x \\
\vdots
\end{array}\right\} \cup R_{0}
\end{gathered}
$$

where

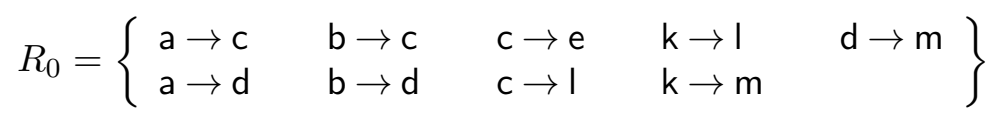

We have a reduction sequence of $\mathbb{U}_{\text {opt }}\left(R_{3}\right)$ from $\mathrm{h}(\mathrm{f}(\mathrm{a}), \mathrm{f}(\mathrm{b}))$ to $\mathrm{A}$ :

$$
\begin{aligned}
\mathrm{h}(\mathrm{f}(\mathrm{a}), \mathrm{f}(\mathrm{b})) & \rightarrow \mathbb{U}_{\text {opt }}^{*}\left(R_{3}\right) \mathrm{h}\left(\mathrm{U}_{4}(\mathrm{c}, \mathrm{d}), \mathrm{U}_{4}(\mathrm{c}, \mathrm{d})\right) \rightarrow \mathbb{U}_{\mathrm{opt}}\left(R_{3}\right) \mathrm{g}\left(\mathrm{U}_{4}(\mathrm{c}, \mathrm{d}), \mathrm{U}_{4}(\mathrm{c}, \mathrm{d}), \mathrm{f}(\mathrm{k})\right) \\
& \rightarrow_{\mathbb{U}_{\text {opt }}\left(R_{3}\right)}^{*} \mathrm{~g}\left(\mathrm{~d}, \mathrm{U}_{4}(\mathrm{l}, \mathrm{m}), \mathrm{U}_{4}(\mathrm{l}, \mathrm{m})\right) \rightarrow_{\mathbb{U}_{\mathrm{opt}}\left(R_{3}\right)} \mathrm{A}
\end{aligned}
$$

However, we have no similar reduction sequence of $R_{3}$, i.e., $\mathrm{h}(\mathrm{f}(\mathrm{a}), \mathrm{f}(\mathrm{b})) t_{R_{3}}^{*} \mathrm{~A}$. Thus, neither $\mathbb{U}$ nor $\mathbb{U}_{\text {opt }}$ is sound for $R_{3}$. Note that being (ultra-)overlapping-systems is not sufficient for soundness of $\mathbb{U}_{\text {opt }}$ and $\mathbb{U}$ since $\mathbb{U}_{\text {opt }}\left(R_{3}\right)\left(=\mathbb{U}\left(R_{3}\right)\right)$ is an overlapping system.

Soundness of $\mathbb{U}$ can be recovered by restricting the reduction of the unraveled TRSs to the context-sensitive reduction [13] with the replacement mapping determined by means of the application of $\mathbb{U}[28,29]$ : $\mathbb{U}$ is sound for a 3-DCTRS $R$ if the reduction of $\mathbb{U}(R)$ is restricted to context-sensitive rewriting with the replacement mapping $\mu$ such that $\mu\left(U_{i}^{\rho}\right)$ $=\{1\}$ for any $\mathrm{U}$ symbol $U_{i}^{\rho}$ - the replacement mapping forbids reducing any redex inside the second or later arguments of $U$ symbols. This holds for $\mathbb{U}_{\text {opt }}$ by restricting the contextsensitive reduction to the reduction with the membership condition [34], a very complicated restriction that soundness of $\mathbb{U}$ does not require. In this respect, $\mathbb{U}_{\text {opt }}$ does not look like an "optimized" variant of $\mathbb{U}$. The following examples show that neither the context-sensitive nor membership conditions above is sufficient on its own for soundness of $\mathbb{U}_{\text {opt }}$.

Example 3.4. Consider the following DCTRS and its unraveled TRSs:

$$
\begin{aligned}
& R_{4}=\{\mathrm{f}(x, y) \rightarrow x \Leftarrow \mathrm{g}(x) \rightarrow z ; \mathrm{g}(y) \rightarrow z \quad \mathrm{~g}(x) \rightarrow \mathrm{c} \Leftarrow \mathrm{d} \rightarrow \mathrm{c}\} \\
& \mathbb{U}\left(R_{4}\right)=\left\{\begin{aligned}
\mathrm{f}(x, y) & \rightarrow \mathrm{U}_{5}(\mathrm{~g}(x), x, y) & \mathrm{U}_{5}(z, x, y) & \rightarrow \mathrm{U}_{6}(\mathrm{~g}(y), x, y, z) \\
\mathrm{g}(x) & \rightarrow \mathrm{U}_{7}(\mathrm{~d}, x) & \mathrm{U}_{7}(\mathrm{c}, x) \rightarrow \mathrm{c} &
\end{aligned}\right\} \\
& \mathbb{U}_{\text {opt }}\left(R_{4}\right)=\left\{\begin{aligned}
\mathrm{f}(x, y) & \rightarrow \mathrm{U}_{5}(\mathrm{~g}(x), x, y) & \mathrm{U}_{5}(z, x, y) & \rightarrow \mathrm{U}_{6}(\mathrm{~g}(y), x, z) \\
\mathrm{g}(x) & \rightarrow \mathrm{U}_{7}(\mathrm{~d}) & \mathrm{U}_{7}(\mathrm{c}) & \rightarrow \mathrm{c}
\end{aligned}\right.
\end{aligned}
$$


For the context-sensitive condition mentioned above, we forbid reducing any redex inside the second or third arguments of $U_{5}$ and $U_{6}$. We have the derivation $\mathrm{f}(\mathrm{a}, \mathrm{b}) \rightarrow_{\mathbb{U}_{\text {opt }}\left(R_{4}\right)}^{*}$ $\mathrm{U}_{6}\left(\mathrm{U}_{7}(\mathrm{~d}), \mathrm{a}, \mathrm{U}_{7}(\mathrm{~d})\right) \rightarrow_{\mathbb{U}_{\mathrm{opt}}\left(R_{4}\right)}$ a under the context-sensitive condition, but this derivation is not possible in $R_{4}$. Therefore, the context-sensitive condition is not sufficient on its own for soundness of $\mathbb{U}_{\text {opt }}$. Note that the derivation $\mathrm{f}(\mathrm{a}, \mathrm{b}) \rightarrow^{*}$ a does not hold in $\mathbb{U}\left(R_{4}\right)$ under the context-sensitive condition, either, since $f(a, b)$ can be reduced to both $U_{6}\left(U_{7}(d, b), a, g(a)\right)$ and $\mathrm{U}_{6}\left(\mathrm{U}_{7}(\mathrm{~d}, \mathrm{a}), \mathrm{a}, \mathrm{U}_{7}(\mathrm{~d}, \mathrm{~b})\right)$, but they are not reduced any more.

Example 3.5. Consider the following DCTRS $R_{5}$ and its unraveled TRSs:

$$
\begin{aligned}
& R_{5}=\{\mathrm{f}(x) \rightarrow x \Leftarrow x \rightarrow \mathrm{a} ; \mathrm{b} \rightarrow x \quad \mathrm{a} \rightarrow \mathrm{b}\} \\
& \mathbb{U}\left(R_{5}\right)=\mathbb{U}_{\text {opt }}\left(R_{5}\right)=\left\{\mathrm{f}(x) \rightarrow \mathrm{U}_{8}(x, x) \quad \mathrm{U}_{8}(\mathrm{a}, x) \rightarrow \mathrm{U}_{9}(\mathrm{~b}, x) \quad \mathrm{U}_{9}(x, x) \rightarrow x \quad \ldots\right\}
\end{aligned}
$$

For the membership condition, we forbid reducing any redex that has a proper subterm containing U symbols. We have the derivation $\mathrm{f}(\mathrm{a}) \rightarrow_{\mathbb{U}_{\text {opt }}\left(R_{5}\right)}^{*} \mathrm{U}_{9}(\mathrm{~b}, \mathrm{a}) \rightarrow_{\mathbb{U}_{\mathrm{opt}}\left(R_{5}\right)} \mathrm{U}_{9}(\mathrm{~b}, \mathrm{~b})$ $\rightarrow_{\mathbb{U}_{\mathrm{opt}}\left(R_{5}\right)}$ b under the membership condition, but this derivation is not possible in $R_{5}$. Therefore, the membership condition is not sufficient on its own for soundness of either $\mathbb{U}_{\text {opt }}$ or $\mathbb{U}$.

To analyze syntactic relationships between eDCTRS and the corresponding unraveled eTRSs, we recall ultra-properties of DCTRSs [15, 16], extending them to eDCTRSs.

Definition 3.6 (ultra-property [15, 16]). Let $P$ be a property on (extended) conditional rewrite rules, and $U$ be an unraveling. An (extended) conditional rewrite rule $\rho$ is said to be ultra- $P$ w.r.t. $U(U-P)$ if all the rules in $U(\rho)$ satisfy the property $P$. An eDCTRS $R$ is said to be ultra-P w.r.t. $U(U-P)$ if all the rules in $R$ are $U-P$.

Example 3.7. The DCTRS $R_{2}$ in Example 3.2 is non-LV and non-RV w.r.t. both $\mathbb{U}$ and $\mathbb{U}_{\text {opt }}$, but $R_{2}$ is not $\mathbb{U}-\mathrm{LL}, \mathbb{U}-\mathrm{RL}$, or $\mathbb{U}-\mathrm{NE}$ either, while $R_{2}$ is $\mathbb{U}_{\text {opt }}-\mathrm{RL}$ and $\mathbb{U}_{\text {opt }}-\mathrm{NE}$, but not $\mathbb{U}_{\text {opt }}-\mathrm{LL}$.

Note that the $\mathbb{U}_{\text {opt }}$-LL property is the same as semi-linearity in [16. Roughly speaking, the conditional parts of $\mathbb{U}_{\mathrm{opt}}-\mathrm{LL}$ conditional rules correspond to the let structures of functional programs.

The $\mathbb{U}_{\text {opt }}-\mathrm{LL}, \mathbb{U}_{\mathrm{opt}}-\mathrm{RL}$, and $\mathbb{U}_{\mathrm{opt}}-\mathrm{NE}$ properties of conditional rewrite rules are identical with the following syntactic properties of DCTRSs, respectively.

Theorem 3.8. Let $\rho: l \rightarrow r \Leftarrow s_{1} \rightarrow t_{1} ; \ldots ; s_{k} \rightarrow t_{k}$ be an extended deterministic conditional rewrite rule. Then, all of the following hold:

(1) $\rho$ is $\mathbb{U}_{\text {opt }}-L L$ iff all of $l, t_{1}, \ldots, t_{k}$ are linear and $\mathcal{V} a r\left(t_{i}\right) \cap X_{i}=\emptyset$ for all $1 \leq i \leq k$,

(2) $\rho$ is $\mathbb{U}_{\mathrm{opt}}-R L$ iff all of $r, s_{1}, \ldots, s_{k}$ are linear and $\mathcal{V}$ ar $\left(s_{i}\right) \cap Y_{i}=\emptyset$ for all $1 \leq i \leq k$, and

(3) $\rho$ is $\mathbb{U}_{\mathrm{opt}}-N E$ iff $\mathcal{V} a r(l) \subseteq \mathcal{V} a r\left(r, s_{1}, \ldots, s_{k}\right)$ and $\mathcal{V} a r\left(t_{i}\right) \subseteq \mathcal{V} a r\left(r, s_{i+1}, \ldots, s_{k}\right)$ for all $1 \leq i \leq k$.

Proof. The proof can be seen in Appendix A.1. 
The sufficient and necessary condition for the $\mathbb{U}_{\text {opt }}-\mathrm{NE}$ property in Theorem 3.8 is equivalent to the one shown in [18, 23] since the following are equivalent:

- $\mathcal{V} a r(l) \subseteq \mathcal{V} a r\left(r, s_{1}, t_{1}, \ldots, s_{k}, t_{k}\right)$ and $\mathcal{V} a r\left(t_{i}\right) \subseteq \mathcal{V} a r\left(r, s_{i+1}, t_{i+1}, \ldots, s_{k}, t_{k}\right)$ for all $1 \leq i$ $\leq k$, and

- $\mathcal{V} a r(l) \subseteq \mathcal{V} a r\left(r, s_{1}, \ldots, s_{k}\right)$ and $\mathcal{V} a r\left(t_{i}\right) \subseteq \mathcal{V} a r\left(r, s_{i+1}, \ldots, s_{k}\right)$ for all $1 \leq i \leq k$.

Neither the second nor third claims in Theorem 3.8 holds for $\mathbb{U}$ ( $c f$., Examples 3.2, 3.7), while the first one holds for $\mathbb{U}$. Quite restricted variants of the second and third claims hold for $\mathbb{U}$.

Theorem 3.9. Let $\rho: l \rightarrow r \Leftarrow s_{1} \rightarrow t_{1} ; \ldots ; s_{k} \rightarrow t_{k}$ be an extended deterministic conditional rewrite rule. Then, all of the following hold:

(1) $\rho$ is $\mathbb{U}-L L$ iff all of $l, t_{1}, \ldots, t_{k}$ are linear and $\mathcal{V}$ ar $\left(t_{i}\right) \cap X_{i}=\emptyset$ for all $1 \leq i \leq k$,

(2) $\rho$ is $\mathbb{U}-R L$ iff $r$ is linear and all of $s_{1}, \ldots, s_{k}$ are ground, and

(3) $\rho$ is $\mathbb{U}-N E$ iff $\mathcal{V} a r\left(l, t_{1}, \ldots, t_{k}\right) \subseteq \mathcal{V}$ ar $(r)$.

Proof. The proof can be seen in Appendix A.2.

Note that the $\mathbb{U}-L L$ and $\mathbb{U}_{\text {opt }}-$ LL properties are equivalent. Theorems 3.8 , 3.9 lead to the following relationship between the ultra-RL and ultra-NE properties w.r.t. $\mathbb{U}$ and $\mathbb{U}_{\text {opt }}$.

Corollary 3.10. The $\mathbb{U}-R L$ and $\mathbb{U}-N E$ properties imply the $\mathbb{U}_{\mathrm{opt}}-R L$ and $\mathbb{U}_{\mathrm{opt}}-N E$ properties, respectively.

As for the non-LV and non-RV properties, we have the following relationships between eDCTRSs and the corresponding unraveled eTRSs.

Proposition 3.11. Let $U$ be either $\mathbb{U}$ or $\mathbb{U}_{\mathrm{opt}}, R$ be an eDCTRS, and $\rho$ be an (extended) conditional rewrite rule. Then, all of the following hold:

- $\rho$ is non-LV iff so is $U(\rho)$,

- $\rho$ is non- $R V$ iff so is $U(\rho)$,

- $R$ is non- $L V$ iff so is $U(R)$, and

- $R$ is non- $R V$ iff so is $U(R)$.

Proof. Trivial by definition.

We recognize from Proposition 3.11 that, for both $\mathbb{U}$ and $\mathbb{U}_{\text {opt }}$, the non-LV and non-RV properties are equivalent to the ultra-non-LV and ultra-non-RV properties, respectively.

\section{Soundness of the Optimized UnRaveling}

In this section, we first show that the optimized unraveling $\mathbb{U}_{\text {opt }}$ is sound for $\mathbb{U}_{\text {opt }}-\mathrm{LL} 3$ DCTRSs. Then, we show that $\mathbb{U}_{\text {opt }}$ is sound for DCTRSs that are both $\mathbb{U}_{\text {opt }}-\mathrm{RL}$ and $\mathbb{U}_{\text {opt }}-N E$. Finally, we extend the result on soundness for $\mathbb{U}_{\text {opt }}-L L 3$-DCTRSs to $\mathbb{U}_{\text {opt }}-\mathrm{LL}$ DCTRSs, i.e., $\mathbb{U}_{\mathrm{opt}}$ is sound for a $\mathbb{U}_{\mathrm{opt}}-\mathrm{LL}$ DCTRS if the reduction of the corresponding unraveled EV-TRS is restricted to EV-safe ones (see Definition 4.14). In the rest of this paper, we write the terminology "RLNE" for "RL and NE". 
4.1. Soundness on Ultra-Left-Linearity. In this subsection, we first show that the LL property is not a soundness condition of either $\mathbb{U}_{\text {opt }}$ or $\mathbb{U}$, and then we show that $\mathbb{U}_{\text {opt }}$ is sound for $\mathbb{U}_{\text {opt }}$-LL 3-DCTRSs. This result also holds for arbitrary DCTRSs under some restriction to reduction. Although we first show the case of 3-DCTRSs to make the essential scheme of the proof clear, we will extend the result in this subsection to DCTRSs in Subsection 4.4,

As described in Section 1, the LL property is a soundness condition for unravelings for normal CTRSs. In contrast, the LL property is not a soundness condition for either $\mathbb{U}_{\text {opt }}$ or $\mathbb{U}$.

Example 4.1. Consider the following DCTRS obtained from $R_{3}$ by left-linearizing:

$$
R_{6}=\left\{\begin{array}{c}
\mathrm{f}(x) \rightarrow x \Leftarrow x \rightarrow \mathrm{e} \\
\mathrm{g}(\mathrm{d}, x, y) \rightarrow \mathrm{A} \Leftarrow y \rightarrow x \\
\mathrm{~h}(x, y) \rightarrow \mathrm{g}(x, y, \mathrm{f}(\mathrm{k})) \Leftarrow y \rightarrow x
\end{array}\right\} \cup R_{0}
$$

$R_{6}$ is unraveled by $\mathbb{U}_{\text {opt }}$ and $\mathbb{U}$ to the following TRSs:

$$
\begin{aligned}
& \mathbb{U}_{\text {opt }}\left(R_{6}\right)=\left\{\begin{aligned}
\mathrm{f}(x) & \rightarrow \mathrm{U}_{10}(x, x) & \mathrm{U}_{10}(\mathrm{e}, x) & \rightarrow x \\
\mathrm{~g}(\mathrm{~d}, x, y) & \rightarrow \mathrm{U}_{11}(y, x) & \mathrm{U}_{11}(x, x) & \rightarrow \mathrm{A} \\
\mathrm{h}(x, y) & \rightarrow \mathrm{U}_{12}(y, x, y) & \mathrm{U}_{12}(x, x, y) & \rightarrow \mathrm{g}(x, y, \mathrm{f}(\mathrm{k}))
\end{aligned}\right\} \cup R_{0} \\
& \mathbb{U}\left(R_{6}\right)=\left\{\begin{aligned}
\mathrm{f}(x) & \rightarrow \mathrm{U}_{10}(x, x) & \mathrm{U}_{10}(\mathrm{e}, x) & \rightarrow x \\
\mathrm{~g}(\mathrm{~d}, x, y) & \rightarrow \mathrm{U}_{11}(y, x, y) & \mathrm{U}_{11}(x, x, y) & \rightarrow \mathrm{A} \\
\mathrm{h}(x, y) & \rightarrow \mathrm{U}_{12}(y, x, y) & \mathrm{U}_{12}(x, x, y) & \rightarrow \mathrm{g}(x, y, \mathrm{f}(\mathrm{k}))
\end{aligned}\right\} \cup R_{0}
\end{aligned}
$$

As in Example 3.3, we have the derivations $\mathrm{h}(\mathrm{f}(\mathrm{a}), \mathrm{f}(\mathrm{b})) \rightarrow_{\mathbb{U}_{\text {opt }}\left(R_{6}\right)}^{*} \mathrm{~A}$ and $\mathrm{h}(\mathrm{f}(\mathrm{a}), \mathrm{f}(\mathrm{b})) \rightarrow_{\mathbb{U}\left(R_{6}\right)}^{*}$ A, but $\mathrm{h}(\mathrm{f}(\mathrm{a}), \mathrm{f}(\mathrm{b}))$ cannot be reduced by $R_{6}$ to $A$. Therefore, neither $\mathbb{U}_{\text {opt }}$ nor $\mathbb{U}$ is sound for $R_{6}$.

The LL property of normal CTRSs is equivalent to the $\mathbb{U}_{\text {opt }}-\mathrm{LL}$ property since the righthand sides $n_{i}$ of conditions $s_{i} \rightarrow n_{i}$ are ground. In contrast, the LL property of DCTRSs is not equivalent to the ultra-LL property in general (see $\mathbb{U}_{\text {opt }}\left(R_{6}\right)$ and $\mathbb{U}\left(R_{6}\right)$ in Example 4.1). Moreover, the LL property of the unraveled TRSs plays an important role in the existing proof of soundness. Thus, the ultra-LL property seems a soundness condition for $\mathbb{U}_{\text {opt }}$ (and also for $\mathbb{U})$.

The soundness result of this subsection is a consequence of the following key lemma: given a derivation $s \rightarrow_{\mathbb{U}_{\text {opt }}(R)}^{*} t \sigma$ with $s, t \in \mathcal{T}(\mathcal{F}, \mathcal{V})$, the lemma guarantees the existence of an intermediate term $t \theta \in \mathcal{T}(\mathcal{F}, \mathcal{V})$ such that $s \rightarrow_{R}^{*} t \theta \rightarrow_{\mathbb{U}_{\mathrm{opt}}(R)}^{*} t \sigma$ and, moreover, $t \theta=$ $t \sigma$ whenever $t \sigma \in \mathcal{T}(\mathcal{F}, \mathcal{V})$.

Lemma 4.2. Let $R$ be a $\mathbb{U}_{\text {opt }}-L L$ 3-eDCTRS over a signature $\mathcal{F}$, s be a term in $\mathcal{T}(\mathcal{F}, \mathcal{V})$, $t$ be a linear term in $\mathcal{T}(\mathcal{F}, \mathcal{V})$, and $\sigma$ be a substitution in $\mathcal{S} u b\left(\mathcal{F}_{\mathbb{U}_{\mathrm{opt}}(R)}, \mathcal{V}\right)$. Suppose that $R$ is non- $L V$ or non- $R V$. If $s \rightrightarrows_{\mathbb{U}_{\mathrm{opt}}(R)}^{n}$ t $\sigma$ for some $n \geq 0$, then there exists a substitution $\theta$ in $\mathcal{S} u b(\mathcal{F}, \mathcal{V})$ such that

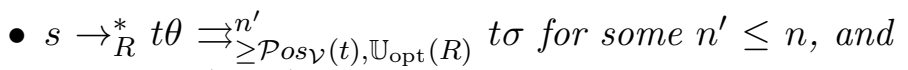

- if $t \sigma \in \mathcal{T}(\mathcal{F}, \mathcal{V})$, then $t \theta=t \sigma$.

Proof. The proof can be seen in Appendix A.3. 
As a consequence of Lemma 4.2, we show the main theorem of this subsection.

Theorem 4.3. $\mathbb{U}_{\mathrm{opt}}$ is sound for $\mathbb{U}_{\mathrm{opt}}-L L$ 3-eDCTRSs that are non-LV or non-RV.

Proof. Let $R$ be a 3-eDCTRS over a signature $\mathcal{F}$, that is non-LV or non-RV. Suppose that $s \rightarrow_{\mathbb{U}_{\text {opt }}(R)}^{*} t$ and $s, t \in \mathcal{T}(\mathcal{F}, \mathcal{V})$. Since a single step of $\rightarrow_{\mathbb{U}_{\mathrm{opt}}(R)}$ can be considered a single step of the parallel reduction, we have the derivation $s \rightrightarrows_{\mathbb{U}_{\text {opt }}(R)}^{*} t$. Let $x$ be a variable and $\sigma$ be a substitution such that $x \sigma=t$. Then, it follows from Lemma 4.2 that $s \rightarrow_{R}^{*} x \sigma=t$.

Example 4.4. Consider the following $\mathbb{U}_{\text {opt }}-\mathrm{LL}$ and non-LV DCTRS to define a splitting function split for lists of non-negative integers encoded as $0, \mathrm{~s}(0), \ldots$, e.g., split $(3,[2,5,1,4,3])$ $=([2,1],[5,4,3])$ :

$$
\begin{aligned}
R_{7}= & \left.\begin{array}{rl}
\operatorname{split}(x, \text { nil }) & \rightarrow \operatorname{tp}_{2}(\text { nil, nil }) \\
\operatorname{split}(x, \operatorname{cons}(y, y s)) & \rightarrow \operatorname{tp}_{2}\left(z s_{1}, \operatorname{cons}\left(y, z s_{2}\right)\right) \Leftarrow \operatorname{split}(x, y s) \rightarrow \operatorname{tp}_{2}\left(z s_{1}, z s_{2}\right) ; \text { le }(x, y) \rightarrow \text { true } \\
\operatorname{split}(x, \operatorname{cons}(y, y s)) & \rightarrow \operatorname{tp}_{2}\left(\operatorname{cons}\left(y, z s_{1}\right), z s_{2}\right) \Leftarrow \operatorname{split}(x, y s) \rightarrow \operatorname{tp}_{2}\left(z s_{1}, z s_{2}\right) ; \text { le }(x, y) \rightarrow \text { false } \\
\text { le }(0, y) & \rightarrow \text { true } \\
\text { le }(\mathrm{s}(x), 0) & \rightarrow \text { false } \\
\text { le }(x), \mathrm{s}(y)) & \rightarrow \text { le }(x, y)
\end{array}\right\}
\end{aligned}
$$

$R_{7}$ is unraveled by $\mathbb{U}_{\text {opt }}$ and $\mathbb{U}$ into the following TRSs:

$$
\mathbb{U}_{\text {opt }}\left(R_{7}\right)=\left\{\begin{aligned}
& \vdots \\
& \operatorname{split}(x, \operatorname{cons}(y, y s)) \rightarrow \mathrm{U}_{13}(\operatorname{split}(x, y s), x, y) \\
& \mathrm{U}_{13}\left(\operatorname{tp}_{2}\left(z s_{1}, z s_{2}\right), x, y\right) \rightarrow \mathrm{U}_{14}\left(\operatorname{le}(x, y), y, z s_{1}, z s_{2}\right) \\
& \mathrm{U}_{14}\left(\operatorname{true}, y, z s_{1}, z s_{2}\right) \rightarrow \operatorname{tp}_{2}\left(z s_{1}, \operatorname{cons}\left(y, z s_{2}\right)\right) \\
& \operatorname{split}(x, \operatorname{cons}(y, y s)) \rightarrow \mathrm{U}_{15}(\operatorname{split}(x, y s), x, y) \\
& \mathrm{U}_{15}\left(\operatorname{tp}_{2}\left(z s_{1}, z s_{2}\right), x, y\right) \rightarrow \mathrm{U}_{16}\left(\operatorname{le}(x, y), y, z s_{1}, z s_{2}\right) \\
& \mathrm{U}_{16}\left(\text { false }, y, z s_{1}, z s_{2}\right) \rightarrow \operatorname{tp}_{2}\left(\operatorname{cons}\left(y, z s_{1}\right), z s_{2}\right) \\
& \vdots
\end{aligned}\right\}
$$

We recognize from Theorem 4.3 that $\mathbb{U}_{\text {opt }}$ is sound for $R_{7}$.

Due to the technical proof of Lemma 4.2, we assumed that eDCTRSs are non-LV or non-RV. It is not known yet whether this assumption can be relaxed (removed) or not. However, this assumption is not so restrictive since every DCTRS is non-LV. Theorem 4.3 is not a direct consequence of the result in [16] on soundness for $\mathbb{U}_{\text {opt }}$-LL 3-DCTRSs since $\mathrm{U}$ symbols introduced by $\mathbb{U}_{\text {opt }}$ have less arguments than those introduced by the unraveling in [16].

4.2. Observing Unsoundness of Marchiori's Counterexample to Soundness. In the previous subsection, we conjectured and proved that the ultra-LL property is a soundness condition of $\mathbb{U}_{\text {opt }}$ since the property is already known to be a soundness condition of Marchiori's unraveling for normal CTRSs. To find other soundness conditions, in this subsection, we take a close look at the derivation $\mathrm{h}(\mathrm{f}(\mathrm{a}), \mathrm{f}(\mathrm{b})) \rightarrow_{\mathbb{U}_{\text {opt }}\left(R_{3}\right)}^{*} \mathrm{~A}$ in Example 3.3, observing the reason why $\mathbb{U}_{\text {opt }}$ is not sound for $R_{3}$ in Example 3.3 ,

Recall the derivation $\mathrm{h}(\mathrm{f}(\mathrm{a}), \mathrm{f}(\mathrm{b})) \rightarrow_{\mathbb{U}_{\mathrm{opt}}\left(R_{3}\right)}^{*} \mathrm{~A}$ in Example 3.3

$$
\begin{aligned}
& \mathrm{h}(\mathrm{f}(\mathrm{a}), \mathrm{f}(\mathrm{b})) \rightarrow_{\mathbb{U}_{\text {opt }}\left(R_{3}\right)}^{*} \mathrm{~h}\left(\mathrm{U}_{4}(\mathrm{c}, \mathrm{d}), \mathrm{U}_{4}(\mathrm{c}, \mathrm{d})\right) \rightarrow_{\mathbb{U}_{\mathrm{opt}}\left(R_{3}\right)} \mathrm{g}\left(\mathrm{U}_{4}(\mathrm{c}, \mathrm{d}), \mathrm{U}_{4}(\mathrm{c}, \mathrm{d}), \mathrm{f}(\mathrm{k})\right) \\
& \rightarrow_{\mathbb{U}_{\text {opt }}\left(R_{3}\right)}^{*} \mathrm{~g}\left(\mathrm{~d}, \mathrm{U}_{4}(\mathrm{I}, \mathrm{m}), \mathrm{U}_{4}(\mathrm{I}, \mathrm{m})\right) \rightarrow_{\mathbb{U}_{\mathrm{opt}}\left(R_{3}\right)} \mathrm{A}
\end{aligned}
$$


To succeed in this derivation, the following subderivations are necessary:

- to apply the rule $\mathrm{g}(\mathrm{d}, x, x) \rightarrow \mathrm{A}$, the subterm $\mathrm{f}(\mathrm{a})$ in the initial term is reduced to $\mathrm{d}$,

- to apply the rule $\mathrm{h}(x, x) \rightarrow \mathrm{g}(x, x, \mathrm{f}(\mathrm{k}))$, both the subterms $\mathrm{f}(\mathrm{a})$ and $\mathrm{f}(\mathrm{b})$ in the initial term are reduced to the same term, and

- to apply the rule $\mathrm{g}(\mathrm{d}, x, x) \rightarrow \mathrm{A}$, both the subterm $\mathrm{f}(\mathrm{b})$ in the initial term and the term $\mathrm{f}(\mathrm{k})$ derived from the application of $\mathrm{h}(x, x) \rightarrow \mathrm{g}(x, x, \mathrm{f}(\mathrm{k}))$ are reduced to the same term.

As a consequence, all of the terms $f(a), f(b)$, and $f(k)$ have to be reduced to the same term $\mathrm{d}$. However, this is impossible on the reduction of $R_{3}$. Nevertheless, in the above derivation, $\mathrm{h}(x, x) \rightarrow \mathrm{g}(x, x, \mathrm{f}(\mathrm{k}))$ is applied after reducing $\mathrm{f}(\mathrm{a})$ and $\mathrm{f}(\mathrm{b})$ to $\mathrm{U}_{4}(\mathrm{c}, \mathrm{d})$ : the $\mathrm{U}_{4}(\mathrm{c}, \mathrm{d})$, that derives from $f(a)$, is reduced to $d$, and the other $U_{4}(c, d)$, that derives from $f(b)$, is reduced to $\mathrm{U}_{4}(\mathrm{l}, \mathrm{m})$ in order to be the same as $\mathrm{f}(\mathrm{k})$. Finally, $\mathrm{g}(\mathrm{d}, x, x) \rightarrow \mathrm{A}$ is applied. These undesired subderivations must be caused by the non-RL rule $\mathrm{h}(x, x) \rightarrow \mathrm{g}(x, x, \mathrm{f}(\mathrm{k}))$ and the erasing rule $\mathrm{g}(\mathrm{d}, x, x) \rightarrow \mathrm{A}$ in $\mathbb{U}_{\text {opt }}\left(R_{3}\right)$. This stems from the following aspect:

- the application of $\mathrm{h}(x, x) \rightarrow \mathrm{g}(x, x, \mathrm{f}(\mathrm{k}))$ to $\mathrm{h}\left(\mathrm{U}_{4}(\mathrm{c}, \mathrm{d}), \mathrm{U}_{4}(\mathrm{c}, \mathrm{d})\right)$ keeps two occurrences of $\mathrm{U}_{4}(\mathrm{c}, \mathrm{d})$ that are intermediate states of evaluating $\mathrm{f}(\mathrm{a})$ and $\mathrm{f}(\mathrm{b})$, respectively, and each of occurrence has a capability to be reduced to a different term later although they should be the same, and

- $\mathrm{g}(\mathrm{d}, x, x) \rightarrow \mathrm{A}$ erases the two occurrences of $\mathrm{U}_{4}(\mathrm{l}, \mathrm{m})$ as if they derive from the same term (in fact, they derive from the terms $f(b)$ and $f(k)$, respectively, although $f(b)$ and $f(k)$ should be reduced to different terms).

Viewed in this light, we conjecture that the RLNE property of the unraveled TRSs is a sufficient condition for soundness of $\mathbb{U}_{\text {opt }}$. Note that the above issue does not arise in the case of ultra-LL DCTRSs since the LL property does not require equivalence at all between subterms in redexes.

In the next subsection, we will prove the conjecture above, by reducing soundness for a $\mathbb{U}_{\text {opt }}$-RLNE DCTRS to that for a DCTRS obtained by simply inverting. The key feature is that if a DCTRS is $\mathbb{U}_{\text {opt }}-\mathrm{NE}$, then,

- the unraveled TRS of the inverted one is equivalent to the inverted unraveled TRS of the DCTRS, and

- the inverted one is $\mathbb{U}_{\text {opt }}-\mathrm{LL}$ iff the DCTRS is $\mathbb{U}_{\text {opt }}-\mathrm{RL}$.

The converse of this approach is impossible since the first property above needs the $\mathbb{U}_{\text {opt }}-\mathrm{NE}$ property and not all $\mathbb{U}_{\text {opt }}-\mathrm{LL}$ DCTRSs have the $\mathbb{U}_{\text {opt }}-\mathrm{NE}$ property.

4.3. Soundness on Ultra-RLNE Property. In this subsection, we show that the optimized unraveling $\mathbb{U}_{\text {opt }}$ is sound for $\mathbb{U}_{\text {opt }}$-RLNE DCTRSs. To prove it, we reduce the soundness to that of $\mathbb{U}_{\text {opt }}$ for ultra-LL DCTRSs. Moreover, we provide examples showing that neither $\mathbb{U}_{\mathrm{opt}}-\mathrm{RL}$ nor $\mathbb{U}_{\mathrm{opt}}-\mathrm{NE}$ properties is sufficient on its own for soundness of $\mathbb{U}_{\text {opt }}$.

We first define the operation to transform eDCTRSs into eDCTRSs that define the inverse relation of the former eDCTRSs. Note that the "inverse" here is slightly distinct from "inverse" in the sense of program inversion.

Definition 4.5. Let $\rho: l \rightarrow r \Leftarrow s_{1} \rightarrow t_{1} ; \ldots ; s_{k} \rightarrow t_{k}$ be an (extended) conditional rewrite rule. We define the operation $(\cdot)^{-1}$ as follows:

$$
\left(l \rightarrow r \Leftarrow s_{1} \rightarrow t_{1} ; \ldots ; s_{k} \rightarrow t_{k}\right)^{-1}=r \rightarrow l \Leftarrow t_{k} \rightarrow s_{k} ; \ldots ; t_{1} \rightarrow s_{1} .
$$

This operation is extended to eDCTRSs as $(R)^{-1}=\left\{(\rho)^{-1} \mid \rho \in R\right\}$. 
For an eCTRS $R$, the inverse relation of $\rightarrow_{R}$ is equivalent to the reduction of $(R)^{-1}$.

Proposition 4.6. Let $R$ be an eCTRS. Then, $\leftarrow_{R}=\rightarrow_{(R)^{-1}}$.

Proof (Sketch). It suffices to show that $\leftarrow_{(n), R}=\rightarrow_{(n),(R)^{-1}}$ for all $n \geq 0$. This can be proved by induction on $n$.

Regarding the operation $(\cdot)^{-1}$ and the $\mathbb{U}_{\text {opt }}-\mathrm{NE}$ property, the unraveled TRSs are equivalent and we have dual relationships between the $\mathbb{U}_{\text {opt }}-L L$ and $\mathbb{U}_{\text {opt }}-R L$ properties and between the non-LV and non-RV properties.

Theorem 4.7. Let $R$ be an eDCTRS. Then, all of the following hold:

(1) $R$ is $\mathbb{U}_{\mathrm{opt}}-N E$ iff $(R)^{-1}$ is a 3-eDCTRS,

(2) if $R$ is $\mathbb{U}_{\mathrm{opt}}-N E$, then,

a. $\mathbb{U}_{\mathrm{opt}}\left((R)^{-1}\right)=\left(\mathbb{U}_{\mathrm{opt}}(R)\right)^{-1}$ up to the renaming of $U$ symbols (i.e., $U_{i}^{\rho}=U_{k-i+1}^{(\rho)^{-1}}$ for all $1 \leq i \leq k)$,

b. $R$ is $\mathbb{U}_{\mathrm{opt}}-L L$ iff $(R)^{-1}$ is $\mathbb{U}_{\mathrm{opt}}-R L$, and

c. $R$ is $\mathbb{U}_{\mathrm{opt}}-R L$ iff $(R)^{-1}$ is $\mathbb{U}_{\mathrm{opt}}-L L$,

(3) $R$ is non- $L V$ iff $(R)^{-1}$ is non- $R V$, and

(4) $R$ is non- $R V$ iff $(R)^{-1}$ is non- $L V$.

Proof. The proof can be seen in Appendix A.4.

Note that the claim (2)-a in Theorem 4.7 does not hold for $\mathbb{U}$ in general.

Example 4.8. Consider the following $\mathbb{U}_{\mathrm{opt}}-\mathrm{NE} 3$-DCTRS $R_{8}$ and its unraveled TRSs:

$$
\begin{gathered}
R_{8}=\{\quad \mathrm{f}(x) \rightarrow \mathrm{c}(x, y) \Leftarrow \mathrm{g}(x) \rightarrow y ; y \rightarrow \mathrm{h}(x) \quad \mathrm{a} \rightarrow \mathrm{b} \quad \mathrm{g}(\mathrm{a}) \rightarrow \mathrm{h}(\mathrm{b}) \quad\} \\
\mathrm{f}(x) \rightarrow \mathrm{U}_{17}(\mathrm{~g}(x), x) \\
\mathbb{U}_{\text {opt }}\left(R_{8}\right)=\mathbb{U}\left(R_{8}\right)=\left\{\begin{array}{c}
\mathrm{U}_{17}(y, x) \rightarrow \mathrm{U}_{18}(y, x, y) \\
\mathrm{U}_{18}(\mathrm{~h}(x), x, y) \rightarrow \mathrm{c}(x, y) \\
\vdots
\end{array}\right\}
\end{gathered}
$$

The following TRS is obtained from $R_{8}$ by applying $(\cdot)^{-1}$ :

$$
\left(R_{8}\right)^{-1}=\{\quad \mathrm{c}(x, y) \rightarrow \mathrm{f}(x) \Leftarrow \mathrm{h}(x) \rightarrow y ; y \rightarrow \mathrm{g}(x) \quad \mathrm{b} \rightarrow \mathrm{a} \quad \mathrm{h}(\mathrm{b}) \rightarrow \mathrm{g}(\mathrm{a}) \quad\}
$$

The DCTRS $\left(R_{8}\right)^{-1}$ is unraveled by $\mathbb{U}_{\text {opt }}$ and $\mathbb{U}$ as follows:

$$
\begin{array}{r}
\mathbb{U}_{\text {opt }}\left(\left(R_{8}\right)^{-1}\right)=\left\{\begin{aligned}
& \mathrm{c}(x, y) \rightarrow \mathrm{U}_{18}(\mathrm{~h}(x), x, y) \\
& \mathrm{U}_{18}(y, x, y) \rightarrow \mathrm{U}_{17}(y, x) \\
& \mathrm{U}_{17}(\mathrm{~g}(x), x) \rightarrow \mathrm{f}(x) \\
& \vdots
\end{aligned}\right\} \\
\mathbb{U}\left(\left(R_{8}\right)^{-1}\right)=\left\{\begin{array}{c}
\mathrm{c}(x, y) \rightarrow \mathrm{U}_{18}(\mathrm{~h}(x), x, y) \\
\mathrm{U}_{18}(y, x, y) \rightarrow \mathrm{U}_{17}(y, x, y) \\
\mathrm{U}_{17}(\mathrm{~g}(x), x, y) \rightarrow \mathrm{f}(x) \\
\vdots
\end{array}\right\}
\end{array}
$$

We have that $\mathbb{U}_{\text {opt }}\left(\left(R_{8}\right)^{-1}\right)=\left(\mathbb{U}_{\text {opt }}\left(R_{8}\right)\right)^{-1}$, but $\mathbb{U}\left(\left(R_{8}\right)^{-1}\right) \neq\left(\mathbb{U}\left(R_{8}\right)\right)^{-1}$.

Finally, we show soundness of $\mathbb{U}_{\text {opt }}$ for a $\mathbb{U}_{\text {opt }}$-RLNE eDCTRS $R$ by reducing it to soundness for the $\mathbb{U}_{\mathrm{opt}}-\mathrm{LL}$ eDCTRS $(R)^{-1}$. 
Theorem 4.9. $\mathbb{U}_{\mathrm{opt}}$ is sound for $\mathbb{U}_{\mathrm{opt}}-R L N E$ eDCTRSs that are non-LV or non-RV.

Proof. Let $R$ be a $\mathbb{U}_{\text {opt }}$-RLNE eDCTRS over a signature $\mathcal{F}$. Then, it follows from Theorem 4.7 that $(R)^{-1}$ is a $\mathbb{U}_{\text {opt }}$-LL 3-eDCTRS which is non-RV or non-LV. Thus, it follows from Theorem 4.3 that $\mathbb{U}_{\text {opt }}$ is sound for $(R)^{-1}$, i.e., $\rightarrow_{\mathbb{U}_{\mathrm{opt}}\left((R)^{-1}\right)}^{*} \subseteq \rightarrow_{(R)^{-1}}^{*}$ on terms in $\mathcal{T}(\mathcal{F}, \mathcal{V})$. It follows from Theorem 4.7 that $\mathbb{U}_{\text {opt }}\left((R)^{-1}\right)=\left(\mathbb{U}_{\text {opt }}(R)\right)^{-1}$, and hence $\rightarrow_{\mathbb{U}_{\text {opt }}}^{*}\left((R)^{-1}\right)=$ $\rightarrow_{\left(\mathbb{U}_{\mathrm{opt}}(R)\right)^{-1}}^{*}$. It follows from Proposition 4.6 that $\rightarrow_{\left(\mathbb{U}_{\mathrm{opt}}(R)\right)^{-1}}^{*}=\leftarrow_{\mathbb{U}_{\mathrm{opt}}(R)}^{*}$ and $\rightarrow_{(R)^{-1}}^{*}=$ $\leftarrow_{R}^{*}$. Therefore, we have that $\rightarrow_{\mathbb{U}_{\text {opt }}^{*}(R)}^{*} \subseteq \rightarrow_{R}^{*}$ on terms in $\mathcal{T}(\mathcal{F}, \mathcal{V})$.

Example 4.10. Consider the following TRS defining a function quad that computes the quadruple of input natural numbers:

$$
R_{9}=\left\{\begin{array}{l}
\operatorname{quad}(x) \rightarrow \operatorname{twice}(\operatorname{twice}(x)) \\
\operatorname{twice}(x) \rightarrow \operatorname{add}(x, x)
\end{array}\right\} \cup R_{1}
$$

The inversion method in [18] inverts this TRS to the following DCTRS $R_{10}$ :

$$
R_{10}=\left\{\begin{array}{l}
\operatorname{quad}^{-1}(y) \rightarrow \operatorname{tp}_{1}(x) \Leftarrow \operatorname{twice}^{-1}(y) \rightarrow \operatorname{tp}_{1}(z) ; \operatorname{twice}^{-1}(z) \rightarrow \operatorname{tp}_{1}(x) \\
\operatorname{twice}^{-1}(y) \rightarrow \operatorname{tp}_{1}(x) \Leftarrow \operatorname{add}^{-1}(y) \rightarrow \operatorname{tp}_{2}(x, x)
\end{array}\right\} \cup R_{2}
$$

This DCTRS is $\mathbb{U}_{\text {opt }}-\mathrm{RLNE}$, and thus, we recognize from Theorem 4.9 that $\mathbb{U}_{\text {opt }}$ is sound for $R_{10}$, while soundness of $\mathbb{U}_{\text {opt }}$ for the resulting EV-TRSs $\left(e . g ., R_{10}\right)$ of the inversion method [18] has already been shown (cf., [22, 23]). On the other hand, soundness of $\mathbb{U}_{\text {opt }}$ for DCTRSs obtained by removing the unary tuple symbol $\mathrm{tp}_{1}$ that seems meaningless:

$$
R_{10}^{\prime}=\left\{\begin{array}{l}
\text { quad }^{-1}(y) \rightarrow x \Leftarrow \operatorname{twice}^{-1}(y) \rightarrow z ; \operatorname{twice}^{-1}(z) \rightarrow x \\
\operatorname{twice}^{-1}(y) \rightarrow x \Leftarrow \operatorname{add}^{-1}(y) \rightarrow \operatorname{tp}_{2}(x, x)
\end{array}\right\} \cup R_{2}
$$

The soundness results in $\left[18,22,23\right.$, cannot guarantee that $\mathbb{U}_{\text {opt }}$ is sound for $R_{10}^{\prime}$. However, since this DCTRS $R_{10}^{\prime}$ is also $\mathbb{U}_{\text {opt }}-$ RLNE, we recognize from Theorem 4.9 that $\mathbb{U}_{\text {opt }}$ is sound for $R_{10}^{\prime}$.

The open problem mentioned in [18] that $\mathbb{U}_{\text {opt }}$ is sound for $\mathbb{U}_{\text {opt }}-\mathrm{NE}$ eDCTRSs does not hold in general. This indicates that the ultra-NE property on its own is not a soundness condition for either $\mathbb{U}_{\text {opt }}$ or $\mathbb{U}$.

Example 4.11. Consider the 3 -DCTRS $R_{8}$ and the unraveled TRS $\mathbb{U}_{\text {opt }}\left(R_{8}\right)$ in Example4.8 again. $R_{8}$ is $\mathbb{U}_{\text {opt }}-\mathrm{NE}$ and $\mathbb{U}-\mathrm{NE}$, but not $\mathbb{U}_{\text {opt }}-\mathrm{RL}$ or $\mathbb{U}-\mathrm{RL}$, either. We have the derivation $\mathrm{f}(\mathrm{a}) \rightarrow_{\mathbb{U}_{\text {opt }}\left(R_{8}\right)}^{*} \mathrm{c}(\mathrm{b}, \mathrm{h}(\mathrm{b}))$, but $\mathrm{f}(\mathrm{a})$ cannot be reduced by $R_{8}$ to $\mathrm{c}(\mathrm{b}, \mathrm{h}(\mathrm{b}))$. Therefore, $\mathbb{U}_{\text {opt }}$ is not sound for every $\mathbb{U}_{\text {opt }}-\mathrm{NE}$ DCTRS. By the same token, $\mathbb{U}$ is not sound for every $\mathbb{U}-\mathrm{NE}$ DCTRS since $\mathbb{U}_{\text {opt }}\left(R_{8}\right)=\mathbb{U}\left(R_{8}\right)$.

Moreover, the ultra-RL property on its own is not a soundness condition for $\mathbb{U}_{\text {opt }}$.

Example 4.12. Consider the following DCTRS $R_{10}$ and its unraveled TRSs:

$$
\begin{gathered}
R_{10}=\left\{\begin{array}{c}
\mathrm{f}(x) \rightarrow \mathrm{e} \Leftarrow \mathrm{d} \rightarrow \mathrm{I} \\
\mathrm{h}(x, x) \rightarrow \mathrm{A}
\end{array}\right\} \\
\mathbb{U}_{\text {opt }}\left(R_{10}\right)=\left\{\begin{array}{c}
\mathrm{f}(x) \rightarrow \mathrm{U}_{19}(\mathrm{~d}) \\
\mathrm{U}_{19}(\mathrm{I}) \rightarrow \mathrm{e} \\
\vdots
\end{array}\right\} \quad \mathbb{U}\left(R_{10}\right)=\left\{\begin{array}{c}
\mathrm{f}(x) \rightarrow \mathrm{U}_{19}(\mathrm{~d}, x) \\
\mathrm{U}_{19}(\mathrm{I}, x) \rightarrow \mathrm{e} \\
\vdots
\end{array}\right\}
\end{gathered}
$$


The DCTRS $R_{10}$ is $\mathbb{U}_{\text {opt }}-R L$, but not $\mathbb{U}_{\text {opt }}-N E$. Although we have the derivation $\mathrm{h}(\mathrm{f}(\mathrm{a}), \mathrm{f}(\mathrm{b}))$

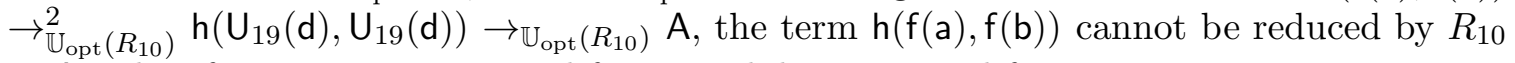
to $\mathrm{A}$. Therefore, $\mathbb{U}_{\text {opt }}$ is not sound for $R_{10}$ while $\mathbb{U}$ is sound for $R_{10}$.

It is possible to prove Theorem 4.9 directly [21], by using the feature that every reduction sequence of RL TRSs can be transformed to a basic reduction sequence [17]. As stated at the end of Subsection 4.2, however, Theorem 4.3 cannot be proved by using Theorem 4.9 since $\mathbb{U}_{\text {opt }}\left((R)^{-1}\right)=\left(\mathbb{U}_{\text {opt }}(R)\right)^{-1}$ does not hold for every $\mathbb{U}_{\text {opt }}$-LL DCTRS $R$ (see $\mathbb{U}_{\text {opt }}\left(R_{10}\right)$ in Example 4.12).

In the proof of Theorem 4.3 (and also the direct proof of Theorem 4.9), linearity plays a very important role and finding other soundness conditions by means of a similar proof scheme is quite difficult.

Finally, we revisit the resulting system of the program inversion mentioned in Section 1 .

Example 4.13. Consider the EV-TRS $\mathbb{U}_{\text {opt }}\left(R_{2}\right)$ in Example 3.2 again. The original TRS $R_{1}$ is left-linear, and thus, $R_{2}$ is $\mathbb{U}_{\text {opt }}-\mathrm{RLNE}\left[18,23\right.$. Theorem 4.9 guarantees that $\mathbb{U}_{\text {opt }}\left(R_{2}\right)$ is an inverse system of $R_{1}$.

4.4. Soundness of Unraveled TRSs with Extra Variables. As we stated in Section 1, the optimized unraveling $\mathbb{U}_{\text {opt }}$ is used in the program inversion method proposed in [18, 22, 23 and DCTRSs obtained by the inversion method are of Type 4 (not of Type 3) in general. For this reason, in this subsection, we extend Theorem 4.3 to 4 -eDCTRSs. More precisely, we show that $\mathbb{U}_{\text {opt }}$ is sound for $\mathbb{U}_{\text {opt }}-\mathrm{LL}$ DCTRSs if reduction sequences of the unraveled TRSs are restricted to $E V$-safe reduction sequences (see Definition 4.14). Roughly speaking, in an EV-safe reduction sequence, any redex introduced via extra variables at the application of rewrite rules is never reduced anywhere. In practical cases (e.g., inverse TRSs [18, 22, 23, 20]), extra variables are instantiated with constructor terms. However, at the application of rewrite rules, extra variables in the unraveled eTRSs may introduce undesired terms, e.g., terms rooted by U symbols that are not reachable from terms over the original signature. As a consequence, $\mathbb{U}_{\text {opt }}$ is not always sound w.r.t. non-EV-safe reduction sequences of the unraveled eTRSs (see Example 4.18).

We first define the notion of $E V$-safe reduction sequences of eTRSs [20, 18, 21]. This notion can be formalized by extending the notion of basic reduction sequences [11, 17].

Definition 4.14 (EV-safe reduction [20]). Let $R$ be an eTRS and $\rho_{i}: l_{i} \rightarrow r_{i} \in R$ for all $i$ $\geq 1$. Let $t_{0} \rightarrow_{p_{1}, \rho_{1}} t_{2} \rightarrow_{p_{2}, \rho_{2}} \cdots$ be a reduction sequence of $R$, and $B_{0} \subseteq \mathcal{P}_{\text {osf }}\left(t_{0}\right)$ such that $B_{0}$ is prefix closed (i.e., if $p<q$ and $q \in B_{0}$, then $p \in B_{0}$ ). We define the sets $B_{1}, B_{2}, \ldots$ of positions from the sequence and $B_{0}$ inductively as

$$
\begin{aligned}
B_{i}= & \left(B_{i-1} \backslash\left\{q \in B_{i-1} \mid q \geq p_{i}\right\}\right) \cup\left\{p_{i} q \mid q \in \mathcal{P}_{o s_{\mathcal{F}}}\left(r_{i}\right)\right\} \\
& \cup\left\{p_{i} p^{\prime} q\left|p_{i} p q \in B_{i-1}, p \in \mathcal{P}_{\text {os }}\left(l_{i}\right), l_{i}\right|_{p}=\left.r_{i}\right|_{p^{\prime}}\right\}
\end{aligned}
$$

for all $i \geq 1$. Note that $B_{1}, B_{2}, \ldots$ are also prefix closed. For all $i \geq 0$, positions in $B_{i}$ are referred as basic positions of $t_{i}$ w.r.t. extra variables. The reduction sequence above is said to be based on $B_{0}$ w.r.t. extra variables if $p_{i} \in B_{i-1}$ for all $i \geq 1$. If the sequence is finite with length $n$, then we denote it by $B_{0}: t_{0} \overrightarrow{\operatorname{evs}}_{R}^{*} B_{n}: t_{n}$ or $B_{0}: t_{0} \overrightarrow{\mathrm{evs}}_{R}^{*} t_{n}$. In particular, the reduction sequence is called safe w.r.t. extra variables (EV-safe) if $B_{0}=\mathcal{P}_{o s}\left(t_{0}\right)$. If the EV-safe sequence is finite with length $n$, then we denote it by $t_{0} \overrightarrow{\mathrm{evs}}_{R}^{*} t_{n}$. 
Note that EV-safeness is different from basicness [11, 17] in the sense that all the basic positions are propagated at the application of rewrite rules, but none of the positions for extra variables are added to basic positions. A typical instance of EV-safe reduction sequences is a reduction sequence obtained by substituting a normal form for each extra variable when applying rewrite rules.

To specify a set of terms that extra variables are possibly instantiated at the rule application, we introduce the notion of $E V$-instantiation on sets of terms. Let $R$ be an eTRS and $T$ be a set of terms. A derivation $t_{0} \rightarrow_{p_{1}, \rho_{1}} t_{1} \rightarrow_{p_{2}, \rho_{2}} \cdots$ of $R$ is called $E V$ instantiated on $T$ if any extra variable of $\rho_{i}: l_{i} \rightarrow r_{i}$ is instantiated by a term in $T$, i.e., $\left.t_{i}\right|_{p_{i} q} \in T$ for any $q \in \mathcal{P} \operatorname{Po} \mathcal{V}\left(r_{i}\right)$ such that $\left.r_{i}\right|_{q} \in \mathcal{E} \mathcal{V} \operatorname{ar}\left(\rho_{i}\right)$. By the same token, the notion of the EV-instantiation property is defined for the parallel reduction of eTRSs. For any of the unraveled eTRSs, their EV-safe reduction sequences have the following property related to EV-instantiation on the set of terms over the original signature.

Lemma 4.15. Let $R$ be a $\mathbb{U}_{\mathrm{opt}}-L L$ eDCTRS over a signature $\mathcal{F}$, and $s, t$ be terms in

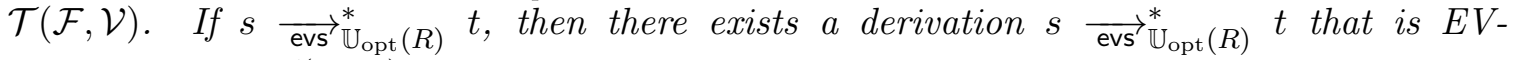
instantiated on $\mathcal{T}(\mathcal{F}, \mathcal{V})$.

Proof. The proof can be seen in Appendix A.5.

Lemma 4.2, the key lemma for the case of $\mathbb{U}_{\text {opt }}$-LL 3-DCTRSs, is adapted to 4-eDCTRSs as follows.

Lemma 4.16. Let $R$ be a $\mathbb{U}_{\mathrm{opt}}-L L$ eDCTRS over a signature $\mathcal{F}$, $s$ be a term in $\mathcal{T}(\mathcal{F}, \mathcal{V})$, $t$ be a linear term in $\mathcal{T}\left(\mathcal{F}_{\mathbb{U}_{\text {opt }}(R)}, \mathcal{V}\right)$, and $\sigma$ be a substitution in $\mathcal{S} u b\left(\mathcal{F}_{\mathbb{U}_{\text {opt }}(R)}, \mathcal{V}\right)$. Suppose

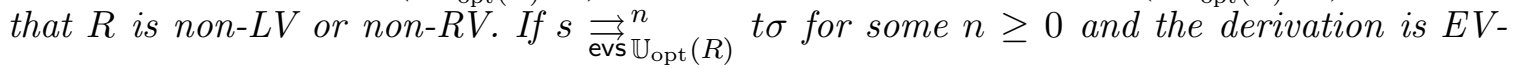
instantiated on $\mathcal{T}(\mathcal{F}, \mathcal{V})$, then there exists a substitution $\theta$ in $\mathcal{S} u b(\mathcal{F}, \mathcal{V})$ such that

- $s \rightarrow_{R}^{*} t \theta \underset{\mathrm{evs}}{\rightrightarrows} n_{\mathcal{P}^{\prime}}^{n_{\mathcal{V}}(t), \mathbb{U}_{\mathrm{opt}}(R)}$ to for some $n^{\prime} \leq n$,

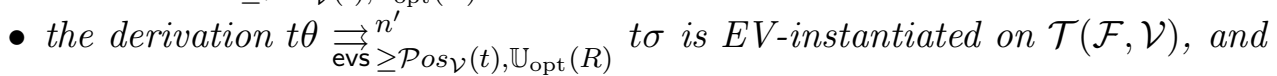

- if $t \sigma \in \mathcal{T}(\mathcal{F}, \mathcal{V})$, then $t \theta=t \sigma$.

Proof. This lemma can be proved similarly to Lemma 4.2.

As a consequence of Lemma 4.16, we extend Theorem 4.3 to 4-eDCTRSs.

Theorem 4.17. $\mathbb{U}_{\mathrm{opt}}$ is sound for a $\mathbb{U}_{\mathrm{opt}}-L L$ eDCTRSs $R$ over a signature $\mathcal{F}$ w.r.t. $\overrightarrow{\mathrm{evs}} \mathbb{U}_{\mathrm{opt}}(R)$ if $R$ is non- $L V$ or non- $R V$.

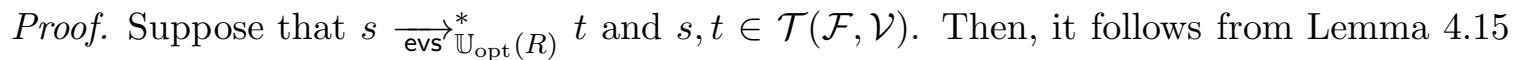
that there is a derivation $s \underset{\text { evs }}{\mathbb{U}_{\text {opt }}(R)}$ that is EV-instantiated on $\mathcal{T}(\mathcal{F}, \mathcal{V})$. Since a single step of $\overrightarrow{\text { evs }}_{\mathbb{U}_{\text {opt }}(R)}$ can be considered a single step of the parallel reduction, we have the derivation $s \underset{\text { evs }}{\stackrel{\text { opt }}{\longrightarrow}(R)}$ t that is EV-instantiated on $\mathcal{T}(\mathcal{F}, \mathcal{V})$. Let $x$ be a variable and $\sigma$ be a substitution such that $x \sigma=t$. Then, it follows from Lemma 4.16 that $s \rightarrow_{R}^{*} x \sigma=t$.

Note that Lemma 4.16 and Theorem 4.17 are strict extensions of Lemma 4.2 and Theorem 4.3 , respectively.

Finally, we show a counterexample against Theorem 4.3 without the EV-safe property. 
Example 4.18. Consider the DCTRS $R_{10}$ and its unraveled TRSs in Example 4.12 again. Their inverted systems are as follows:

$$
\begin{gathered}
\left(R_{10}\right)^{-1}=\left\{\begin{array}{c}
\mathrm{e} \rightarrow \mathrm{f}(x) \Leftarrow \mathrm{I} \rightarrow \mathrm{d} \\
\mathrm{A} \rightarrow \mathrm{h}(x, x)
\end{array}\right\} \\
\mathbb{U}_{\text {opt }}\left(\left(R_{10}\right)^{-1}\right)=\mathbb{U}\left(\left(R_{10}\right)^{-1}\right)=\left(\mathbb{U}_{\text {opt }}\left(R_{10}\right)\right)^{-1}=\left\{\begin{array}{c}
\mathrm{e} \rightarrow \mathrm{U}_{19}(\mathrm{I}) \\
\mathrm{U}_{19}(\mathrm{~d}) \rightarrow \mathrm{f}(x) \\
\vdots
\end{array}\right\}
\end{gathered}
$$

We have the derivation $\mathrm{A} \rightarrow \mathbb{U}_{\mathrm{opt}}\left(\left(R_{10}\right)^{-1}\right) \mathrm{h}\left(\mathrm{U}_{19}(\mathrm{~d}), \mathrm{U}_{19}(\mathrm{~d})\right) \rightarrow_{\mathbb{U}_{\text {opt }}\left(\left(R_{10}\right)^{-1}\right)}^{*} \mathrm{~h}(\mathrm{f}(\mathrm{a}), \mathrm{f}(\mathrm{b}))$ that is not EV-safe: the term $\mathrm{U}_{19}(\mathrm{~d})$ introduced by instantiating the extra variable $x$ in the applied rule $\mathrm{A} \rightarrow \mathrm{h}(x, x)$ is reduced. However, A cannot be reduced by $\left(R_{10}\right)^{-1}$ to $\mathrm{h}(\mathrm{f}(\mathrm{a}), \mathrm{f}(\mathrm{b}))$. Therefore, $\mathbb{U}_{\text {opt }}$ is not sound for $\left(R_{10}\right)^{-1}$. Note that $\mathbb{U}$ is not sound for $\left(R_{10}\right)^{-1}$, either.

Note that if an unraveling $U$ is sound for an eCTRS $R$, then $U$ is sound for $R$ w.r.t. $\overrightarrow{\mathrm{evs}} U(R)$. For this reason, we need not discuss soundness of $U$ for $R$ w.r.t. $\overrightarrow{\operatorname{evs}} U(R)$ when soundness of $U$ for $R$ w.r.t. $\rightarrow_{U(R)}$ has already been shown.

\section{Soundness of Other UnRAVElings}

In this section, we show that soundness of $\mathbb{U}_{\text {opt }}$ implies that of $\mathbb{U}$, and then we revisit soundness of the unravelings for join and normal CTRSs.

We first recall the notion of tree homomorphisms. Let $\mathcal{F}$ and $\mathcal{G}$ be signatures and $\phi_{\mathcal{F}}$ be a mapping which, for an $n$-ary function symbol $f \in \mathcal{F}$, associates a term in $\mathcal{T}\left(\mathcal{G},\left\{x_{1}, \ldots, x_{n}\right\}\right)$ where $x_{1}, \ldots, x_{n} \in \mathcal{V}$. The tree homomorphism $\phi: \mathcal{T}(\mathcal{F}, \mathcal{V}) \rightarrow \mathcal{T}(\mathcal{G}, \mathcal{V})$ determined by $\phi_{\mathcal{F}}$ is defined as follows [33, 5]:

- $\phi(x)=x$ for $x \in \mathcal{V}$, and

- $\phi\left(f\left(t_{1}, \ldots, t_{n}\right)\right)=\phi_{\mathcal{F}}(f)\left\{x_{i} \mapsto \phi\left(t_{i}\right) \mid 1 \leq i \leq n\right\}$ for an $n$-ary function symbol $f \in \mathcal{F}$.

When $\phi_{\mathcal{F}}(f)$ is not specified explicitly for an $n$-ary function symbol $f$, we let $\phi_{\mathcal{F}}(f)=$ $f\left(x_{1}, \ldots, x_{n}\right)$ with assuming that $f \in \mathcal{G}$. To declare $\phi_{\mathcal{F}}$ intelligibly, we may use the notation " $\phi_{\mathcal{F}}\left(f\left(x_{1}, \ldots, x_{n}\right)\right)=t$ " instead of " $\phi_{\mathcal{F}}(f)=t$ ", e.g., $\phi_{\mathcal{F}}(\mathrm{f}(x, y, z))=\mathrm{g}(y, \mathrm{~g}(x, z))$. The tree homomorphism $\phi$ is called linear if $\phi_{\mathcal{F}}(f)$ is linear for any function symbol $f \in \mathcal{F}$, and called non-erasing if $\mathcal{V} a r(\phi(f))=\left\{x_{1}, \ldots, x_{n}\right\}$ for any $n$-ary function symbol $f \in \mathcal{F}$. The tree homomorphism $\phi$ is extended to eCTRSs as follows: $\phi(R)=\left\{\phi(l) \rightarrow \phi(r) \Leftarrow \phi\left(s_{1}\right)=\right.$ $\left.\phi\left(t_{1}\right) ; \ldots ; \phi\left(s_{k}\right)=\phi\left(t_{k}\right) \mid l \rightarrow r \Leftarrow s_{1} \approx t_{1} ; \ldots ; s_{k} \approx t_{k} \in R\right\}$. We extend it to a set of term pairs $T$ (e.g., a binary relation) as follows: $\phi(T)=\{(\phi(s), \phi(t)) \mid(s, t) \in T\}$. For a substitution $\sigma \in \mathcal{S} u b(\mathcal{F}, \mathcal{V}), \sigma_{\phi}$ denotes the substitution $\{x \mapsto \phi(x \sigma) \mid x \in \operatorname{Dom}(\sigma)\}$. Tree homomorphisms have the following properties.

Lemma 5.1. Let $\phi$ be a tree homomorphism.

(1) Let $t$ be a term and $\sigma$ be a substitution in $\mathcal{S} u b(\mathcal{F}, \mathcal{V})$. Then, $\phi(t \sigma)=(\phi(t)) \sigma_{\phi}$.

(2) Let $t$ be a term and $C[]$ be a one-hole context. Then, all of the following hold:

- $\phi(C[t])=\phi(C)[\overbrace{\phi(t), \ldots, \phi(t)}^{n}]$

- if $\phi$ is non-erasing, then $\phi(C)$ has at least one hole,

\footnotetext{
${ }^{4} \phi(C)$ has no hole (i.e., $\left.n=0\right)$ if $\phi$ removes the hole from $C[]$.
} 
- if $\phi$ is linear, then $\phi(C)$ has at most one hole. That is, if $\phi$ is linear, then, for any term $t$ and any one-hole context $C[]$, either $\phi(C[t])=\phi(C)$ or $\phi(C[t])=\phi(C)[\phi(t)]$.

(3) Let $R$ be an eCTRS and $s, t$ be terms in $\mathcal{T}(\mathcal{F}, \mathcal{V})$. If $s \rightarrow_{R}^{*} t$, then $\phi(s) \rightarrow_{\phi(R)}^{*} \phi(t)$. That is, $\phi\left(\rightarrow_{R}^{*}\right) \subseteq \rightarrow_{\phi(R)}^{*}$.

The proof of Lemma 5.1 is omitted since it can be easily proved by induction.

5.1. Abstract Comparison Method for Soundness of Unravelings. Before we discuss the relationship between soundness of two or more unravelings, we present a sufficient condition of two unravelings under which soundness of the first implies soundness of the other.

To show soundness of an unraveling $U_{2}$ by means of a sound unraveling $U_{1}$, it suffices to show that all the derivations of $U_{2}$ on terms over the original signature are included in the derivations of $U_{1}$, i.e., $\rightarrow_{U_{2}(R)}^{*} \subseteq \rightarrow_{U_{1}(R)}^{*}$ on terms in $\mathcal{T}(\mathcal{F}, \mathcal{V})$. Suppose that $U_{1}$ is sound for a CTRS R, i.e., $\rightarrow_{U_{1}(R)}^{*} \subseteq \rightarrow_{R}^{*}$ on terms in $\mathcal{T}(\mathcal{F}, \mathcal{V})$. Then, it follows from $\rightarrow_{U_{2}(R)}^{*} \subseteq$ $\rightarrow_{U_{1}(R)}^{*}$ that $\rightarrow_{U_{2}(R)}^{*} \subseteq \rightarrow_{R}^{*}$ on terms in $\mathcal{T}(\mathcal{F}, \mathcal{V})$. Therefore, $U_{2}$ is sound for $R$.

To show that $\rightarrow_{U_{2}(R)}^{*} \subseteq \rightarrow_{U_{1}(R)}^{*}$ on terms in $\mathcal{T}(\mathcal{F}, \mathcal{V})$, it suffices to show the existence of a tree homomorphism $\phi$ for an extended signature of $\mathcal{F}$ such that

- $U_{1}(R)=\phi\left(U_{2}(R)\right)$, and

- $\phi(t)=t$ for any term $t \in \mathcal{T}(\mathcal{F}, \mathcal{V})$.

Moreover, since we consider soundness w.r.t. $\overrightarrow{\text { evs }}$, we are interested in a sufficient condition under which $\overrightarrow{\mathrm{evs}}_{U_{2}(R)}^{*} \subseteq \overrightarrow{\mathrm{evs}}_{U_{1}(R)}^{*}$. To show that $\overrightarrow{\mathrm{evs}}_{U_{2}(R)}^{*} \subseteq \overrightarrow{\mathrm{evs}}_{U_{1}(R)}^{*}$, it suffices to additionally show that $\mathcal{E} \mathcal{V}$ ar $(\phi(l) \rightarrow \phi(r)) \subseteq \mathcal{E} \mathcal{V}$ ar $(l \rightarrow r)$ for any rule $l \rightarrow r \in U_{2}(R)$.

For a set $\mathcal{G} \subseteq \mathcal{F}$ of function symbols, a tree homomorphism $\phi$ determined by a mapping $\phi_{\mathcal{F}}$ is called $\mathcal{G}$-identical if $\phi_{\mathcal{F}}(f)=f\left(x_{1}, \ldots, x_{n}\right)$ for any $n$-ary function symbol $f \in \mathcal{G}$. Moreover, $\phi$ is called $E V$-preserving for an eTRS $R$ if $\mathcal{E} \mathcal{V}$ ar $(\phi(l) \rightarrow \phi(r))=\mathcal{E} \mathcal{V}$ ar $(l \rightarrow r)$ for any rule $l \rightarrow r \in R$.

Lemma 5.2. Let $R$ be an eTRS, $s, t$ be terms, and $\phi$ be a tree homomorphism that is $E V$-preserving for $R$. If $s \overrightarrow{\mathrm{evs}}_{R}^{*} t$, then $\phi(s) \overrightarrow{\mathrm{evs}}_{\phi(R)}^{*} \phi(t)$.

Proof. We first define the mapping $\phi_{u}$ from a position of a term $u$ to a set of positions of $\phi(u)$, and extend it to sets of positions of $u$ :

- $\phi_{x}(\varepsilon)=\{\varepsilon\}$ for $x \in \mathcal{V}$,

- $\phi_{f\left(u_{1}, \ldots, u_{n}\right)}(\varepsilon)=\{\varepsilon\}$ for an $n$-ary function symbol $f$,

- $\phi_{f\left(u_{1}, \ldots, u_{n}\right)}(i p)=\left\{q p^{\prime}\left|q \in \mathcal{P}_{o s} \mathcal{V}\left(\phi_{\mathcal{F}}(f)\right), \phi_{\mathcal{F}}(f)\right|_{q}=x_{i}, p^{\prime} \in \phi_{u_{i}}(p)\right\}$ for an $n$-ary function symbol $f$, where $1 \leq i \leq n$ and $p \in \mathcal{P}$ os $\left(u_{i}\right)$, and

- $\phi_{u}(P)=\bigcup_{p \in P} \phi_{u}(p)$.

For a position $p$ of $u$, we mean by $p^{\prime} \in \phi_{u}(p)$ that the application of $\phi$ to $u$ maps $\left.u\right|_{p}$ to $\left.\phi(u)\right|_{p^{\prime}}$. Note that positions in $\phi_{u}(p)$ are parallel since variable positions of $\phi_{\mathcal{F}}(f)$ are parallel. We prove that if $B: s \overrightarrow{\mathrm{evs}}_{R}^{*} B^{\prime}: t$ and $\phi_{s}(B) \subseteq B_{1} \subseteq \mathcal{P}$ os $(\phi(s))$, then $B_{1}: \phi(s)$ $\overrightarrow{\text { evs }}_{\phi(R)}^{*} B_{1}^{\prime}: \phi(t)$ and $\phi_{t}\left(B^{\prime}\right) \subseteq B_{1}^{\prime}$. To prove this claim by induction on the length $n$ of the derivation $B: s \overrightarrow{\mathrm{evs}}_{R}^{*} B^{\prime}: t$, it suffices to show that if $B: s \overrightarrow{\operatorname{evs}}_{p, R} B^{\prime}: t$, then $\phi_{s}(p)$ is defined and $\phi_{s}(B): \phi(s) \overrightarrow{\mathrm{evs}}_{q_{1}, \phi(R)} \cdots \overrightarrow{\mathrm{evs}}_{q_{m}, \phi(R)} \phi_{t}\left(B^{\prime}\right): \phi(t)$ where $\phi_{s}(p)=\left\{q_{1}, \ldots, q_{m}\right\}$. This follows from the assumption and the definitions of $\underset{\mathrm{evs}}{\longrightarrow}$ and $\phi_{t}$. 
Accordingly, to show soundness of $U_{2}$ by means of soundness of $U_{1}$, we obtain the following useful lemma.

Lemma 5.3. Let $U_{1}$ and $U_{2}$ be unravelings, $R$ be an eCTRS over a signature $\mathcal{F}$, and $\mathcal{G}$ be an extended signature of $\mathcal{F}$ such that $U_{2}(R)$ is defined over $\mathcal{G}$. Let $\phi$ be an $\mathcal{F}$-identical tree homomorphism determined by $\phi_{\mathcal{G}}$ such that $U_{1}(R)=\phi\left(U_{2}(R)\right)$. Then, all of the following hold:

$(1) \rightarrow_{U_{2}(R)}^{*} \subseteq \rightarrow_{U_{1}(R)}^{*}$ on terms in $\mathcal{T}(\mathcal{F}, \mathcal{V})$,

(2) if $\phi$ is non-erasing, then $\phi$ is $E V$-preserving for any eTRS, and

(3) if $\phi$ is EV-preserving for $U_{2}(R)$, then $\overrightarrow{\mathrm{evs}}_{U_{2}(R)}^{*} \subseteq \underset{\mathrm{evs}}{U_{1}(R)}$ on terms in $\mathcal{T}(\mathcal{F}, \mathcal{V})$.

That is, all of the following holds:

(4) if $U_{1}$ is sound for $R$, then so is $U_{2}$,

(5) if $\phi$ is non-erasing and $U_{1}$ is sound for $R$ w.r.t. $\overrightarrow{\mathrm{evs}}_{U_{1}(R)}$, then $U_{2}$ is sound for $R$ w.r.t. $\overrightarrow{\mathrm{evs}} U_{2}(R)$, and

(6) if $\phi$ is $E V$-preserving for $U_{2}(R)$ and $U_{1}$ is sound for $R$ w.r.t. $\overrightarrow{\mathrm{evs}} U_{1}(R)$, then $U_{2}$ is sound for $R$ w.r.t. $\overrightarrow{\mathrm{evs}} U_{2}(R)$.

Proof. We first prove the first claim $\rightarrow_{U_{2}(R)}^{*} \subseteq \rightarrow_{U_{1}(R)}^{*}$ on terms in $\mathcal{T}(\mathcal{F}, \mathcal{V})$. It follows from Lemma 5.1 and the assumption $U_{1}(R)=\phi\left(U_{2}(R)\right)$ that $\phi\left(\rightarrow_{U_{2}(R)}^{*}\right) \subseteq \rightarrow_{U_{1}(R)}^{*}$. Since $\phi$ is $\mathcal{F}$-identical, we have that $\rightarrow_{U_{2}(R)}^{*} \subseteq \rightarrow_{U_{1}(R)}^{*}$ on terms in $\mathcal{T}(\mathcal{F}, \mathcal{V})$.

Let $\phi$ be determined by a mapping $\phi_{\mathcal{G}}$. To prove the second claim, it suffices to show that $\operatorname{Var}(t)=\mathcal{V} \operatorname{ar}(\phi(t))$ for any term $t$. We prove this claim by induction on the structure of $t$. Since the case that $t$ is a variable is trivial, we only consider the remaining case that $t$ is of the form $f\left(t_{1}, \ldots, t_{n}\right)$. By the induction hypothesis, $\operatorname{Var}\left(t_{i}\right)=\operatorname{Var}\left(\phi\left(t_{i}\right)\right)$ for all $1 \leq i \leq n$. It follows from the non-erasingness of $\phi$ that $\operatorname{Var}\left(\phi\left(f\left(t_{1}, \ldots, t_{n}\right)\right)\right)=$ $\mathcal{V} a r\left(\phi_{\mathcal{G}}(f)\left\{x_{i} \mapsto \phi\left(t_{i}\right) \mid 1 \leq i \leq n\right\}\right)=\bigcup_{i=1}^{n} \operatorname{Var}\left(\phi\left(t_{i}\right)\right)$, and hence $\operatorname{Var}(\phi(t))=\mathcal{V} a r(t)$.

The third claim follows from the first claim and Lemma 5.2. The remaining claims follow from the first, second, and third claims, and soundness of $U_{1}$.

Due to Lemma 5.3, to show soundness of $U_{2}$ by soundness of $U_{1}$, it suffices to show the existence of an $\mathcal{F}$-identical tree homomorphism $\phi$ satisfying that $U_{1}(R)=\phi\left(U_{2}(R)\right)$. Moreover, for the case of soundness w.r.t. $\underset{\text { evs }}{\longrightarrow}$, it suffices to additionally show that the tree homomorphism $\phi$ is non-erasing or EV-preserving for $U_{2}(R)$.

5.2. On Ohlebusch's Unraveling for DCTRSs. As stated in Section 3, the optimized unraveling $\mathbb{U}_{\text {opt }}$ is a variant of the unraveling $\mathbb{U}$, in the sense that variables carried by $U$ symbols are optimized. For this reason, for a DCTRS $R$, it is easy to find a tree homomorphism $\phi$ such that $\mathbb{U}_{\text {opt }}(R)=\phi(\mathbb{U}(R))$. In the following, we assume that for every rule $\rho \in$ $R$, the same $U$ symbols $U_{1}^{\rho}, \ldots, U_{k}^{\rho}$ are introduced for $\mathbb{U}_{\text {opt }}(\rho)$ and $\mathbb{U}(\rho)$.

Lemma 5.4. Let $R$ be an eDCTRS over a signature $\mathcal{F}$. There exists an $\mathcal{F}$-identical tree homomorphism $\phi$ such that $\phi(\mathbb{U}(R))=\mathbb{U}_{\text {opt }}(R)$ and $\phi$ is EV-preserving for $\mathbb{U}(R)$.

Proof. Let $\phi$ be a tree homomorphism determined by $\phi_{\mathcal{F}_{\mathbb{U}(R)}}$ such that

$$
\phi_{\mathcal{F}_{\mathbb{U}(R)}}\left(U_{i}^{\rho}\left(x_{i}, \vec{X}_{i}\right)\right)=U_{i}^{\rho}\left(x_{i}, \vec{Z}_{i}\right)
$$

where $x_{i}$ is a fresh variable such that $x_{i} \notin X_{i}$. Then, it is clear that $\phi(\mathbb{U}(\rho))=\mathbb{U}_{\text {opt }}(\rho)$, and hence $\phi(\mathbb{U}(R))=\mathbb{U}_{\text {opt }}(R)$. 
Next, we show that $\phi_{\mathcal{F}_{\mathbb{U}(R)}}$ is EV-preserving for $\mathbb{U}(R)$. For unconditional rules $l_{0} \rightarrow r_{0}$ in $R$, it is clear that $\mathcal{E} \mathcal{V} a r\left(l_{0} \rightarrow r_{0}\right)=\mathcal{E} \mathcal{V} a r\left(\phi\left(l_{0}\right) \rightarrow \phi\left(r_{0}\right)\right)$, since $l_{0}, r_{0} \in \mathcal{T}(\mathcal{F}, \mathcal{V})$ and $\phi(t)=t$ for all $t \in \mathcal{T}(\mathcal{F}, \mathcal{V})$. Thus, we only consider the case of conditional rules $\rho$ : $l \rightarrow r \Leftarrow s_{1} \rightarrow t_{1} ; \ldots ; s_{k} \rightarrow t_{k} \in R$. Rules in $\mathbb{U}_{\text {opt }}(\rho)$ and $\mathbb{U}(\rho)$ that may contain extra variables are rules of $U_{k}^{\rho}$, that is, $\rho_{k}: U_{k}^{\rho}\left(t_{k}, \overrightarrow{Z_{k}}\right) \rightarrow r \in \mathbb{U}_{\text {opt }}(R), \rho_{k}^{\prime}: U_{k}^{\rho}\left(t_{k}, \overrightarrow{X_{k}}\right) \rightarrow r$ $\in \mathbb{U}(R)$. It follows from $X_{k} \cap Y_{k} \subseteq X_{k}$ and $\mathcal{V} a r(r) \cap Z_{k}=\operatorname{Var}(r) \cap X_{k}$ that $\mathcal{E} \operatorname{Var}\left(\rho_{k}\right)=$ $\mathcal{V} a r(r) \backslash\left(\mathcal{V} a r\left(t_{k}\right) \cup\left(\mathcal{V} a r\left(l, t_{1}, \ldots, t_{k-1}\right) \cap \mathcal{V} a r\left(r, t_{k}\right)\right)\right)=\mathcal{V} a r(r) \backslash \mathcal{V} a r\left(l, t_{1}, \ldots, t_{k}\right)=\mathcal{E} \mathcal{V} a r\left(\rho_{k}^{\prime}\right)$. Therefore, $\phi_{\mathcal{F}_{\mathbb{U}(R)}}$ is EV-preserving for $\mathbb{U}(R)$.

As a consequence, we conclude that soundness of $\mathbb{U}_{\text {opt }}$ implies soundness of $\mathbb{U}$.

Corollary 5.5. Let $R$ be an eDCTRS over a signature $\mathcal{F} . \mathbb{U}$ is sound for $R$ (w.r.t. $\overrightarrow{\mathrm{evs}}_{\mathbb{U}(R)}$ ) if $\mathbb{U}_{\mathrm{opt}}$ is sound for $R$ (w.r.t. $\overrightarrow{\mathrm{evs}}_{\mathbb{U}_{\mathrm{opt}}(R)}$ ).

Example 5.6. Consider the DCTRS $R_{10}^{\prime}$ in Example 4.10 again. As stated in Example 5.6, $\mathbb{U}_{\text {opt }}$ is sound for $R_{10}^{\prime}$, and thus, we recognize from Corollary 5.5 that $\mathbb{U}$ is also sound for $R_{10}^{\prime}$.

The converse of Corollary 5.5 does not hold in general since, for a DCTRS $R$ over a signature $\mathcal{F}, \rightarrow_{\mathbb{U}(R)}^{*} \neq \rightarrow \rightarrow_{\mathbb{U}_{\text {opt }}(R)}^{*}$ on terms in $\mathcal{T}(\mathcal{F}, \mathcal{V})$ in general (see Example4.12). The reason why the converse of Corollary 5.5 does not hold must be that the U symbols introduced via the application of $\mathbb{U}$ have more variables (i.e., information) than the corresponding $U$ symbols introduced by $\mathbb{U}_{\text {opt }}$. Thus, $\mathbb{U}$ is sufficient to produce TRSs that can be used instead of the original DCTRSs. Nonetheless, $\mathbb{U}_{\text {opt }}$ will be useful in investigating soundness of $\mathbb{U}$ since the unraveled TRSs obtained by $\mathbb{U}_{\text {opt }}$ are simpler than those obtained by $\mathbb{U}$.

5.3. On Unravelings for Join and Normal CTRSs. Join CTRSs can be converted into equivalent normal CTRSs that are special cases of DCTRSs, and normal CTRSs are join CTRSs since the conditions $s_{i} \rightarrow n_{i}$ and $s_{i} \downarrow n_{i}$ are identical:

$$
\text { join } \mathrm{CTRS}=\text { normal CTRS } \subset \text { DCTRS }
$$

In this subsection, we show that the unraveling $\mathbb{U}_{\mathrm{J}}$ for join CTRSs [15] is sound for join CTRSs if the unraveling $\mathbb{U}_{\mathrm{N}}$ for normal CTRSs [26, 9] is sound for the corresponding normal CTRSs. Then, by using this result and the existing soundness condition of $\mathbb{U}_{N}[9]$, we show that $\mathbb{U}_{\mathrm{J}}$ is sound for LL join CTRSs. We also show that $\mathbb{U}_{\mathrm{J}}$ is sound for join CTRSs that can be considered normal CTRSs. Moreover, we show that $\mathbb{U}_{\mathrm{N}}$ is sound for a normal CTRS if $\mathbb{U}_{\mathrm{J}}$ is sound for the normal CTRS that is considered as a join CTRS. Finally, we show that soundness of $\mathbb{U}_{N}$ implies soundness of $\mathbb{U}$. As far as we know, soundness of $\mathbb{U}_{\mathrm{J}}$ has never been discussed, whereas soundness of $\mathbb{U}_{\mathrm{N}}$ has been investigated in some papers [15, 9]. For this reason, we show the soundness condition for $\mathbb{U}_{\mathrm{J}}$ and compare soundness of $\mathbb{U}_{\mathrm{J}}$ with $\mathbb{U}_{\mathrm{N}}$.

A CTRS $R$ is called join if the symbol $\approx$ in the conditions of rewrite rules is interpreted as joinability: the reduction relation of $R$ is defined as $\rightarrow_{R}=\bigcup_{n \geq 0} \rightarrow_{(n), R}$ where

- $\rightarrow(0), R=\emptyset$, and

- $\rightarrow_{(i+1), R}=\rightarrow_{(i), R} \cup\left\{\left(C[l \sigma]_{p}, C[l \sigma]_{p}\right) \mid \rho: l \rightarrow r \Leftarrow s_{1} \approx t_{1} ; \ldots ; s_{k} \approx t_{k} \in R, s_{1} \sigma \downarrow_{(i), R}\right.$ $\left.t_{1} \sigma, \ldots, s_{k} \sigma \downarrow_{(i), R} t_{k} \sigma\right\}$ for $i \geq 0$.

From now on, rewrite rules $l \rightarrow r \Leftarrow s_{1} \approx t_{1} ; \ldots ; s_{k} \approx t_{k}$ of join CTRSs are written as $l \rightarrow r \Leftarrow s_{1} \downarrow t_{1} ; \ldots ; s_{k} \downarrow t_{k}$. 
We first recall the definition of the unravelings $\mathbb{U}_{\mathrm{J}}$ and $\mathbb{U}_{\mathrm{N}}$ for join and normal CTRSs, that are variants of unravelings proposed by Marchiori [15].

Definition $5.7\left(\mathbb{U}_{\mathrm{J}}, \mathbb{U}_{\mathrm{N}}[26,9]\right)$. Let $R$ be a join or normal eCTRS over a signature $\mathcal{F}$. Introducing a $\mathrm{U}$ symbol $U^{\rho}$, we transform $\rho: l \rightarrow r \Leftarrow s_{1} \approx t_{1} ; \ldots ; s_{k} \approx t_{k}$ into sets $\mathbb{U}_{\mathrm{J}}(\rho)$ and $\mathbb{U}_{\mathrm{N}}(\rho)$ of two unconditional rules as follows:

- $\mathbb{U}_{\mathrm{J}}(\rho)=\left\{l \rightarrow U^{\rho}\left(s_{1}, t_{1}, \ldots, s_{k}, t_{k}, \overrightarrow{\mathcal{V} a r(l)}\right), U^{\rho}\left(x_{1}, x_{1}, \ldots, x_{k}, x_{k}, \overrightarrow{\mathcal{V} a r(l)}\right) \rightarrow r\right\}$ if $R$ is join, and

- $\mathbb{U}_{\mathrm{N}}(\rho)=\left\{l \rightarrow U^{\rho}\left(s_{1}, \ldots, s_{k}, \overrightarrow{\mathcal{V} a r(l)}\right), U^{\rho}\left(t_{1}, \ldots, t_{k}, \overrightarrow{\mathcal{V} a r(l)}\right) \rightarrow r\right\}$ if $R$ is normal.

where $x_{1}, \ldots, x_{k}$ are different fresh variables. Note that $\mathbb{U}_{\mathrm{J}}\left(l^{\prime} \rightarrow r^{\prime}\right)=\mathbb{U}_{\mathrm{N}}\left(l^{\prime} \rightarrow r^{\prime}\right)=$ $\left\{l^{\prime} \rightarrow r^{\prime}\right\}$. $\mathbb{U}_{\mathrm{J}}$ and $\mathbb{U}_{\mathrm{N}}$ are extended to join and normal CTRSs, respectively, i.e., $\mathbb{U}_{\mathrm{J}}(R)$ $=\bigcup_{\rho \in R} \mathbb{U}_{\mathrm{J}}(\rho)$ and $\mathbb{U}_{\mathrm{N}}(R)=\bigcup_{\rho \in R} \mathbb{U}_{\mathrm{N}}(\rho)$. We define the extended signatures $\mathcal{F}_{\mathbb{U}_{\mathrm{J}}(R)}$ and $\mathcal{F}_{\mathbb{U}_{\mathrm{N}}(R)}$ of $\mathcal{F}$ as $\mathcal{F}_{\mathbb{U}_{\mathrm{J}}(R)}=\mathcal{F}_{\mathbb{U}_{\mathrm{N}}(R)}=\mathcal{F} \cup\left\{U^{\rho} \mid \rho \in R\right\}$.

Note that $\mathbb{U}_{\mathrm{J}}$ and $\mathbb{U}_{\mathrm{N}}$ are tidy unravelings for join and normal CTRSs, respectively 5 The difference from the original definition in [15] is the replacement of $\overrightarrow{\mathcal{V} a r(r)}$ by $\overrightarrow{\mathcal{V} a r(l)}$. We denote the original unravelings for join and normal CTRSs by $\mathbb{U}_{\mathrm{J}}^{r}$ and $\mathbb{U}_{\mathrm{N}}^{r}$, respectively. $\mathbb{U}_{\mathrm{J}}^{r}$ and $\mathbb{U}_{\mathrm{N}}^{r}$ can be considered optimized variants of $\mathbb{U}_{\mathrm{J}}$ and $\mathbb{U}_{\mathrm{N}}$, respectively, as well as the optimized variant $\mathbb{U}_{\text {opt }}$ of $\mathbb{U}$. The relationship between $\mathbb{U}_{\mathrm{J}}^{r}$ and $\mathbb{U}_{\mathrm{J}}$ and between $\mathbb{U}_{\mathrm{N}}^{r}$ and $\mathbb{U}_{\mathrm{N}}$ is similar to that between $\mathbb{U}_{\text {opt }}$ and $\mathbb{U}$, i.e., if $\mathbb{U}_{\mathrm{J}}^{r}\left(\mathbb{U}_{\mathrm{N}}^{r}\right)$ is sound for a join (normal) CTRS $R$, then so is $\mathbb{U}_{\mathrm{J}}\left(\mathbb{U}_{\mathrm{N}}\right)\left(c f\right.$. ., Corollary [5.5). Thus, in the following, we deal with $\mathbb{U}_{\mathrm{J}}$ and $\mathbb{U}_{\mathrm{N}}$.

Example 5.8. Consider the following join CTRS defining odd and even that, given a natural number $\mathrm{s}^{n}(0)$, return true and false, respectively, if $n$ is odd, and return false and true, respectively, otherwise:

$$
R_{12}=\left\{\begin{array}{cc}
\operatorname{odd}(0) \rightarrow \text { false } & \text { even }(0) \rightarrow \text { true } \\
\operatorname{odd}(\mathrm{s}(x)) \rightarrow \text { true } \Leftarrow \text { even }(x) \downarrow \text { true } & \text { even }(\mathrm{s}(x)) \rightarrow \text { true } \Leftarrow \text { odd }(x) \downarrow \text { true } \\
\text { odd }(\mathrm{s}(x)) \rightarrow \text { false } \Leftarrow \text { even }(x) \downarrow \text { false } & \text { even }(\mathrm{s}(x)) \rightarrow \text { false } \Leftarrow \text { odd }(x) \downarrow \text { false }
\end{array}\right\}
$$

This join CTRS is unraveled by $\mathbb{U}_{\mathrm{J}}$ into the following TRS:

$$
\mathbb{U}_{\mathrm{J}}\left(R_{12}\right)=\left\{\begin{array}{cc}
\vdots & \\
\operatorname{odd}(\mathrm{s}(x)) \rightarrow \mathrm{U}_{22}(\text { even }(x), \text { true }, x) & \mathrm{U}_{22}(y, y, x) \rightarrow \text { true } \\
\operatorname{odd}(\mathrm{s}(x)) \rightarrow \mathrm{U}_{23}(\text { even }(x), \text { false }, x) & \mathrm{U}_{23}(y, y, x) \rightarrow \text { false } \\
\vdots & \\
\text { even }(\mathrm{s}(x)) \rightarrow \mathrm{U}_{24}(\operatorname{odd}(x), \text { true }, x) & \mathrm{U}_{24}(y, y, x) \rightarrow \text { true } \\
\text { even }(\mathrm{s}(x)) \rightarrow \mathrm{U}_{25}(\operatorname{odd}(x), \text { false }, x) & \mathrm{U}_{25}(y, y, x) \rightarrow \text { false }
\end{array}\right\}
$$

\footnotetext{
${ }^{5}$ Compared with sequential unravelings (e.g., $\mathbb{U}$ and $\left.\mathbb{U}_{\mathrm{opt}}\right), \mathbb{U}_{\mathrm{J}}$ and $\mathbb{U}_{\mathrm{N}}$ are called simultaneous unravelings [10].
} 
When we consider $R_{12}$ as a normal CTRS by replacing $\downarrow$ by $\rightarrow$, the CTRS, denoted by $R_{12}^{\prime}$ below, is unraveled by $\mathbb{U}_{\mathrm{N}}$ as follows:

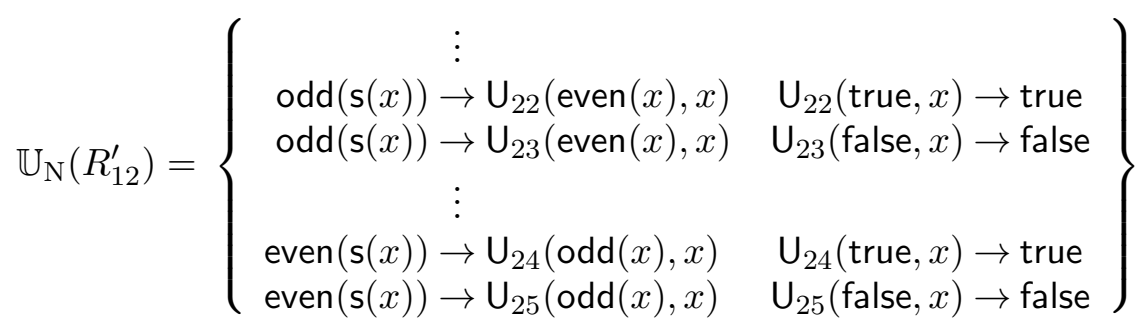

Next, we define a variant of a transformation from join CTRSs to normal ones, that is proposed in [6] ( $c f .$, [26]).

Definition 5.9. Let $R$ be a join CTRS over a signature $\mathcal{F}$. Introducing a fresh binary function symbol eq and a fresh constant $T$, we define a transformation $\mathcal{N}$ orm as follows:

$$
\begin{aligned}
& \operatorname{Norm}\left(l \rightarrow r \Leftarrow s_{1} \downarrow t_{1} ; \ldots ; s_{k} \downarrow t_{k}\right) \\
&=l \rightarrow r \Leftarrow \mathrm{eq}\left(s_{1}, t_{1}\right) \rightarrow \mathrm{eq}(\top, \top) ; \ldots ; \text { eq }\left(s_{k}, t_{k}\right) \rightarrow \mathrm{eq}(\top, \top)
\end{aligned}
$$

and

$$
\mathcal{N} \operatorname{Norm}(R)=\{\operatorname{eq}(x, x) \rightarrow \operatorname{eq}(\top, \top)\} \cup\{\mathcal{N o r m}(\rho) \mid \rho \in R\}
$$

The added rule eq $(x, x) \rightarrow$ eq $(\top, \top)$ results in non-termination, but non-termination does not affect the following discussion $\sqrt{6}$ The difference from the original transformation [6] is the use of eq $(T, T)$ instead of and $T$. The reason of this difference is to make it simple to prove a theorem shown later (Theorem 5.13) - the original transformation, denoted by $n$ in [26], can substitute for $\mathcal{N o r m}$ since $\rightarrow_{n(R)}=\rightarrow_{\mathcal{N o r m}(R)}$ on terms in $\mathcal{T}(\mathcal{F}, \mathcal{V})$. It is clear that $\mathcal{N o r m}(R)$ is a normal CTRS over the signature $\mathcal{F} \cup\{$ eq,$\top\}, \rightarrow_{R}^{*} \subseteq \rightarrow_{\mathcal{N o r m}(R)}^{*}$, and especially $\rightarrow_{R}=\rightarrow_{\mathcal{N o r m}(R)}$ on terms in $\mathcal{T}(\mathcal{F}, \mathcal{V})$. Note that the composed transformation $\mathbb{U}_{\mathrm{N}} \circ \mathcal{N}$ orm is an unraveling for join CTRSs.

Example 5.10. The join CTRS $R_{12}$ in Example 5.8 is transformed by $\mathcal{N}$ orm as follows:

$$
\mathcal{N} \operatorname{Norm}\left(R_{12}\right)=\left\{\begin{array}{c}
\vdots \\
\operatorname{odd}(\mathrm{s}(x)) \rightarrow \text { true } \Leftarrow \text { eq }(\text { even }(x), \text { true }) \rightarrow \text { eq }(\top, \top) \\
\operatorname{odd}(\mathrm{s}(x)) \rightarrow \text { false } \Leftarrow \mathrm{eq}(\text { even }(x), \text { false }) \rightarrow \text { eq }(\top, \top) \\
\vdots \\
\text { even }(\mathrm{s}(x)) \rightarrow \text { true } \Leftarrow \text { eq }(\operatorname{odd}(x), \text { true }) \rightarrow \mathrm{eq}(\top, \top) \\
\text { even }(\mathrm{s}(x)) \rightarrow \text { false } \Leftarrow \text { eq }(\operatorname{odd}(x), \text { false }) \rightarrow \text { eq }(\top, \top) \\
\text { eq }(x, x) \rightarrow \text { eq }(\top, \top)
\end{array}\right\}
$$

\footnotetext{
${ }^{6}$ To avoid non-termination caused by the added rule eq $(x, x) \rightarrow \mathrm{eq}(\top, \top)$, we may introduce a unary constructor $\mathrm{c}_{1}$ as follows:

$$
\begin{aligned}
\mathcal{N o r m}^{\prime}\left(l \rightarrow r \Leftarrow s_{1} \downarrow t_{1} ; \ldots ; s_{k} \downarrow t_{k}\right)= \\
\qquad l \rightarrow r \Leftarrow \mathrm{eq}\left(\mathrm{c}_{1}\left(s_{1}\right), \mathrm{c}_{1}\left(t_{1}\right)\right) \rightarrow \mathrm{eq}(\top, \top) ; \ldots ; \text { eq }\left(\mathrm{c}_{1}\left(s_{k}\right), \mathrm{c}_{1}\left(t_{k}\right)\right) \rightarrow \text { eq }(\top, \top)
\end{aligned}
$$

and

$$
\mathcal{N o r m}^{\prime}(R)=\left\{\operatorname{eq}\left(\mathrm{c}_{1}(x), \mathrm{c}_{1}(x)\right) \rightarrow \operatorname{eq}(\top, \top)\right\} \cup\left\{\mathcal{N o r m}^{\prime}(\rho) \mid \rho \in R\right\}
$$

This variant can substitute for $\mathcal{N}$ orm in the following discussion.
} 
Here, we recall the notion of weak left-linearity. A normal 1-CTRS $R$ is called weakly left-linear (WLL) [9] if any conditional rule with a non-empty condition in $R$ is $\mathbb{U}_{\text {opt }}-\mathrm{LL}$ and any unconditional rule in $R$ is LL w.r.t. non-erasing variables (i.e., for $l \rightarrow r$, the left-hand side is linear w.r.t. variables in $\mathcal{V} a r(l) \cap \mathcal{V} a r(r))$. For example, the normal CTRS $R_{10}$ in Example 4.12 is WLL. $R$ is called ground conditional if, for any rule $l \rightarrow r \Leftarrow$ $s_{1} \approx t_{1} ; \ldots ; s_{k} \approx t_{k}$ in $R$, the terms $s_{1}, t_{1}, \ldots, s_{k}, t_{k}$ in the conditional part are ground. As mentioned before, some soundness conditions for $\mathbb{U}_{\mathrm{N}}$ and $\mathbb{U}_{\mathrm{N}}^{r}$ are known, that are related to the (W)LL property, while no soundness condition for either $\mathbb{U}_{\mathrm{J}}$ or $\mathbb{U}_{\mathrm{J}}^{r}$ is known.

Theorem $5.11([9]) . \mathbb{U}_{\mathrm{N}}$ is sound for WLL normal 1-CTRSs.

The WLL property and Theorem 5.11 lead to the following soundness condition of the composed unraveling $\mathbb{U}_{\mathrm{N}} \circ \mathcal{N}$ orm.

\section{Lemma 5.12.}

- If a join CTRS $R$ is $L L$, then $\mathcal{N o r m}(R)$ is $W L L$.

- $\mathbb{U}_{\mathrm{N}} \circ \mathcal{N}$ orm is sound for LL join CTRSs.

Proof. The first claim is trivial by definition. The second claim follows from the first claim and Theorem 5.11 .

TRSs obtained by $\mathbb{U}_{\mathrm{N}} \circ \mathcal{N}$ orm can completely derive reduction sequences of the corresponding TRSs obtained by $\mathbb{U}_{\mathrm{J}}$, i.e., if $\mathbb{U}_{\mathrm{N}} \circ \mathcal{N}$ orm is sound for a join CTRS $R$, then so is $\mathbb{U}_{\mathrm{J}}$.

Theorem 5.13. Let $R$ be a join $C T R S$ over a signature $\mathcal{F}$. Then, there exists an $\mathcal{F}$-identical and non-erasing tree homomorphism $\phi$ such that $\mathbb{U}_{\mathrm{N}} \circ \mathcal{N}$ orm $(R)=\phi\left(\mathbb{U}_{\mathrm{J}}(R)\right)$. That is, if $\mathbb{U}_{\mathrm{N}} \circ \mathcal{N}$ orm is sound for $R$ (w.r.t. $\underset{\mathrm{evs}}{\mathbb{U}_{\mathrm{N}} \circ \mathcal{N o r m}(R)}$ ), then $\mathbb{U}_{\mathrm{J}}$ is sound for $R$ (w.r.t. $\underset{\mathrm{evs}}{\mathbb{U}_{\mathrm{J}}(R)}$ ).

Proof. Let $\phi$ be an $\mathcal{F}$-identical and non-erasing tree homomorphism determined by $\phi_{\mathcal{F}_{\mathbb{U}_{\mathrm{J}}(R)}}$ such that

$$
\phi_{\mathcal{F}_{\mathbb{U}_{\mathrm{J}}(R)}}\left(U^{\rho}\left(x_{1}, x_{1}^{\prime}, \ldots, x_{k}, x_{k}^{\prime}, \overrightarrow{\mathcal{V} a r(l)}\right)\right)=U^{\rho}\left(\mathrm{eq}\left(x_{1}, x_{1}^{\prime}\right), \ldots, \text { eq }\left(x_{k}, x_{k}^{\prime}\right), \overrightarrow{\mathcal{V} a r(l)}\right)
$$

where $\rho: l \rightarrow r \Leftarrow s_{1} \downarrow t_{1} ; \ldots ; s_{k} \downarrow t_{k} \in R$ and $x_{1}, x_{1}^{\prime}, \ldots, x_{k}, x_{k}^{\prime}$ are fresh different variables. Then, it is clear that $\mathbb{U}_{\mathrm{N}} \circ \mathcal{N} \operatorname{Norm}(R)=\phi\left(\mathbb{U}_{\mathrm{J}}(R)\right)$.

Theorem 5.13 indicates that, for a join CTRS $R$, soundness conditions of $\mathbb{U}_{\mathrm{N}}$ for $\operatorname{Norm}(R)$ are soundness conditions of $\mathbb{U}_{\mathrm{J}}$ for $R$. For example, as a consequence of Lemma 5.12 and Theorem 5.13, we conclude the following result on soundness of $\mathbb{U}_{J}$.

Corollary 5.14. $\mathbb{U}_{\mathrm{J}}$ is sound for LL join 3-CTRSs.

We recognize from Corollary 5.14 that $\mathbb{U}_{\mathrm{J}}$ is sound for $R_{12}$ in Example 5.8,

As we mentioned before, normal CTRSs can be considered join CTRSs because the conditions $s_{i} \rightarrow n_{i}$ and $s_{i} \downarrow n_{i}$ with a ground normal form $n_{i}$ are identical. Thus, soundness of $\mathbb{U}_{\mathrm{J}}$ implies soundness of $\mathbb{U}_{\mathrm{N}}$.

Theorem 5.15. $\mathbb{U}_{\mathrm{N}}$ is sound for a normal CTRS R (w.r.t. $\overrightarrow{\mathrm{evs}}_{\mathbb{U}_{\mathrm{N}}(R)}$ ) if $\mathbb{U}_{\mathrm{J}}$ is sound for the the corresponding join CTRS $R^{\prime}=\left\{l \rightarrow r \Leftarrow s_{1} \downarrow n_{1} ; \ldots ; s_{k} \downarrow n_{k} \mid l \rightarrow r \Leftarrow s_{1} \rightarrow\right.$ $\left.n_{1} ; \ldots ; s_{k} \rightarrow n_{k} \in R\right\}$ (w.r.t. $\overrightarrow{\mathrm{evs}}_{\mathbb{U}_{\mathrm{J}}\left(R^{\prime}\right)}$ ). 
Proof. Let $R$ be over a signature $\mathcal{F}$ and $\phi$ be an $\mathcal{F}$-identical and non-erasing tree homomorphism determined by $\phi_{\mathbb{U}_{\mathrm{N}}(R)}$ such that

$$
\phi_{\mathcal{F}_{\mathbb{U}_{\mathrm{N}}(R)}}\left(U^{\rho}\left(x_{1}, \ldots, x_{k}, \overrightarrow{\mathcal{V} a r(l)}\right)\right)=U^{\rho}\left(x_{1}, n_{1}, \ldots, x_{k}, n_{k}, \overrightarrow{\mathcal{V} a r(l)}\right)
$$

where $\rho: l \rightarrow r \Leftarrow s_{1} \downarrow n_{1} ; \ldots ; s_{k} \downarrow n_{k} \in R$ and $x_{1}, x_{1}^{\prime}, \ldots, x_{k}, x_{k}^{\prime}$ are different fresh variables. Then, it is clear that $\phi\left(\mathbb{U}_{\mathrm{N}}(R)\right)=\mathbb{U}_{\mathrm{J}}\left(R^{\prime}\right)$.

Example 5.16. Consider the join CTRS $R_{12}$ and the corresponding normal CTRS $R_{12}$ in Example 5.8 again. Let $\phi$ be a tree homomorphism determined by the mapping $\phi_{\mathcal{F}}$ such that $\phi_{\mathcal{F}}\left(\mathrm{U}_{22}(y, x)\right)=\mathrm{U}_{22}(y$, true, $x), \phi_{\mathcal{F}}\left(\mathrm{U}_{23}(y, x)\right)=\mathrm{U}_{23}(y$, false, $x), \phi_{\mathcal{F}}\left(\mathrm{U}_{24}(y, x)\right)=$ $\mathrm{U}_{24}(y$, true, $x)$, and $\phi_{\mathcal{F}}\left(\mathrm{U}_{25}(y, x)\right)=\mathrm{U}_{25}(y$, false, $x)$. Then, $\phi\left(\mathbb{U}_{\mathrm{N}}\left(R_{12}^{\prime}\right)\right)=\mathbb{U}_{\mathrm{J}}\left(R_{12}\right)$. Since $\mathbb{U}_{\mathrm{J}}$ is sound for $R_{12}$, we recognize from Theorem 5.15 that $\mathbb{U}_{\mathrm{N}}$ is sound for $R_{12}^{\prime}$.

By the same token, a join CTRS $R$ can be considered a normal CTRS if, for any rule $l \rightarrow r \Leftarrow s_{1} \downarrow t_{1} ; \ldots ; s_{k} \downarrow t_{k} \in R$ and for all $1 \leq i \leq k$, at least one $s_{i}$ and $t_{i}$ is a ground normal form w.r.t. $R_{u}$. We call such a join CTRS $R$ normal and assume w.l.o.g. that $t_{i}$ is a ground normal form w.r.t. $R_{u}$. Then, we obtain the following soundness condition of $\mathbb{U}_{\mathrm{J}}$.

Theorem 5.17. $\mathbb{U}_{\mathrm{J}}$ is sound for a normal join CTRS R (w.r.t. $\overrightarrow{\mathrm{evs}}_{\mathbb{U}_{\mathrm{J}}(R)}$ ) if $\mathbb{U}_{\mathrm{N}}$ is sound for the corresponding normal CTRS $R^{\prime}=\left\{l \rightarrow r \Leftarrow s_{1} \rightarrow n_{1} ; \ldots ; s_{k} \rightarrow n_{k} \mid l \rightarrow r \Leftarrow s_{1} \downarrow\right.$ $\left.n_{1} ; \ldots ; s_{k} \downarrow n_{k} \in R\right\}$ (w.r.t. $\underset{\mathrm{evs}}{\overrightarrow{\mathbb{U}_{\mathrm{N}}}}\left(R^{\prime}\right)$.

Proof. Let $R$ be over a signature $\mathcal{F}$ and $\phi$ be an $\mathcal{F}$-identical and non-erasing tree homomorphism determined by $\phi_{\mathbb{U}_{\mathrm{J}}(R)}$ such that

$$
\phi_{\mathcal{F}_{\mathbb{U}_{\mathrm{J}}(R)}}\left(U^{\rho}\left(x_{1}, x_{1}^{\prime}, \ldots, x_{k}, x_{k}^{\prime}, \overrightarrow{\mathcal{V} a r(l)}\right)\right)=U^{\rho}\left(x_{1}, \ldots, x_{k}, \overrightarrow{\mathcal{V} a r(l)}\right)
$$

where $\rho: l \rightarrow r \Leftarrow s_{1} \downarrow n_{1} ; \ldots ; s_{k} \downarrow n_{k} \in R$ and $x_{1}, x_{1}^{\prime}, \ldots, x_{k}, x_{k}^{\prime}$ are different fresh variables. Then, it is clear that $\phi\left(\mathbb{U}_{\mathrm{J}}(R)\right)=\mathbb{U}_{\mathrm{N}}\left(R^{\prime}\right)$.

It is possible to transform join CTRSs into DCTRSs without adding the rule eq $(x, x) \rightarrow$ eq $(\top, T)$.

Definition 5.18. Let $R$ be a join CTRS over a signature $\mathcal{F}$. Introducing a fresh $2 k$-ary constructor $\mathrm{eq}_{k}$ for each $\rho: l \rightarrow r \Leftarrow s_{1} \downarrow t_{1} ; \ldots ; s_{k} \downarrow t_{k} \in R$, we define a transformation Det as follows:

- $\operatorname{Det}\left(l \rightarrow r \Leftarrow s_{1} \downarrow t_{1} ; \ldots ; s_{k} \downarrow t_{k}\right)=l \rightarrow r \Leftarrow \mathrm{eq}_{k}\left(s_{1}, t_{1}, \ldots, s_{k}, t_{k}\right) \rightarrow \mathrm{eq}_{k}\left(x_{1}, x_{1}, \ldots, x_{k}, x_{k}\right)$ where $x_{1}, \ldots, x_{k}$ are different fresh variables, and

- $\operatorname{Det}(R)=\{\operatorname{Det}(\rho) \mid \rho \in R\}$.

The reason why we introduced $\mathrm{eq}_{k}$ instead of $s_{1} \rightarrow x_{1} ; t_{1} \rightarrow x_{1} ; \ldots ; s_{k} \rightarrow x_{k} ; t_{k} \rightarrow x_{k}$ is to make the number of the conditions in each rule of $\operatorname{Det}(R)$ at most one. It is clear that $\mathbb{U} \circ$ Det is an unraveling for join CTRSs. It is also clear that $\rightarrow_{\mathcal{D e t}(R)}=\rightarrow_{R}$ on terms in $\mathcal{T}(\mathcal{F}, \mathcal{V})$. TRSs obtained by $\mathbb{U} \circ \mathcal{N}$ orm can completely derive reduction sequences of the corresponding TRSs obtained by $\mathbb{U}_{\mathrm{J}}$. This indicates that if $\mathbb{U} \circ \mathcal{D} e t$ is sound for a join CTRS $R$, then so is $\mathbb{U}_{\mathrm{J}}$.

Theorem 5.19. Let $R$ be a join CTRS over a signature $\mathcal{F}$. Then, there exists an $\mathcal{F}$ identical and non-erasing tree homomorphism $\phi$ such that $\mathbb{U} \circ \operatorname{Det}(R)=\phi\left(\mathbb{U}_{\mathrm{J}}(R)\right)$. That is, if $\mathbb{U} \circ$ Det is sound for $R$ (w.r.t. $\overrightarrow{\mathrm{evs}} \mathbb{U} \mathrm{U}$ Det $(R)$ ), then $\mathbb{U}_{\mathrm{J}}$ is sound for $R$ (w.r.t. $\underset{\mathrm{evs}}{\mathbb{U}_{\mathrm{J}}(R)}$ ). 
Proof. Let $\phi$ be an $\mathcal{F}$-identical and non-erasing tree homomorphism determined by $\phi_{\mathcal{F}_{\mathbb{J}^{r}(R)}}$ such that

$$
\phi_{\mathcal{F}_{\mathbb{U}_{\mathrm{J}}(R)}}\left(U^{\rho}\left(x_{1}, x_{1}^{\prime}, \ldots, x_{k}, x_{k}^{\prime}, \overrightarrow{\mathcal{V} a r(l)}\right)\right)=U_{1}^{\rho}\left(\mathrm{eq}_{k}\left(x_{1}, x_{1}^{\prime}, \ldots, x_{k}, x_{k}^{\prime}\right), \overrightarrow{\mathcal{V} a r(l)}\right)
$$

where $\rho: l \rightarrow r \Leftarrow s_{1} \downarrow t_{1} ; \ldots ; s_{k} \downarrow t_{k} \in R$ and $x_{1}, x_{1}^{\prime}, \ldots, x_{k}, x_{k}^{\prime}$ are fresh different variables. Then, it is clear that $\phi\left(\mathbb{U}_{\mathrm{J}}(R)\right)=\mathbb{U} \circ \operatorname{Det}(R)$.

Note that it is easy to adapt Theorem 5.19 to $\mathbb{U}_{N}$ and normal CTRSs.

Normal CTRSs are special cases of DCTRSs, and thus, the unravelings $\mathbb{U}$ and $\mathbb{U}_{\text {opt }}$ for DCTRSs are applicable to normal CTRSs. Moreover, by definition, $\mathbb{U}_{N}$ can be considered a special variant of $\mathbb{U}$ while there is a slight difference: $\mathbb{U}_{N}$ introduces at most one $U$ symbol for each rewrite rule, and $\mathbb{U}$ introduces $k \mathrm{U}$ symbols for each rewrite rule with $k$ conditions. This difference prevents us from using Lemma 5.3 to prove that if $\mathbb{U}_{\mathrm{N}}$ is sound for $R$, then so is $\mathbb{U}$. For this reason, we extend Lemma 5.3 as follows.

Lemma 5.20. Let $U_{1}$ and $U_{2}$ be unravelings, $R$ be an eCTRS over a signature $\mathcal{F}$, and $\mathcal{G}_{1}, \mathcal{G}_{2}$ be extended signatures of $\mathcal{F}$ such that $U_{1}(R)$ and $U_{2}(R)$ are defined over $\mathcal{G}_{1}$ and $\mathcal{G}_{2}$, respectively. Let $\phi$ be an $\mathcal{F}$-identical tree homomorphism determined by $\phi_{\mathcal{G}}$ such that $U_{1}(R)$ $=\phi\left(U_{2}(R)\right) \backslash\left\{t \rightarrow t \mid t \in \mathcal{T}\left(\mathcal{G}_{1}, \mathcal{V}\right) \backslash \mathcal{T}(\mathcal{F}, \mathcal{V})\right\}$. Then, all of the following hold:

$(1) \rightarrow_{U_{2}(R)}^{*} \subseteq \rightarrow_{U_{1}(R)}^{*}$ on terms in $\mathcal{T}(\mathcal{F}, \mathcal{V})$,

(2) if $\phi$ is EV-preserving for $U_{2}(R)$, then $\overrightarrow{\mathrm{evs}}_{U_{2}(R)}^{*} \subseteq \underset{\mathrm{evs}}{U_{1}^{*}(R)}$ on terms in $\mathcal{T}(\mathcal{F}, \mathcal{V})$.

That is, all of the following hold:

(3) if $U_{1}$ is sound for $R$, then so is $U_{2}$, and,

(4) if $\phi$ is EV-preserving for $U_{2}(R)$ and $U_{1}$ is sound for $R$ w.r.t. $\overrightarrow{\mathrm{evs}} U_{1}(R)$, then $U_{2}$ is sound for $R$ w.r.t. $\underset{\mathrm{evs}}{\longrightarrow} U_{2}(R)$.

Proof. We first prove the first claim $\rightarrow_{U_{2}(R)}^{*} \subseteq \rightarrow_{U_{1}(R)}^{*}$ on terms in $\mathcal{T}(\mathcal{F}, \mathcal{V})$. It follows from the assumption that $U_{1}(R)=\left(\phi\left(U_{2}(R)\right) \backslash\left\{t \rightarrow t \mid t \in \mathcal{T}\left(\mathcal{G}_{1}, \mathcal{V}\right)\right\} \cup R^{\prime}\right.$ for some eTRS $R^{\prime}$ $\subseteq\left\{t \rightarrow t \mid t \in \mathcal{T}\left(\mathcal{G}_{1}, \mathcal{V}\right)\right\}$. Then, it follows from Lemma 5.1 that $\phi\left(\rightarrow_{U_{2}(R)}^{*}\right) \subseteq \rightarrow_{U_{1}(R) \cup R^{\prime}}^{*}$ Since $\rightarrow R^{\prime}$ is the identity relation, we have that $\rightarrow_{U_{1}(R) \cup R^{\prime}}^{*}=\rightarrow_{U_{1}(R)}^{*}$, and hence $\phi\left(\rightarrow_{U_{2}(R)}^{*}\right)$ $\subseteq \rightarrow_{U_{1}(R)}^{*}$. Since $\phi$ is $\mathcal{F}$-identical, we have that $\rightarrow_{U_{2}(R)}^{*} \subseteq \rightarrow_{U_{1}(R)}^{*}$ on terms in $\mathcal{T}(\mathcal{F}, \mathcal{V})$.

The second claim follows from the first claim and Lemma 5.2. The third and fourth claims follow from the first and second claims, and soundness of $U_{1}$.

Due to Lemma 5.20, we obtain the following theorem.

Theorem 5.21. Let $R$ be a normal CTRS over a signature $\mathcal{F}$. Then, there exists an $\mathcal{F}$ identical and non-erasing tree homomorphism $\phi$ such that $\mathbb{U}_{\mathrm{N}}(R)=\phi(\mathbb{U}(R)) \backslash\{t \rightarrow t \mid t \in$ $\left.\mathcal{T}\left(\mathcal{F}_{\mathbb{U}(R)}, \mathcal{V}\right) \backslash \mathcal{T}(\mathcal{F}, \mathcal{V})\right\}$. That is, if $\mathbb{U}_{\mathrm{N}}$ is sound for a normal CTRS R (w.r.t. $\left.\overrightarrow{\mathrm{evs}}_{\mathbb{U}_{\mathrm{N}}(R)}\right)$, then $\mathbb{U}$ is sound for $R$ (w.r.t. $\underset{\mathrm{evs}}{\longrightarrow} \mathbb{U}(R)$ ).

Proof. Let $\phi$ be an $\mathcal{F}$-identical tree homomorphism determined by $\phi_{\mathcal{F}_{\mathbb{U}(R)}}$ such that

$$
\phi_{\mathcal{F}_{\mathbb{U}(R)}}\left(U_{i}^{\rho}\left(x_{i}, \vec{X}_{i}\right)\right)=U^{\rho}\left(n_{1}, \ldots, n_{i-1}, x_{i}, s_{i+1}, \ldots, s_{k}, \overrightarrow{\mathcal{V} a r(l)}\right)
$$

where $\rho: l \rightarrow r \Leftarrow s_{1} \rightarrow n_{1} ; \ldots ; s_{k} \rightarrow n_{k} \in R$ and $x_{i}$ is a fresh variable. It is clear that $\mathbb{U}_{\mathrm{N}}(R)=\phi(\mathbb{U}(R)) \backslash\left\{t \rightarrow t \mid t \in \mathcal{T}\left(\mathcal{F}_{\mathbb{U}(R)}, \mathcal{V}\right) \backslash \mathcal{T}(\mathcal{F}, \mathcal{V})\right\}:$

- $\phi\left(l \rightarrow U_{1}^{\rho}\left(s_{1}, \overrightarrow{X_{1}}\right)\right)=l \rightarrow U^{\rho}\left(s_{1}, \ldots, s_{k}, \overrightarrow{\mathcal{V} a r(l)}\right) \in \mathbb{U}_{\mathrm{N}}(R)$, 
- $\phi\left(U_{i}^{\rho}\left(n_{i}, \vec{X}_{i}\right) \rightarrow U_{i+1}^{\rho}\left(s_{i+1}, \overrightarrow{X_{i+2}}\right)\right)=U^{\rho}\left(n_{1}, \ldots, n_{i}, s_{i+1}, \ldots, s_{k}, \overrightarrow{\mathcal{V} a r}(l)\right) \rightarrow U^{\rho}\left(n_{1}, \ldots, n_{i}\right.$, $\left.s_{i+1}, \ldots, s_{k}, \overrightarrow{\mathcal{V} a r(l)}\right) \in\left\{t \rightarrow t \mid t \in \mathcal{T}\left(\mathcal{F}_{\mathbb{U}_{\mathrm{N}}(R)}, \mathcal{V}\right) \backslash \mathcal{T}(\mathcal{F}, \mathcal{V})\right\}$, and

- $\phi\left(U_{k}^{\rho}\left(n_{k}, \overrightarrow{X_{k}}\right) \rightarrow r\right)=U^{\rho}\left(n_{1}, \ldots, n_{k}, \overrightarrow{\mathcal{V} a r(l)}\right) \rightarrow r \in \mathbb{U}_{\mathrm{N}}(R)$.

Since $R$ is normal, we have that $\mathcal{V}$ ar $\left(n_{1}, \ldots, n_{k}\right)=\emptyset$ and $\mathcal{V} a r\left(s_{1}, \ldots, s_{k}\right) \subseteq \mathcal{V}$ ar $(l)$, and hence $X_{i}=\mathcal{V} a r(l)$ for all $1 \leq i \leq k$. Thus, $\operatorname{Var}\left(\phi_{\mathcal{F}_{\mathbb{U}(R)}}\left(U_{i}^{\rho}\left(x_{i}, \vec{X}_{i}\right)\right)\right)=\left\{x_{i}\right\} \cup X_{i}=\left\{x_{i}\right\} \cup \mathcal{V} a r(l)$ $=\operatorname{Var}\left(U^{\rho}\left(n_{1}, \ldots, n_{i-1}, x_{i}, s_{i+1}, \ldots, s_{k}, \overrightarrow{\mathcal{V} a r(l)}\right)\right)$, and hence $\phi$ is non-erasing.

It is not known whether the converse of Theorem $\left[5.21\right.$ (i.e., $\rightarrow_{\mathbb{U}_{\mathrm{N}}(R)}^{*} \subseteq \rightarrow_{\mathbb{U}(R)}^{*}$ on terms in $\mathcal{T}(\mathcal{F}, \mathcal{V})$ ) holds or not. In other words, it is not known whether the following claim holds or not: if $\mathbb{U}$ is sound for a normal CTRS, then so is $\mathbb{U}_{\mathrm{N}}$. As we mentioned before, to show soundness of $\mathbb{U}_{\mathrm{N}}$ by means of $\mathbb{U}$, we would like to show that, for any normal CTRS $R$, all the derivations of $\mathbb{U}_{\mathrm{N}}(R)$ can be derived by $\mathbb{U}(R)$. However, this is not true in general.

Example 5.22. Consider the following variant $R_{3}^{\prime}$ of the DCTRS $R_{3}$ in Example 3.3, that is obtained by replacing the conditional part $x \rightarrow \mathrm{e}$ of the first rule by $x \rightarrow \mathrm{e} ; x \rightarrow \mathrm{e}^{\prime}$ and by adding $\mathrm{c} \rightarrow \mathrm{e}^{\prime}$ to the rules:

$$
R_{3}^{\prime}=\left\{\begin{aligned}
\mathrm{f}(x) & \rightarrow x \Leftarrow x \rightarrow \mathrm{e} ; x \rightarrow \mathrm{e}^{\prime} \\
\mathrm{g}(\mathrm{d}, x, x) & \rightarrow \mathrm{A} \\
\mathrm{h}(x, x) & \rightarrow \mathrm{g}(x, x, \mathrm{f}(\mathrm{k})) \\
\mathrm{c} & \rightarrow \mathrm{e}^{\prime}
\end{aligned}\right\} \cup R_{0}
$$

The CTRS $R_{3}^{\prime}$ is unraveled by $\mathbb{U}_{\mathrm{N}}$ and $\mathbb{U}$ as follows:

$$
\mathbb{U}_{\mathrm{N}}\left(R_{3}^{\prime}\right)=\left\{\begin{array}{c}
\mathrm{f}(x) \rightarrow \mathrm{U}_{4}(x, x, x) \\
\mathrm{U}_{4}\left(\mathrm{e}, \mathrm{e}^{\prime}, x\right) \rightarrow x \\
\vdots
\end{array}\right\} \cup R_{0} \quad \mathbb{U}\left(R_{3}^{\prime}\right)=\left\{\begin{array}{c}
\mathrm{f}(x) \rightarrow \mathrm{U}_{4}^{\prime}(x, x) \\
\mathrm{U}_{4}^{\prime}(\mathrm{e}, x) \rightarrow \mathrm{U}_{4}^{\prime \prime}(x, x) \\
\mathrm{U}_{4}^{\prime \prime}\left(\mathrm{e}^{\prime}, x\right) \rightarrow x \\
\vdots
\end{array}\right\} \cup R_{0}
$$

We have that $\mathrm{h}(\mathrm{f}(\mathrm{a}), \mathrm{f}(\mathrm{b})) \rightarrow_{\mathbb{U}_{\mathrm{N}}\left(R_{3}^{\prime}\right)}^{*} \mathrm{~A}$, but $\mathrm{h}(\mathrm{f}(\mathrm{a}), \mathrm{f}(\mathrm{b})) t_{\mathbb{U}\left(R_{3}^{\prime}\right)}^{*}$ A. This means that $\mathbb{U}\left(R_{3}^{\prime}\right)$ cannot derive every reduction sequence of $\mathbb{U}_{\mathrm{N}}\left(R_{3}^{\prime}\right)$ that starts from terms over the original signature of $R_{3}^{\prime}$. U seems sound for $R_{3}^{\prime}$. However, we have no sufficient condition to prove soundness of $\mathbb{U}$ for $R_{3}^{\prime}$, and thus, it is not known whether $\mathbb{U}$ is sound for $R_{3}^{\prime}$ or not.

The symbols e and $e^{\prime}$ are used for the same role; therefore, this distinction is meaningless. Thus, the replacement of $x \rightarrow \mathrm{e}$ with $x \rightarrow \mathrm{e} ; x \rightarrow \mathrm{e}$ is sufficient for the purpose of this example. For the original CTRS $R_{3}^{\prime}$, this duplication of $x \rightarrow \mathrm{e}$ is quite meaningless, but this greatly affects the reduction of $\mathbb{U}\left(R_{3}^{\prime}\right)$. For this reason, this would be an interesting example for investigating soundness conditions of unravelings.

A trivial sufficient condition for the converse of Theorem 5.21 is that any rule of $R$ has at most one condition: by considering $U^{\rho}=U_{1}^{\rho}$, we have that $\mathbb{U}_{\mathrm{N}}(R)=\mathbb{U}(R)$.

As stated above, the relationship between $\mathbb{U}_{\mathrm{J}}^{r}, \mathbb{U}_{\mathrm{N}}^{r}$, and $\mathbb{U}_{\text {opt }}$ is similar to that between $\mathbb{U}_{\mathrm{J}}, \mathbb{U}_{\mathrm{N}}$, and $\mathbb{U}$. For this reason, Theorems 5.15, 5.17, 5.21 also hold for $\mathbb{U}_{\mathrm{J}}^{r}, \mathbb{U}_{\mathrm{N}}^{r}$, and $\mathbb{U}_{\text {opt }}$.

\section{Comparison with Şerbănuţă-Roşu Transformation}

In this section, we compare the unraveling $\mathbb{U}$ with the SR transformation, in terms of soundness, operational termination, confluence, computational equivalence, and so on. 
6.1. Formalization of Transformations for CTRSs. In this subsection, to make it easier to compare unravelings with other transformations, we first formalize transformations of CTRSs into TRSs, and also generalize the notion of soundness and completeness for unravelings. Then, we present relationship between soundness of two transformations by generalizing Lemma 5.3 ,

We first formalize transformations of CTRSs and the notions of soundness and completeness.

Definition 6.1 (CTRS transformations). A CTRS transformation is a computable transformation $T$ from eCTRSs into eTRSs with injective mappings as follows: for an eCTRS $R$ over a signature $\mathcal{F}$, the transformed eTRS $R_{T}$ over a signature $\mathcal{G}$ is defined and the corresponding mapping $\phi_{T(R)}$ from $\mathcal{T}(\mathcal{F}, \mathcal{V})$ to $\mathcal{T}(\mathcal{G}, \mathcal{V})$ is also defined, i.e., $T(R)=\left(R_{T}, \phi_{T(R)}\right)$. The mapping $\phi_{T(R)}$ is called a translation related to $T(R)$ We extend $\phi_{T(R)}$ to pairs of terms in $\mathcal{T}(\mathcal{F}, \mathcal{V})$ : for $S \subseteq \mathcal{T}(\mathcal{F}, \mathcal{V}) \times \mathcal{T}(\mathcal{F}, \mathcal{V}), \phi(S)=\{(\phi(s), \phi(t)) \mid(s, t) \in S\}$. Moreover, $T$ is called simple if the related translation $\phi_{T(R)}$ is the identity mapping (i.e., $\mathcal{F} \subseteq \mathcal{G}$ and $\phi_{T(R)}(t)=t$ for all $\left.t \in \mathcal{T}(\mathcal{F}, \mathcal{V})\right)$, and we abuse notation and write $T(R)$ as the transformed system $R_{T}$.

Let $\Rightarrow_{R_{T}}$ be a subrelation of $\rightarrow_{R_{T}}$.

- $T$ is called sound for $R$ w.r.t. $\Rightarrow_{R_{T}}$ if $\Rightarrow_{R_{T}}^{*} \subseteq \phi_{T(R)}\left(\rightarrow_{R}^{*}\right)$ on terms in $\mathcal{T}(\mathcal{F}, \mathcal{V})$ (i.e., for all terms $s, t$ in $\mathcal{T}(\mathcal{F}, \mathcal{V})$, if $\phi_{T(R)}(s) \Rightarrow_{R_{T}}^{*} \phi_{T(R)}(t)$, then $\left.s \rightarrow_{R}^{*} t\right)$.

- $T$ is called complete for $R$ w.r.t. $\Rightarrow_{R_{T}}$ if $\phi_{T(R)}\left(\rightarrow_{R}^{*}\right) \subseteq \Rightarrow_{R_{T}}^{*}$ (i.e., for all terms $s, t \in$ $\mathcal{T}(\mathcal{F}, \mathcal{V})$, if $s \rightarrow_{R}^{*} t$, then $\left.\phi_{T(R)}(s) \Rightarrow_{R_{T}}^{*} \phi_{T(R)}(t)\right)$.

When $T$ is sound and complete for $R$ w.r.t. $\rightarrow_{R_{T}}$, we simply say that $T$ is sound and complete for $R$, respectively. Moreover, $T$ is called sound (complete) if $T$ is sound (complete) for any eCTRS $R$ such that $T(R)$ is defined.

Note that unravelings are complete simple CTRS transformations.

Next, we generalize Lemma 5.3 to two CTRS transformations, one of which is simple.

Theorem 6.2. Let $T$ be a CTRS transformation, $U$ be a simple CTRS transformation, $R$ be an eCTRS over a signature $\mathcal{F}$ such that $T(R)$ and $U(R)$ are defined, $R_{T}$ is over a signature $\mathcal{G}_{T}$, and $U(R)$ is over a signature $\mathcal{G}_{U}$. Then, all of the following hold:

- if $T$ is sound for $R$ and $\phi\left(\rightarrow_{U(R)}^{*}\right) \subseteq \rightarrow_{R_{T}}^{*}$, 8 then $U$ is sound for $R$,

- if $U$ is sound for $R$ and $\rightarrow_{R_{T}}^{*} \subseteq \phi\left(\rightarrow_{U(R)}^{*}\right)$, then $T$ is sound for $R$,

- if $T$ is complete for $R$ and $\rightarrow_{R_{T}}^{*} \subseteq \phi\left(\rightarrow_{U(R)}^{*}\right)$, then $U$ is complete for $R$, and

- if $U$ is complete for $R$ and $\phi\left(\rightarrow_{U(R)}^{*}\right) \subseteq \rightarrow_{R_{T}}^{*}$, then $T$ is complete for $R$.

Proof. We only prove the first claim since the other claims can be proved similarly to the first one. Let $s, t$ be terms in $\mathcal{T}(\mathcal{F}, \mathcal{V})$. Suppose that $s \rightarrow_{U(R)}^{*} t$. Then, it follows from $\phi\left(\rightarrow_{U(R)}^{*}\right) \subseteq \rightarrow_{R_{T}}^{*}$ that $\phi(s) \rightarrow_{R_{T}}^{*} \phi(t)$. It follows from soundness of $T$ for $R$ that $s \rightarrow_{R}^{*} t$, and hence $U$ is sound for $R$.

7 The mapping $\phi$ can be considered a translation from original terms for $R$ into the corresponding ones for $T(R)$.

8 Note that $\phi\left(\rightarrow_{U(R)}^{*}\right)=\left\{(\phi(s), \phi(t)) \mid s, t \in \mathcal{T}(\mathcal{F}, \mathcal{V}), s \rightarrow_{U(R)}^{*} t\right\}$ since $\phi$ is not defined for any term containing a function symbol in $\mathcal{G}_{U} \backslash \mathcal{F}$. 
6.2. Şerbănuţă-Roşu Transformation. In this subsection, we recall the definition of the SR transformation proposed by Şerbănuţă and Roşu [30, 31], which is basically applied to strongly or syntactically DCTRSs. We also recall some of its properties.

Let $R$ be an eDCTRS. A term $t$ is called strongly irreducible w.r.t. $R$ if $t \sigma$ is a normal form w.r.t. $R$ for every normalized substitution $\sigma$. $R$ is called strongly deterministic (strongly DCTRS) if, for every rule $l \rightarrow r \Leftarrow s_{1} \rightarrow t_{1} ; \ldots ; s_{k} \rightarrow t_{k} \in R$, every term $t_{i}$ is strongly irreducible w.r.t. $R$. $R$ is called syntactically deterministic (syntactically DCTRS) if, for every rule $l \rightarrow r \Leftarrow s_{1} \rightarrow t_{1} ; \ldots ; s_{k} \rightarrow t_{k} \in R$, every term $t_{i}$ is a constructor term or a ground normal form w.r.t. $R_{u}$. Note that normal CTRSs are syntactically DCTRSs, and syntactically DCTRSs are also strongly DCTRSs.

In the following, we assume that for each defined symbol $f$ of $R$, there are $n_{f}$ many $f$ rules in $R$ that have non-empty conditions and are ordered. We denote the $i$-th conditional rewrite rule of $f$ with a non-empty condition by $\rho_{f, i}$.

In the SR transformation $\mathbb{S R}$ below, a fresh unary function symbol $\{\cdot\}$, a fresh constant $\perp$, and fresh $k$-ary constructors $[\cdot]_{k}$ are introduced and for a defined symbol $f$, a fresh function symbol $\bar{f}$ is introduced by adding $n_{f}$ arguments to $f$. The " $n+i$ "-th argument of $\bar{f}$ is used for evaluating the conditions of the $i$-th conditional rule $\rho_{f, i}: l \rightarrow$ $r \Leftarrow s_{1} \rightarrow t_{1} ; \ldots ; s_{k} \rightarrow t_{k}$, by initializing with $\perp$ and by replacing $\perp$ with an instance of $\left[\left\{\overline{s_{1}}\right\}, \perp, \ldots, \perp\right]_{k}$ to start the evaluation, where $\overline{s_{1}}$ is the term obtained by replacing each defined symbol $f$ by $\bar{f}$ with filling extra arguments with $\perp$. The $k$-ary symbol $[\cdot]_{k}$ is used as a stack with $k$ elements, e.g., when $\left[\left\{\overline{s_{1}} \sigma\right\}, \perp, \ldots, \perp\right]_{k}$ is reduced to $\left[\left\{\overline{t_{1}} \theta\right\}, \perp, \ldots, \perp\right]_{k}$, the evaluation of the second condition $s_{2} \rightarrow t_{2}$ with $\theta$ starts from $\left[\left\{\overline{s_{2}} \theta\right\}, \overline{t_{1}} \theta, \perp, \ldots, \perp\right]_{k}$.

Definition 6.3 (SR transformation $\mathbb{S R}[31]$ ). Let $R$ be a strongly or syntactically DCTRS over a signature $\mathcal{F}^{9}$ For $f \in \mathcal{D}_{R}$, we prepare a function symbol $\bar{f}$ with $\operatorname{arity}(\bar{f})=$ $\operatorname{arity}(f)+n_{f}$. Introducing a fresh unary function symbol $\{\cdot\}$, a fresh constant $\perp$ and fresh $j$-ary constructors $[\cdot]_{j}$ with $j>0\left([\cdot]_{1},[\cdot]_{2}, \ldots\right.$ are sometimes abbreviated to $\left.[\cdot]\right)$ into the signature, the DCTRS $R$ is transformed into the following TRS $\mathbb{S R}^{\rightarrow}(R)$ :

$$
\begin{aligned}
& \mathbb{S R}^{\text {rule }}\left(\rho_{f, i}: f\left(w_{1}, \ldots, w_{n}\right) \rightarrow r \Leftarrow s_{1} \rightarrow t_{1} ; \ldots ; s_{k} \rightarrow t_{k}\right)= \\
& \left\{\begin{array}{c}
\bar{f}\left(\overline{w_{1}}, \ldots, \overline{w_{n}}, z_{1}, \ldots, z_{i-1}, \perp, z_{i+1}, \ldots, z_{n_{f}}\right) \\
\rightarrow \bar{f}\left(\overline{w_{1}}, \ldots, \overline{w_{n}}, z_{1}, \ldots, z_{i-1},\left[\left\{\overline{s_{1}}\right\}, \perp, \ldots, \perp\right]_{k}, z_{i+1}, \ldots, z_{n_{f}}\right) \\
\bar{f}\left(\overline{w_{1}}, \ldots, \overline{w_{n}}, z_{1}, \ldots, z_{i-1},\left[\left\{\overline{t_{1}}\right\}, \perp, \ldots, \perp\right]_{k}, z_{i+1}, \ldots, z_{n_{f}}\right) \\
\rightarrow \bar{f}\left(\overline{w_{1}}, \ldots, \overline{w_{n}}, z_{1}, \ldots, z_{i-1},\left[\left\{\overline{s_{2}}\right\}, \overline{t_{1}}, \ldots, \perp\right]_{k}, z_{i+1}, \ldots, z_{n_{f}}\right) \\
\vdots \\
\bar{f}\left(\overline{w_{1}}, \ldots, \overline{w_{n}}, z_{1}, \ldots, z_{i-1},\left[\left\{\overline{t_{k-1}}\right\}, \overline{t_{k-2}}, \ldots, \overline{t_{1}}, \perp\right]_{k}, z_{i+1}, \ldots, z_{n_{f}}\right) \\
\rightarrow \bar{f}\left(\overline{w_{1}}, \ldots, \overline{w_{n}}, z_{1}, \ldots, z_{i-1},\left[\left\{\overline{s_{k}}\right\}, \overline{t_{k-1}}, \ldots, \overline{t_{1}}\right]_{k}, z_{i+1}, \ldots, z_{n_{f}}\right) \\
\bar{f}\left(\overline{w_{1}}, \ldots, \overline{w_{n}}, z_{1}, \ldots, z_{i-1},\left[\left\{\overline{t_{k}}\right\}, \overline{t_{k-1}}, \ldots, \overline{t_{1}}\right]_{k}, z_{i+1}, \ldots, z_{n_{f}}\right) \rightarrow\{\bar{r}\}
\end{array}\right\}
\end{aligned}
$$

where $z_{1}, \ldots, z_{n_{f}}$ are fresh different variables and the operation $₹$ is a linear non-erasing tree homomorphism determined by $\bar{\phi}$ such that

- $\bar{\phi}\left(c\left(x_{1}, \ldots, x_{n}\right)=c\left(x_{1}, \ldots, x_{n}\right)\right.$ for an $n$-ary constructor $c \in \mathcal{C}_{R}$, and

\footnotetext{
${ }^{9}$ In 31, it is assumed that any deterministic conditional rule $l \rightarrow r \Leftarrow s_{1} \rightarrow t_{1} ; \ldots ; s_{k} \rightarrow t_{k}$ satisfies $\mathcal{V} a r\left(s_{i}\right) \nsubseteq \mathcal{V} a r\left(l, t_{1}, \ldots, t_{i-2}\right)$, i.e., the $i$-th condition $s_{i} \rightarrow t_{i}$ cannot be evaluated before finishing the evaluation of the " $i-1$ "-th condition $s_{i-1} \rightarrow t_{i-1}$. However, this is not essential for the definition of $\mathbb{S R}$.
} 
- $\bar{\phi}\left(f\left(x_{1}, \ldots, x_{n}\right)=\bar{f}(x_{1}, \ldots, x_{n}, \overbrace{\perp, \ldots, \perp}^{n_{f}})\right.$ for an $n$-ary defined symbol $f \in \mathcal{D}_{R}$.

Note that the operation ${ }^{-}$is injective. The transformed $\operatorname{TRS} \mathbb{S R}^{\rightarrow}(R)$ is defined as follows:

$$
\begin{aligned}
& \mathbb{S R}^{\rightarrow}(R)=\bigcup_{\rho \in R} \mathbb{S R}^{\text {rule }}(\rho) \\
& \cup\left\{\bar{f}\left(x_{1}, \ldots, x_{i-1},\left\{x_{i}\right\}, x_{i+1}, \ldots, x_{n}, z_{1}, \ldots, z_{n_{f}}\right) \rightarrow\left\{\bar{f}\left(x_{1}, \ldots, x_{n}, \perp, \ldots, \perp\right)\right\} \mid f \in \mathcal{D}_{R}\right\} \\
& \cup\left\{c\left(x_{1}, \ldots, x_{i-1},\left\{x_{i}\right\}, x_{i+1}, \ldots, x_{n}\right) \rightarrow\left\{c\left(x_{1}, \ldots, x_{n}\right)\right\} \mid c \in \mathcal{C}_{R}\right\} \\
& \cup\{\{\{x\}\} \rightarrow\{x\}\}
\end{aligned}
$$

where $x_{1}, \ldots, x_{n}, z_{1}, \ldots, z_{n_{f}}$ are variables. Note that $\mathbb{S R}_{R}(R)$ is a TRS over $\mathcal{F}_{\mathbb{S R}(R)}=$ $\{\perp,\{\cdot\}\} \cup\left\{\bar{f} \mid f \in \mathcal{D}_{R}\right\} \cup \mathcal{C}_{R}$. Moreover, a partial mapping $\widehat{\cdot}$ from $\mathcal{T}\left(\mathcal{F}_{\mathbb{S R}(R)}, \mathcal{V}\right)$ to $\mathcal{T}(\mathcal{F}, \mathcal{V})$ is defined as follows:

- $\widehat{x}=x$ for $x \in \mathcal{V}$,

- $\widehat{\{t\}}=\widehat{t}$,

- $c\left(\widehat{t_{1}, \ldots,}, t_{n}\right)=c\left(\widehat{t_{1}}, \ldots, \widehat{t_{n}}\right)$ for an $n$-ary constructor $c \in \mathcal{C}_{R}$, and

- $\bar{f}\left(t_{1}, \ldots, \widehat{t_{n}, u_{1}}, \ldots, u_{n_{f}}\right)=f\left(\widehat{t_{1}}, \ldots, \widehat{t_{n}}\right)$ for an $n$-ary defined symbol $f \in \mathcal{D}_{R}$.

Note that the operation $\hat{\cdot}$ partially translates terms in $\mathcal{T}\left(\mathcal{F}_{\mathbb{S R}(R)}, \mathcal{V}\right)$ back into terms in $\mathcal{T}(\mathcal{F}, \mathcal{V})$. The $S R$ transformation $\mathbb{S R}$ is defined as $\mathbb{S R}(R)=\left(\mathbb{S R}^{\rightarrow}(R), \phi_{\mathbb{S R}(R)}\right)$, where the translation $\phi_{\mathbb{S R}(R)}$ is defined as $\phi_{\mathbb{S R}(R)}(t)=\{\bar{t}\}$. Moreover, a term $t$ in $\mathcal{T}\left(\mathcal{F}_{\mathbb{S R}(R)}, \mathcal{V}\right)$ is called reachable if there exists a term $s$ in $\mathcal{T}(\mathcal{F}, \mathcal{V})$ such that $\phi_{\mathbb{S R}(R)}(s) \rightarrow_{\mathbb{S R}^{*}(R)}^{*}\{t\}$.

Note that $\mathbb{S R}$ is a complete CTRS transformation [31]. By definition, it is clear that $R$ is $\mathbb{U}_{\text {opt }}-L L$ iff $\mathbb{S R}^{\rightarrow}(R)$ is LL. A reachable term $s$ has the following property:

- every subterm of $s$, rooted by $\bar{f}$, is of the form $\bar{f}\left(s_{1}, \ldots, s_{n}, u_{1}, \ldots, u_{n_{f}}\right)$ such that, for all $1 \leq j \leq n_{f}, u_{j}$ is either $\perp$ or of the form $\left[\left\{\overline{t_{i}}\right\}, \overline{t_{i-1}}, \ldots, \overline{t_{1}}, \perp, \ldots, \perp\right]_{k}$ for some $i$, where $\rho_{f, j}: f\left(w_{1}, \ldots, w_{n}\right) \rightarrow r \Leftarrow s_{1} \rightarrow t_{1} ; \ldots ; s_{k} \rightarrow t_{k} \in R$, and

- both the symbols $\perp$ and $[\cdot]_{k}$ appear only as in the form mentioned in the previous case.

For a DCTRS $R$, the transformed TRS $\mathbb{S R}^{\rightarrow}(R)$ is overlapping (not only at root position, but also at properly inner positions), thus not a constructor system, and all nonconstant constructors of $R$ are defined symbols of $\mathbb{S R}^{\rightarrow}(R)$. However, critical pairs generated from rules to push out the special constructor $\{\cdot\}$ are joinable and they are not so critical in terms of confluence. 
Example 6.4 ([31]). Consider the $\mathbb{U}_{\text {opt }}$-LL DCTRS $R_{7}$ in Example 4.4 again. $R_{7}$ is a syntactically DCTRS and it is transformed by $\mathbb{S R}^{\rightarrow}$ into the following TRS:

$$
\begin{aligned}
& \mathbb{S R}^{\rightarrow}\left(R_{7}\right)=
\end{aligned}
$$

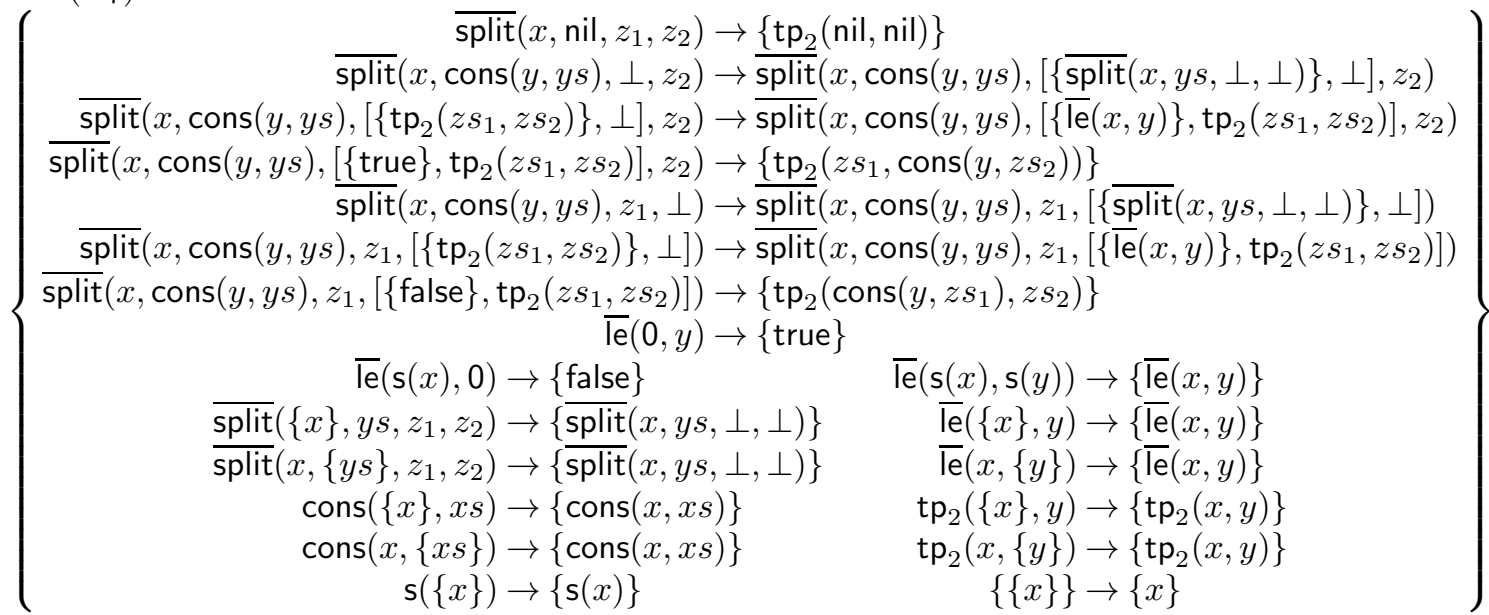

Consider the term split(s(0), cons $(0, \operatorname{cons}(\mathrm{s}(\mathrm{s}(0))$, nil $)))$. Starting from its translated term, we have the following derivation of $\mathbb{S R}^{\rightarrow}\left(R_{7}\right)$ under the leftmost innermost strategy that selects the topmost rules of applicable ones:

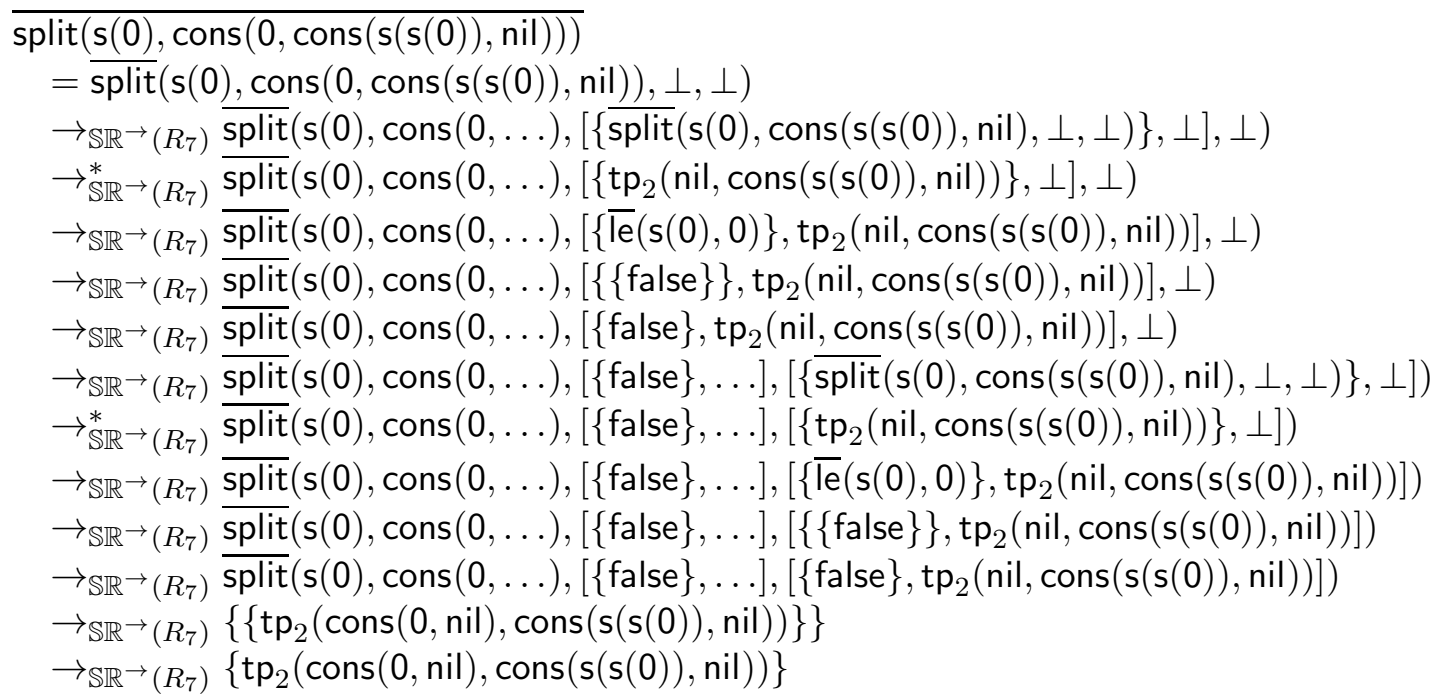

By applying the translation-back mapping $\widehat{\cdot}$ to $\left\{\operatorname{tp}_{2}(\operatorname{cons}(0\right.$, nil $)$, cons $(\mathrm{s}(\mathrm{s}(0))$, nil $\left.))\right\}$, we obtain $\operatorname{tp}_{2}(\operatorname{cons}(0$, nil $), \operatorname{cons}(\mathrm{s}(\mathrm{s}(0))$, nil $))$, a normal form of $\operatorname{split}(\mathrm{s}(0), \operatorname{cons}(0, \operatorname{cons}(\mathrm{s}(\mathrm{s}(0))$, nil $)))$ w.r.t. $R_{7}$.

The SR transformation $\mathbb{S R}$ has the following properties.

Theorem 6.5 ([31]). Let $R$ be a strongly or syntactically DCTRS. Then, all of the following hold:

- $\mathbb{S R}$ is sound for $R$ if $R$ is confluent 10 or $\mathbb{U}_{\text {opt }}-L L$,

- if $R$ is $\mathbb{U}_{\mathrm{opt}}-L L$ and $\mathbb{S R}^{\rightarrow}(R)$ is confluent on reachable terms, then $R$ is confluent, and

\footnotetext{
10 In 30, 31, soundness and completeness are discussed on ground reduction sequences only. In the proof of soundness and completeness, groundness of terms in derivations is only used with groundness in
} 
- if $R$ is $\mathbb{U}_{\mathrm{opt}}-L L$ and confluent, then $\mathbb{S R}^{\rightarrow}(R)$ is confluent on reachable terms.

We recognize from the second statement of Theorem 6.5 that confluence of $\mathbb{S R}^{\rightarrow}(R)$ is a sufficient condition for confluence of $R$.

Example 6.6. Consider the DCTRS $R_{7}$ and the transformed TRS $\operatorname{SR}\left(R_{7}\right)$ in Examples 4.4, 6.4 again. The DCTRS $R_{7}$ is operationally terminating since $\mathbb{S R}^{\rightarrow}\left(R_{7}\right)$ is terminating [31]. We have only a critical pair of $R_{7}$ between the second and third rules. The critical pair is infeasible since there exists no terms $s, t$ such that le $(s, t) \rightarrow_{R_{7}}^{*}$ true and le $(s, t) \rightarrow_{R_{7}}^{*}$ false. Thus, we can see that $R_{7}$ is confluent [2] (cf., [26]). Though, we have no formal method for proving confluence of $R_{7}$. On the other hand, all the critical pairs of $\mathbb{S R}\left(R_{7}\right)$ are joinable and $\mathbb{S R}^{\rightarrow}\left(R_{7}\right)$ is terminating, and hence $\mathbb{S R}^{\rightarrow}\left(R_{7}\right)$ is confluent. Due to Theorem [6.5, confluence of $\mathbb{S R}^{\rightarrow}\left(R_{7}\right)$ guarantees confluence of $R_{7}$.

Consider the unraveled TRS $\mathbb{U}\left(R_{7}\right)$ :

$$
\mathbb{U}\left(R_{7}\right)=\left\{\begin{array}{c}
\vdots \\
\operatorname{split}(x, \operatorname{cons}(y, y s)) \rightarrow \mathrm{U}_{13}(\operatorname{split}(x, y s), x, y, y s) \\
\mathrm{U}_{13}\left(\operatorname{tp}_{2}\left(z s_{1}, z s_{2}\right), x, y, y s\right) \rightarrow \mathrm{U}_{14}\left(\operatorname{le}(x, y), x, y, y s, z s_{1}, z s_{2}\right) \\
\mathrm{U}_{14}\left(\operatorname{true}, x, y, y s, z s_{1}, z s_{2}\right) \rightarrow \operatorname{tp}_{2}\left(z s_{1}, \operatorname{cons}\left(y, z s_{2}\right)\right) \\
\operatorname{split}(x, \operatorname{cons}(y, y s)) \rightarrow \mathrm{U}_{15}(\operatorname{split}(x, y s), x, y, y s) \\
\mathrm{U}_{15}\left(\operatorname{tp}_{2}\left(z s_{1}, z s_{2}\right), x, y, y s\right) \rightarrow \mathrm{U}_{16}\left(\operatorname{le}(x, y), x, y, y s, z s_{1}, z s_{2}\right) \\
\mathrm{U}_{16}\left(\text { false }, x, y, y s, z s_{1}, z s_{2}\right) \rightarrow \operatorname{tp}_{2}\left(\operatorname{cons}\left(y, z s_{1}\right), z s_{2}\right) \\
\vdots
\end{array}\right\}
$$

Unlike $\mathbb{S R}^{\rightarrow}\left(R_{7}\right)$, this unraveled TRS $\mathbb{U}\left(R_{7}\right)$ is not confluent since we have a critical peak, e.g., $\operatorname{tp}_{2}($ nil, cons $(0$, nil $)) \leftarrow_{\mathbb{U}\left(R_{7}\right)}^{*} \operatorname{split}(0$, cons $(0$, nil $)) \rightarrow_{\mathbb{U}\left(R_{7}\right)}^{*} U_{16}($ false, 0,0 , nil, nil, nil $)$ that is not joinable. In this case, we can solve this non-confluence by replacing $\mathrm{U}_{16}$ with $\mathrm{U}_{14}$ since the only difference between the second and third rules of $R_{7}$ is whether le $(x, y)$ reduces to true or false. However, this simple solution is not possible in general.

Example 6.7. Consider the following TRS defining snoc that appends the element to the end of the list, e.g., $\operatorname{snoc}([1,2,3], 4)=[1,2,3,4]$ :

$$
R_{11}=\left\{\begin{array}{c}
\operatorname{snoc}(\mathrm{nil}, y) \rightarrow \operatorname{cons}(y, \operatorname{nil}) \\
\operatorname{snoc}(\operatorname{cons}(x, x s), y) \rightarrow \operatorname{cons}(x, \operatorname{snoc}(x s, y))
\end{array}\right\}
$$

The inversion method in [18] inverts this TRS to the following DCTRS $R_{20}$ :

$$
R_{20}=\left\{\begin{array}{l}
\operatorname{snoc}^{-1}(\operatorname{cons}(y, \operatorname{nil})) \rightarrow \operatorname{tp}_{2}(\text { nil }, y) \\
\operatorname{snoc}^{-1}(\operatorname{cons}(x, y s)) \rightarrow \operatorname{tp}_{2}(\operatorname{cons}(x, x s), y) \Leftarrow \operatorname{snoc}^{-1}(y s) \rightarrow \operatorname{tp}_{2}(x s, y)
\end{array}\right\}
$$

This DCTRS $R_{20}$ is unraveled by $\mathbb{U}$ as follows:

$$
\mathbb{U}\left(R_{20}\right)=\left\{\begin{aligned}
& \vdots \\
& \operatorname{snoc}^{-1}(\operatorname{cons}(x, y s)) \rightarrow \mathrm{U}_{21}\left(\operatorname{snoc}^{-1}(y s), x, y s\right) \\
& \mathrm{U}_{21}\left(\operatorname{tp}_{2}(x s, y), x, y s\right) \rightarrow \operatorname{tp}_{2}(\operatorname{cons}(x, x s), y)
\end{aligned}\right\}
$$

The DCTRS $R_{20}$ is confluent, but the unraveled TRS $\mathbb{U}\left(R_{20}\right)$ is not since we have a critical peak $\mathrm{U}_{21}\left(\operatorname{snoc}^{-1}(\right.$ nil $), x$, nil $) \leftarrow \mathbb{U}\left(R_{20}\right) \cdot \rightarrow_{\mathbb{U}\left(R_{20}\right)} \operatorname{tp}_{2}($ nil,$x)$ that is not joinable. The simple solution described in Example 6.6 cannot solve non-confluence of $\mathbb{U}\left(R_{20}\right)$.

"ground confluence". For this reason, confluence is a soundness condition for the case of arbitrary reduction sequences. 
Finally, we show some properties and a notion related to reachable terms that are helpful to compare the SR transformation with unravelings.

Definition 6.8 ([30, 31]). Let $R$ be a DCTRS over a signature $\mathcal{F}$. For a reachable term $s$ in $\mathcal{T}\left(\mathcal{F}_{\mathbb{S R}(R)}, \mathcal{V}\right)$, we define the set $\mathcal{P}_{o s_{\text {str }}}(s)$ of structural positions for $s$ as follows:

- $\mathcal{P}_{0 s_{\mathrm{str}}}(x)=\{\varepsilon\}$ for $x \in \mathcal{V}$,

- $\mathcal{P}_{\text {os }}$ str $(\{t\})=\left\{1 p \mid p \in \mathcal{P}_{\text {os }}\right.$ str $\left.(t)\right\}$,

- $\mathcal{P}_{o s_{\text {str }}}\left(c\left(t_{1}, \ldots, t_{n}\right)\right)=\left\{i p \mid 1 \leq i \leq n, p \in \mathcal{P}_{\text {os }}\right.$ str $\left.\left(t_{i}\right)\right\}$ for $c \in \mathcal{C}_{R}$, and

- $\mathcal{P}_{o s_{\text {str }}}\left(\bar{f}\left(t_{1}, \ldots, t_{n}, u_{1}, \ldots, u_{n_{f}}\right)\right)=\{\varepsilon\} \cup\left\{i p \mid 1 \leq i \leq n, p \in \mathcal{P}_{o s_{\text {str }}}\left(t_{i}\right)\right\}$ for an $n$-ary defined symbol $f \in \mathcal{D}_{R}$.

Note that $\mathcal{P} s_{\text {str }}$ is well-defined for reachable terms while it is not defined for the symbols $\perp$ and $[\cdot]$.

Example 6.9. Consider the following term related to $\mathbb{S R}^{\rightarrow}\left(R_{7}\right)$ in Example 6.6;

$$
\{\overline{\operatorname{split}}(\mathrm{s}(0), \operatorname{cons}(0, \text { cons }(\mathrm{s}(\mathrm{s}(0)), \text { nil })),[\{\overline{\operatorname{split}}(\mathrm{s}(0), \operatorname{cons}(\mathrm{s}(\mathrm{s}(0)), \text { nil }), \perp, \perp)\}, \perp], \perp)\}
$$

The structural positions of this terms are 1, 1.1, 1.1.1, 1.2, 1.2.1, 1.2.2, 1.2.2.1, 1.2.2.1.1, 1.2.2.1.1.1, and 1.2.2.2.

By definition, structural positions have the following property related to contexts.

Lemma 6.10. Let $R$ be a DCTRS over a signature $\mathcal{F}, t$ be a term in $\mathcal{T}\left(\mathcal{F}_{\mathbb{S R}(R)}, \mathcal{V}\right)$, and $C[]_{p}$ be a one-hole context over $\mathcal{F}_{\mathbb{S R}(R)}$ such that $p \in \mathcal{P}_{\text {os }}$ str $(C[])$. Then, $C[\{t\}] \rightarrow_{\mathbb{R}^{*} \rightarrow(R)}^{*}$ $\{C[t]\}$.

The proof of Lemma 6.10 is omitted since it can be easily proved by induction.

6.3. Relationship between Soundness. In this subsection, we show that if $\mathbb{S R}$ is sound for a DCTRS, then so is $\mathbb{U}$. To this end, as in Section 5, we show that all the derivations of $\mathbb{U}$ on terms over the original signature are included in the derivations of $\mathbb{S R}$.

In rewrite rules obtained from $\mathbb{S R}$, the conditional parts related to the same defined symbol are evaluated in parallel, and thus, the system $\mathbb{S R}(R)$ is more reasonable than the system $\mathbb{U}(R)$. Due to the parallel evaluation of conditional parts, $\mathbb{S R}(R)$ can derive all the reduction sequences of $\mathbb{U}(R)$, and thus, soundness of $\mathbb{S R}$ implies that of $\mathbb{U}$.

Lemma 6.11. Let $R$ be a DCTRS over a signature $\mathcal{F}$. Then, $\phi_{\mathbb{S R}(R)}\left(\rightarrow_{\mathbb{U}(R)}^{*}\right) \subseteq \rightarrow_{\mathbb{R}_{R} \rightarrow(R)}^{*}$ on terms in $\mathcal{T}(\mathcal{F}, \mathcal{V})$.

Proof. The proof can be seen in Appendix A.6.

Due to Lemma 6.11, we obtain the following theorem.

Theorem 6.12. If $\mathbb{S R}$ is sound for a syntactically or strongly DCTRS, then so is $\mathbb{U}$.

Proof. Suppose that $\mathbb{S R}$ is sound for a syntactically or strongly DCTRS $R$. Then, it follows from Lemma 6.11 that $\phi_{\mathbb{S R}(R)}\left(\rightarrow_{\mathbb{U}(R)}^{*}\right) \subseteq \rightarrow_{\mathbb{S R}_{R} \rightarrow(R)}^{*}$. Therefore, it follows from Theorem 6.2 that $\mathbb{U}$ is sound for $R$. 
It is not known whether the converse of Theorem 6.12 holds or not.

Similarly to $\mathbb{U}$, the LL property of DCTRSs is not a soundness condition of $\mathbb{S R}$; Suppose that $\mathbb{S R}$ is sound for LL DCTRSs; Then, it follows from Theorem 6.12 that $\mathbb{U}$ is sound for LL DCTRSs, but $\mathbb{U}$ is not sound for every LL DCTRS (see Example 4.1).

Example 6.13. The DCTRS $R_{6}$ in Example 4.1 is transformed by $\mathbb{S R}^{\rightarrow}$ into the following TRS:

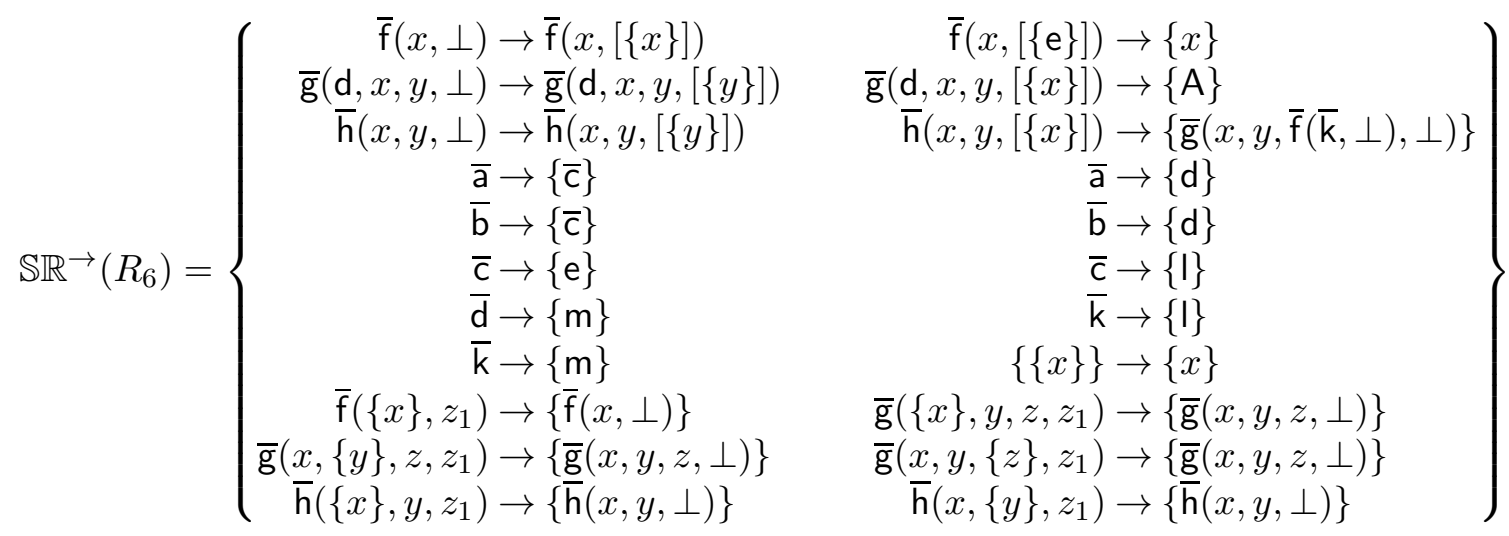

We have the derivation $\overline{\mathrm{h}}(\overline{\mathrm{f}}(\overline{\mathrm{a}}, \perp), \overline{\mathrm{f}}(\overline{\mathrm{a}}, \perp)) \rightarrow_{\mathbb{S R}_{\mathbb{R}} \rightarrow\left(R_{6}\right)}^{*}\{\mathrm{~A}\}$, but $\mathrm{h}(\mathrm{f}(\mathrm{a}), \mathrm{f}(\mathrm{a})) \not \rightarrow_{R_{6}} \mathrm{~A}$. Thus, the LL property is not a sufficient condition for soundness of $\mathbb{S R}$.

6.4. A Comparison from Several Viewpoints. Finally, we compare unravelings with the SR transformation, in terms of the following points.

- Proving Operational Termination. Both the unravelings and the SR transformation can be used for proving operational termination: if the transformed TRS is terminating, then the original CTRS is operationally terminating [14, 30, 31, 28, 29].

- Soundness. As shown in Theorem 6.12, for strongly or syntactically DCTRSs, soundness of $\mathbb{S R}$ implies soundness of $\mathbb{U}$. The known soundness conditions are the $\mathbb{U}_{\text {opt }}-L L$ property and confluence only. These conditions are also the ones for unravelings and more soundness conditions for unravelings are known than those for $\mathbb{S R}$ (see Table 1 in Section (7).

- Strong Soundness. A CTRS transformation $T$ is called strongly sound for an eCTRS $R$ over a signature $\mathcal{F}$ if there exists a (partial) 11 mapping $\psi$ as an inverse to $\phi$ (i.e., $\psi(\phi(t))$ $=t$ for $t \in \mathcal{T}(\mathcal{F}, \mathcal{V})$ ) such that, for all terms $s \in \mathcal{T}(\mathcal{F}, \mathcal{V})$ and $t \in \mathcal{T}(\mathcal{G}, \mathcal{V}), \phi(s) \rightarrow_{R_{T}}^{*} t$ implies $s \rightarrow_{R}^{*} \psi(t)$, where $\mathcal{G}$ is a signature over which $R_{T}$ is defined. The well-designed rules obtained by the SR transformation provide strong soundness from soundness, that plays an important role in the points below. On the other hand, strong soundness of unravelings has never been discussed, and soundness of unravelings does not imply strong soundness of the unravelings in general.

- Proving Confluence. As stated in Theorem 6.5, the SR transformation provides a method for proving confluence of strongly or syntactically U-LL DCTRSs. For unravelings, this has never been discussed, and furthermore, for any overlapping confluent DCTRS, usual

11 The mapping $\psi$ only needs to translate resulting terms (terms reachable from $\phi(s)$ for some original term $s$ ) for $T(R)$ back into the corresponding terms for $R$. 
unravelings (e.g., $\mathbb{U}$ and $\left.\mathbb{U}_{\text {opt }}\right)$ do not preserve confluence, i.e., the unraveled TRS is not confluent (see Examples 6.6, 6.7).

- Computing Normal Forms. For a strongly or syntactically DCTRS $R$, the normal forms of $\mathbb{S R}^{\rightarrow}(R)$ can be converted to the corresponding normal forms of $R$ if $\mathbb{S R}$ is strongly sound for $R$. Thus, $\mathbb{S R}(R)$ can be used for the normalizing reduction of $R$. Moreover, the obtained normal form is a unique one if $R$ is confluent. In general, this is impossible for unravelings.

- Computational Equivalence. For a CTRS transformation $T$ and an eCTRS $R$, the transformed eTRS $R_{T}$ is called computationally equivalent to $R$ if, whenever $R$ terminates on $s$ admitting a unique normal form $t$ (i.e., $t^{\prime}=t$ for all normal forms $t^{\prime}$ of $s$ ), $R_{T}$ also terminates on $\phi(s)$ and for any of its normal forms $t^{\prime}$, we have that $\psi\left(t^{\prime}\right)=t[30$, 31]. $\mathbb{S R}^{\rightarrow}(R)$ is computationally equivalent to $R$ if $R$ is finite, confluent, and operationally terminating [31]. Thus, for such a DCTRS $R, \mathbb{S R}(R)$ can be used as a rewriting engine for $R$ in terms of reduction. This is the main advantage of $\mathbb{S R}$ and has never been discussed for unravelings.

In summary, when the SR transformation is sound for a strongly or syntactically DCTRS with confluence and operational termination, the SR transformation seems better to use as a reasonable rewriting engine for the DCTRS than the unravelings mentioned in this paper. On the other hand, unravelings are good tools for investigating soundness conditions of CTRS transformations, which is required for computational equivalence. Moreover, as stated in Section 1, unravelings are useful in order to analyze or modify DCTRSs. Currently, for DCTRSs that are neither strongly nor syntactically DCTRSs, unravelings are more useful than the SR transformation since it is not known whether $\mathbb{S R}$ provides computational equivalence (and even soundness) for such DCTRSs or not.

\section{Summary and Related Work of Soundness Conditions}

In this section, we briefly describe related work on soundness of unravelings and we summarize positive and negative results on soundness conditions of unravelings and the SR transformation.

First, we briefly describe a comparison with related work, in terms of the approach to the proof of soundness related to the $\mathbb{U}_{\text {opt }}-$ LL property (Subsection 4.1). For an LL normal CTRS $R$ over a signature $\mathcal{F}$, the approach to the proof of soundness in [9] is the use of the transformation $\nabla$ from $\mathcal{T}\left(\mathcal{F}_{\mathbb{U}_{\mathrm{N}}(R)}, \mathcal{V}\right)$ to $\mathcal{T}(\mathcal{F}, \mathcal{V})$, proving that for any term $s \in \mathcal{T}(\mathcal{F}, \mathcal{V})$ and term $t \in \mathcal{T}\left(\mathcal{F}_{\mathbb{U}_{\mathrm{N}}(R)}, \mathcal{V}\right)$, if $s \rightarrow_{\mathbb{U}_{\mathrm{N}}(R)}^{*} t$, then $s \rightarrow_{R}^{*} \nabla(t)[26$. Note that the transformation $\nabla$ has been extended to $\mathbb{U}[25]$ ( $c f .,[26])$. The transformation $\nabla$ was introduced in [25] to discuss innermost termination. Unlike the case of normal CTRSs, however, $\nabla$ has never been used to show soundness. The transformation $\nabla$ cannot be defined well for $\mathbb{U}_{\text {opt }}$ since not all the variables in $l$ appear in $U_{i}^{\rho}\left(t_{i}, \vec{Z}_{i}\right)$. For this reason, the proof in this paper takes a direct approach to the proof of soundness for $\mathbb{U}_{\text {opt }}$ LL DCTRSs ( $c f$. ., Lemma 4.2).

Extending the results in [9], Gmeiner et al. have shown that $\mathbb{U}$ is sound for confluent and right-stable 3-DCTRSs w.r.t. the reduction to normal forms 12 and $\mathbb{U}$ is sound for $\mathbb{U}-\mathrm{RL}$

\footnotetext{
12 A syntactically DCTRS $R$ is called right-stable [32, 10] if for every rule $l \rightarrow r \Leftarrow s_{1} \rightarrow t_{1} ; \ldots ; s_{k} \rightarrow t_{k}$ and for all $1 \leq i \leq n, t_{i}$ is linear and $X_{i} \cap \mathcal{V}$ ar $\left(t_{i}\right)=\emptyset$.
} 
or WLL 3-DCTRSs 10, 13 For the case of U-RL 3-DCTRSs, this result is incompatible with Theorem 4.9 since $\mathbb{U}-\mathrm{RL}$ is strictly more restrictive than $\mathbb{U}_{\text {opt }}-\mathrm{RL}$. For example, the DCTRS

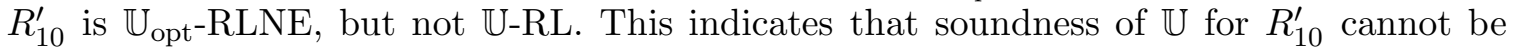
proved by using the result in [10], while the soundness can be proved by the results in this paper (see Example 5.6). For the case of WLL 3-DCTRS, this result strictly contains the combination of Theorem 4.3 and Corollary 5.5 since $\mathbb{U}_{\text {opt }}$-LL 3-DCTRSs are WLL. On the other hand, the WLL property is not a soundness condition of $\mathbb{U}_{\text {opt }}$ since $\mathbb{U}_{\text {opt }}$ is not sound for the WLL DCTRS $R_{10}$ shown in Example 4.12. Furthermore, Gmeiner et al. have also shown that $\mathbb{U}_{\text {opt }}$ is sound for $\mathbb{U}_{\text {opt }}-\mathrm{NE}$ and right-separated 2-DCTRSs 10. 14 The $\mathbb{U}_{\text {opt }}-\mathrm{RL}$ property is incompatible with the right-separated property even if the DCTRSs are $\mathbb{U}_{\text {opt }}-\mathrm{NE}$ and of Type 215 For this reason, the soundness result on $\mathbb{U}_{\text {opt }}$ in $[10$ is incomparable with Theorem 4.9 ,

Finally, we summarize the positive and negative results on soundness in Table 1, i.e., sufficient and insufficient conditions for soundness of the CTRS transformations mentioned in this paper. We can recognize from Example 4.12 that neither confluence nor $\mathbb{U}_{\text {opt}^{-}}$ confluence is sufficient on its own for soundness of $\mathbb{U}_{\text {opt }}$. As we have seen, soundness of the unraveling $\mathbb{U}$ is provided by the other transformations $\mathbb{U}_{\mathrm{J}}, \mathbb{U}_{\mathrm{N}}, \mathbb{U}_{\text {opt }}$, and $\mathbb{S} \mathbb{R}$. In summary, many sufficient and insufficient conditions for soundness of unravelings are investigated and all the soundness conditions of $\mathbb{S R}$ are soundness conditions of unravelings.

\section{Conclusion}

In this paper, we showed that the optimized unraveling for DCTRSs is sound for ultra-LL or ultra-RLNE DCTRSs, and showed that if the optimized unraveling is sound for a DCTRS, then so is Ohlebusch's unraveling. We also presented necessary and sufficient syntactic conditions for ultra-LL, ultra-RL, and ultra-NE, respectively, and soundness conditions of unravelings for join and normal CTRSs. Moreover, we showed that soundness of the existing unravelings and the SR transformation respectively implies soundness of Ohlebusch's unraveling.

Our future work is to solve the remaining open problems, e.g., either to show soundness of $\mathbb{U}$ and $\mathbb{U}_{\text {opt }}$ for $R_{3}^{\prime}$ in Example 5.22 , or to prove the converse of Theorem 5.21. We are also interested in a study on strong soundness and computational equivalence of unravelings.

There seems to be room for discussing sufficient conditions of unravelings related to confluence, e.g., under which confluence of the unraveled TRSs implies that of the original CTRSs, or under which confluence of the original CTRSs implies that of the unraveled TRSs. For a confluent DCTRS $R$, a trivial such condition is that $R$ is $\mathbb{U}_{\text {opt }}-\mathrm{LL}$ and nonoverlapping, i.e., $\mathbb{U}(R)$ and $\mathbb{U}_{\text {opt }}(R)$ are LL and non-overlapping. In many cases, however, neither $\mathbb{U}(R)$ nor $\mathbb{U}_{\text {opt }}(R)$ is confluent even if $R$ is confluent (see Example 6.6). Viewed in

13 A 3-DCTRS $R$ is called weakly left-linear (WLL) [10] if for every rule $\rho: l \rightarrow r \Leftarrow s_{1} \rightarrow t_{1} ; \ldots ; s_{k} \rightarrow t_{k}$ $\in R$ and all variables $x \in \mathcal{V} a r(\rho), x$ does not appear in any of $r, s_{1}, \ldots, s_{k}$ whenever $x$ appears at least twice in $l, t_{1}, \ldots, t_{k}$. Note that this WLL property for 3-DCTRSs is an extension of the WLL property for normal 1-CTRSs.

14 A DCTRS $R$ is called right-separated [10] if for every rule $l \rightarrow r \Leftarrow s_{1} \rightarrow t_{1} ; \ldots ; s_{k} \rightarrow t_{k}$ and all $1 \leq i$ $\leq k, \mathcal{V}$ ar $\left(t_{i}\right) \cap X_{i}=\emptyset$.

15 The rule $\mathrm{f}(x, y, z) \rightarrow x \Leftarrow \mathrm{g}(y) \rightarrow \mathrm{w} ; \mathrm{g}(z) \rightarrow y ; \mathrm{h}(y) \rightarrow$ a is $\mathbb{U}_{\text {opt }}-\mathrm{RLNE}$ and of Type 2 , but not right-separated. On the other hand, the rule $\mathrm{f}(x, y) \rightarrow x \Leftarrow \mathrm{g}(y) \rightarrow z ; \mathrm{g}(z) \rightarrow \mathrm{a} ; \mathrm{h}(z) \rightarrow \mathrm{b}$ is $\mathbb{U}_{\text {opt }}-\mathrm{NE}$, right-separated, and of Type 2 , but not $\mathbb{U}_{\mathrm{opt}}-\mathrm{RLNE}$. 
Table 1: Results on soundness conditions for CTRS transformations.

\begin{tabular}{|c|c|c|c|}
\hline & & soundness & insufficient for soundness \\
\hline $\mathrm{J}$ & $\mathbb{U}_{\mathrm{J}}$ & $\begin{array}{c}\text { LL (Corollary [5.14) } \\
\text { soundness of } \mathbb{U}_{\mathrm{N}} \circ \mathcal{N} \text { orm }(\text { Theorem } 5.13) \\
\text { soundness of } \mathbb{U}_{\mathrm{N}}(\text { Theorem } 5.17) \\
\text { soundness of } \mathbb{U} \circ \text { Det }(\text { Theorem }[5.19]\end{array}$ & \\
\hline $\mathrm{N}$ & $\mathbb{U}_{\mathrm{N}}$ & $\begin{array}{c}\text { LL }\left(=\mathbb{U}_{\text {opt }}-\mathrm{LL}\right)[9] \\
\text { confluence }[9] \\
\text { NE }[9] \\
\text { groundness of all conditions }[9] \\
\text { WLL }(\supset \text { LL) }[9] \\
\text { soundness of } \mathbb{U}_{\mathrm{J}}(\text { Theorem } 5.15)\end{array}$ & $\begin{array}{c}\text { constructor systems [9] } \\
\text { overlay systems [9] } \\
\text { non-RV [9] } \\
\text { RL [9] } \\
\text { overlappingness } 9] \\
\text { UN [9] } \\
\mathrm{UN}^{\rightarrow} \text { [9] }\end{array}$ \\
\hline \multirow{4}{*}{$\mathrm{D}$} & $\mathbb{S R}$ & $\begin{array}{c}\mathbb{U}_{\text {opt }-\mathrm{LL}[31]} \\
\text { confluence }[31]\end{array}$ & LL (Example 6.13) \\
\hline & & confluence and right-stability [10] & confluence [10] \\
\hline & $\mathbb{U}$ & $\begin{array}{c}\mathbb{U}_{\mathrm{opt}^{-}-\mathrm{LL}}[16] \\
\text { soundness of } \mathbb{U}_{\text {opt }}(\text { Corollary }[5.5) \\
\text { soundness of } \mathbb{U}_{\mathrm{N}} \text { (Theorem }[5.21) \\
\text { soundness of } \mathbb{S R}(\text { Theorem }[6.12) \\
\text { U-RL }[10] \\
\text { WLL }\left(\supset \mathbb{U}_{\text {opt }}-\mathrm{LL}\right)[10]\end{array}$ & $\begin{array}{l}\text { confluence [10] } \\
\text { U-NE [10] }\end{array}$ \\
\hline & $\mathbb{U}_{\mathrm{opt}}$ & $\begin{array}{c}\mathbb{U}_{\text {opt }}-\mathrm{LL}(\text { Theorem } 4.3) \\
\mathbb{U}_{\text {opt }}-\mathrm{NE}-\mathrm{RL}(\text { Theorem } 4.9) \\
\mathbb{U}_{\text {opt }^{-N E} \text {, right-separation, and Type } 2[10}\end{array}$ & $\begin{array}{c}\text { LL (Example 4.1) } \\
\mathbb{U}_{\text {opt }}-\mathrm{NE}(\text { Example 4.11) } \\
\text { WLL (Example 4.12) } \\
\mathbb{U}_{\text {opt }}-\mathrm{RL} \text { (Example 4.12) } \\
\text { soundness of } \mathbb{U} \text { (Example 4.12) } \\
\text { confluence (Example 4.12) } \\
\mathbb{U}_{\text {opt-confluence (Example 4.12) }}\end{array}$ \\
\hline
\end{tabular}

- "J", "N", "D", and "S" in the first column represent "join CTRSs", "normal CTRSs", "DCTRSs", and "strongly or syntactically DCTRSs", respectively.

- "soundness of $\mathbb{U}_{N}$ " means that the target is (or can be considered) a normal CTRS and $\mathbb{U}_{N}$ is sound for the target.

- "soundness of $\mathbb{S R}$ " means that the target is a strongly or syntactically DCTRS and $\mathbb{S R}$ is sound for the target.

this light, an interesting further direction related to confluence will be to improve unraveling transformations themselves, e.g., to optimize introduction of $\mathrm{U}$ symbols as stated in Example 6.6. Such an optimization has been already discussed in [30, 31. For unravelings, however, it is not clear what the optimization leads to. What has to be noticed in this direction is that the improvement is in agreement with the SR transformation.

As stated in the comparison with the SR transformation, soundness conditions of unravelings are better studied than soundness of the SR transformation and it must be easier to investigate soundness of unravelings than that of the SR transformation. Thus, it is still worth investigating unravelings while the SR transformation provides a reasonable rewriting engine in terms of computational equivalence to the original CTRSs. On the other 
hand, if the converse of Theorem 6.12 holds, then unravelings would be useful tools to show soundness of the SR transformation. A further direction of this research will be to prove or disprove the converse of Theorem 6.12 .

\section{ACKNOWLEDGEMENTS}

We would like to thank the anonymous reviewers for their kind and useful comments to improve this paper, especially the remark in Footnote 1.

\section{REFERENCES}

[1] S. Antoy, B. Brassel, and M. Hanus. Conditional narrowing without conditions. In Proceedings of the 5th International ACM SIGPLAN Conference on Principles and Practice of Declarative Programming, pages 20-31, ACM Press, 2003.

[2] J. Avenhaus and C. Loría-Sáenz. On conditional rewrite systems with extra variables and deterministic logic programs. In Proceedings of the 5th International Conference on Logic Programming and Automated Reasoning, volume 822 of Lecture Notes in Computer Science, pages 215-229, Springer, 1994.

[3] F. Baader and T. Nipkow. Term Rewriting and All That. Cambridge University Press, United Kingdom, 1998.

[4] J. A. Bergstra and J. W. Klop. Conditional rewrite rules: Confluence and termination. Journal of Computer and System Sciences, 32(3):323-362, 1986.

[5] H. Comon, M. Dauchet, R. Gilleron, F. Jacquemard, D. Lugiez, C. Löding, S. Tison, and M. Tommasi. Tree automata techniques and applications. Available on: http://www.grappa.univ-lille3.fr/tata, 2007. Release October 12th, 2007.

[6] N. Dershowitz and M. Okada. A rationale for conditional equational programming. Theoretical Computer Science, 75(1\&2):111-138, 1990.

[7] F. Durán, S. Lucas, J. Meseguer, C. Marché, and X. Urbain. Proving termination of membership equational programs. In N. Heintze and P. Sestoft, editors, Proceedings of the 2004 ACM SIGPLAN Workshop on Partial Evaluation and Semantics-based Program Manipulation, pages 147-158, ACM, 2004.

[8] E. Giovannetti and C. Moiso. Notes on the elimination of conditions. In S. Kaplan and J.-P. Jouannaud, editors, Proceedings of the 1st International Workshop on Conditional Term Rewriting Systems, volume 308 of Lecture Notes in Computer Science, pages 91-97, Springer, 1987.

[9] K. Gmeiner, B. Gramlich, and F. Schernhammer. On (un)soundness of unravelings. In C. Lynch, editor, Proceedings of the 21st International Conference on Rewriting Techniques and Applications, volume 6 of Leibniz International Proceedings in Informatics, pages 119-134, Schloss Dagstuhl - Leibniz-Zentrum für Informatik, 2010.

[10] K. Gmeiner, B. Gramlich, and F. Schernhammer. On soundness conditions for unraveling deterministic conditional rewrite systems. In A. Tiwari, editor, Proceedings of the 23rd International Conference on Rewriting Techniques and Applications (RTA'12), volume 15 of Leibniz International Proceedings in Informatics, pages 193-208, Schloss Dagstuhl - Leibniz-Zentrum fuer Informatik, May 2012.

[11] J.-M. Hullot. Canonical forms and unification. In W. Bibel and R. A. Kowalski, editors, Proceedings of the 5th International Conference on Automated Deduction, volume 87 of Lecture Notes in Computer Science, pages 318-334, Springer, 1980.

[12] S. Kaplan. Simplifying conditional term rewriting systems: Unification, termination and confluence. Journal of Symbolic Computation, 4(3):295-334, 1987.

[13] S. Lucas. Context-sensitive computations in functional and functional logic programs. Journal of Functional and Logic Programming, 1998(1), 1998.

[14] S. Lucas, C. Marché, and J. Meseguer. Operational termination of conditional term rewriting systems. Information Processing Letters, 95(4):446-453, 2005.

[15] M. Marchiori. Unravelings and ultra-properties. In M. Hanus and M. Rodríguez-Artalejo, editors, Proceedings of the 5th International Conference on Algebraic and Logic Programming, volume 1139 of Lecture Notes in Computer Science, pages 107-121, Springer, 1996. 
[16] M. Marchiori. On deterministic conditional rewriting. Computation Structures Group, Memo 405, MIT Laboratory for Computer Science, 1997.

[17] A. Middeldorp and E. Hamoen. Completeness results for basic narrowing. Applicable Algebra in Engineering, Communication and Computing, 5:213-253, 1994.

[18] N. Nishida. Transformational Approach to Inverse Computation in Term Rewriting. Doctor thesis, Graduate School of Engineering, Nagoya University, Nagoya, Japan, 2004.

[19] N. Nishida and M. Sakai. Completion after program inversion of injective functions. In A. Middeldorp, editor, Proceedings of the 8th International Workshop on Reduction Strategies in Rewriting and Programming, volume 237 of Electronic Notes in Theoretical Computer Science, pages 39-56, Elsevier, 2009.

[20] N. Nishida, M. Sakai, and T. Sakabe. Narrowing-based simulation of term rewriting systems with extra variables and its termination proof. In G. Vidal, editor, Proceedings of the 12th International Workshop on Functional and (Constraint) Logic Programming, volume 86 of Electronic Notes in Theoretical Computer Science, Issue 3, pages 1-18, Elsevier, 2003.

[21] N. Nishida, M. Sakai, and T. Sakabe. On simulation-completeness of unraveling for conditional term rewriting systems. IEICE Technical Report SS2004-18, the Institute of Electronics, Information and Communication Engineers, volume 104, no. 243, pages 25-30, 2004.

[22] N. Nishida, M. Sakai, and T. Sakabe. Partial inversion of constructor term rewriting systems. In J. Giesl, editor, Proceedings of the 16th International Conference on Rewriting Techniques and Applications, volume 3467 of Lecture Notes in Computer Science, pages 264-278, Springer, 2005.

[23] N. Nishida, M. Sakai, and T. Sakabe. Generation of inverse computation programs of constructor term rewriting systems. IEICE Transactions on Information and Systems, J88-D-I(8):1171-1183, 2005 (in Japanese).

[24] N. Nishida, M. Sakai, and T. Sakabe. Soundness of unravelings for deterministic conditional term rewriting systems via ultra-properties related to linearity. In M. Schmidt-Schauß, editor, Proceedings of the 22nd International Conference on Rewriting Techniques and Applications, volume 10 of Leibniz International Proceedings in Informatics, pages 267-282, Schloss Dagstuhl - Leibniz-Zentrum für Informatik, 2011.

[25] E. Ohlebusch. Termination of logic programs: Transformational methods revisited. Applicable Algebra in Engineering, Communication and Computing, 12(1\&2):73-116, 2001.

[26] E. Ohlebusch. Advanced Topics in Term Rewriting. Springer, 2002.

[27] G. Roşu. From conditional to unconditional rewriting. In J. L. Fiadeiro, P. D. Mosses, and F. Orejas, editors, Revised Selected Papers of the 17th International Workshop on Recent Trends in Algebraic Development Techniques, volume 3423 of Lecture Notes in Computer Science, pages 218-233, Springer, 2004.

[28] F. Schernhammer and B. Gramlich. On proving and characterizing operational termination of deterministic conditional rewrite systems. In D. Hofbauer and A. Serebrenik, editors, Proceedings of the 9th International Workshop on Termination, pages 82-85, 2007.

[29] F. Schernhammer and B. Gramlich. Characterizing and proving operational termination of deterministic conditional term rewriting systems. The Journal of Logic and Algebraic Programming, 79(7):659-688, 2010. Revised selected papers of NWPT 2008.

[30] T.-F. Şerbănuţă and G. Roşu. Computationally equivalent elimination of conditions. In F. Pfenning, editor, Proceedings of the 17th International Conference on Rewriting Techniques and Applications, volume 4098 of Lecture Notes in Computer Science, pages 19-34, Springer, 2006.

[31] T.-F. Şerbănuţă and G. Roşu. Computationally equivalent elimination of conditions. Technical Report UIUCDCS-R-2006-2693, University of Illinois at Urbana-Champaign, 2006.

[32] T. Suzuki, A. Middeldorp, and T. Ida. Level-confluence of conditional rewrite systems with extra variables in right-hand sides. In J. Hsiang, editor, Proceedings of the 6th International Conference on Rewriting Techniques and Applications, volume 914 of Lecture Notes in Computer Science, pages 179-193, Springer, 1995.

[33] J. W. Thatcher. Tree Automata: an Informal Survey, chapter 4, pages 143-178, Currents in the Theory of Computing. Prentice Hall, 1973.

[34] Y. Toyama. Confluent term rewriting systems with membership conditions. In S. Kaplan and J.-P. Jouannaud, editors, Proceedings of the 1st International Workshop on Conditional Term Rewriting Systems, volume 308 of Lecture Notes in Computer Science, pages 228-241, Springer, 1987. 
[35] P. Viry. Elimination of conditions. Journal of Symbolic Computation, 28(3):381-401, 1999.

\section{Appendix A. Proofs of Technical Results}

In this appendix, we show missing proofs of some technical results.

\section{A.1. Proof of Theorem 3.8 ,}

Theorem 3.8. Let $\rho: l \rightarrow r \Leftarrow s_{1} \rightarrow t_{1} ; \ldots ; s_{k} \rightarrow t_{k}$ be an extended deterministic conditional rewrite rule. Then, all of the following hold:

(1) $\rho$ is $\mathbb{U}_{\mathrm{opt}}-L L$ iff all of $l, t_{1}, \ldots, t_{k}$ are linear and $\mathcal{V}$ ar $\left(t_{i}\right) \cap X_{i}=\emptyset$ for all $1 \leq i \leq k$,

(2) $\rho$ is $\mathbb{U}_{\mathrm{opt}}-R L$ iff all of $r, s_{1}, \ldots, s_{k}$ are linear and $\mathcal{V}$ ar $\left(s_{i}\right) \cap Y_{i}=\emptyset$ for all $1 \leq i \leq k$, and

(3) $\rho$ is $\mathbb{U}_{\mathrm{opt}}-N E$ iff $\mathcal{V} a r(l) \subseteq \mathcal{V} a r\left(r, s_{1}, \ldots, s_{k}\right)$ and $\mathcal{V} a r\left(t_{i}\right) \subseteq \mathcal{V} a r\left(r, s_{i+1}, \ldots, s_{k}\right)$ for all $1 \leq i \leq k$.

Proof. The case that $\rho$ is unconditional is trivial, so let $k>0$. Recall that $\mathbb{U}_{\text {opt }}(\rho)=$ $\left\{l \rightarrow U_{1}^{\rho}\left(s_{1}, \overrightarrow{Z_{1}}\right), U_{1}^{\rho}\left(t_{1}, \overrightarrow{Z_{1}}\right) \rightarrow U_{2}^{\rho}\left(s_{2}, \overrightarrow{Z_{2}}\right), \ldots, U_{k}^{\rho}\left(t_{k}, \overrightarrow{Z_{k}}\right) \rightarrow r\right\}$.

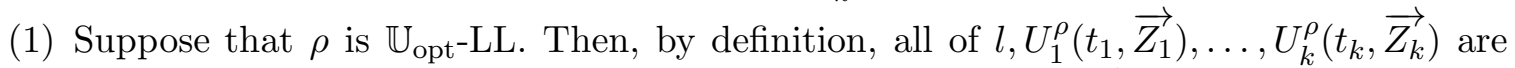
linear. Thus, all of $l, t_{1}, \ldots, t_{k}$ are linear and $\mathcal{V} a r\left(t_{i}\right) \cap Z_{i}=\emptyset$ for all $1 \leq i \leq k$, and hence $\operatorname{Var}\left(t_{i}\right) \cap X_{i}=\emptyset$ for all $1 \leq i \leq k$. Therefore, the only-if part holds.

Suppose that $\rho$ is not $\mathbb{U}_{\text {opt }}-\mathrm{LL}$, all of $l, t_{1}, \ldots, t_{k}$ are linear, and $\mathcal{V}$ ar $\left(t_{i}\right) \cap X_{i}=\emptyset$ for all $1 \leq i \leq k$. Then, $\operatorname{Var}\left(t_{j}\right) \cap Z_{j} \neq \emptyset$ for some $j$ since all of $l, t_{1}, \ldots, t_{k}$ are linear and the sequence $\vec{Z}_{i}$ is linear w.r.t. variable occurrences for all $1 \leq i \leq k$. Since $Z_{i} \subseteq X_{i}$ for all $1 \leq i \leq k$ by definition, we have $\mathcal{V} a r\left(t_{j}\right) \cap X_{j} \neq \emptyset$. This contradicts the assumption that $\mathcal{V} a r\left(t_{i}\right) \cap X_{i}=\emptyset$ for all $1 \leq i \leq k$. Therefore, the if part holds.

(2) Suppose that $\rho$ is $\mathbb{U}_{\text {opt }}-\mathrm{RL}$. Then, by definition, all of $r, U_{1}^{\rho}\left(s_{1}, \overrightarrow{Z_{1}}\right), \ldots, U_{k}^{\rho}\left(s_{k}, \overrightarrow{Z_{k}}\right)$ are linear and $\operatorname{Var}\left(s_{i}\right) \cap Z_{i}=\emptyset$ for all $1 \leq i \leq k$, and hence all of $r, s_{1}, \ldots, s_{k}$ are linear. Since $\rho$ is deterministic, we have $\mathcal{V} a r\left(s_{i}\right) \subseteq X_{i}$, and hence $\mathcal{V} a r\left(s_{i}\right) \cap Y_{i}=\emptyset$ for all $1 \leq$ $i \leq k$. Therefore, the only-if part holds.

Suppose that $\rho$ is not $\mathbb{U}_{\text {opt }}-\mathrm{RL}$, all of $r, s_{1}, \ldots, s_{k}$ are linear, and $\mathcal{V} a r\left(s_{i}\right) \cap Y_{i}=\emptyset$ for all $1 \leq i \leq k$. Then, by definition, $\operatorname{Var}\left(s_{j}\right) \cap Z_{j} \neq \emptyset$ for some $j$ since all of $r, s_{1}, \ldots, s_{k}$ are linear and the variable sequence $\vec{Z}_{i}$ is linear w.r.t. variable occurrences for all $1 \leq$ $i \leq k$. Since $Z_{i} \subseteq Y_{i}$ for all $1 \leq i \leq k$ by definition, it follows from $Z_{j} \subseteq Y_{j}$ that $\mathcal{V} a r\left(s_{j}\right) \cap Y_{j} \neq \emptyset$. This contradicts the assumption that $\mathcal{V}$ ar $\left(s_{i}\right) \cap Y_{i}=\emptyset$ for all $1 \leq i$ $\leq k$. Therefore, the if part holds.

(3) Suppose that $\rho$ is $\mathbb{U}_{\text {opt }}$-NE. Then, by definition, $\operatorname{Var}(l) \subseteq \mathcal{V}$ ar $\left(s_{1}\right) \cup Z_{1}, \mathcal{V} a r\left(t_{i}\right) \cup Z_{i} \subseteq$ $\mathcal{V} a r\left(s_{i+1}\right) \cup Z_{i+1}$ for all $1 \leq i<k$, and $\mathcal{V} a r\left(t_{k}\right) \cup Z_{k} \subseteq \mathcal{V}$ ar $(r)$, and hence $\mathcal{V} a r\left(t_{k}\right) \subseteq$ $\mathcal{V} a r(r), Z_{k} \subseteq \mathcal{V}$ ar $(r), \mathcal{V} a r\left(t_{i}\right) \subseteq \mathcal{V} a r\left(s_{i+1}\right) \cup Z_{i+1}$ and $Z_{i} \subseteq \mathcal{V}$ ar $\left(s_{i+1}\right) \cup Z_{i+1}$ for all $1 \leq$ $i<k$. Thus, $Z_{i} \subseteq \mathcal{V}$ ar $\left(s_{i+1}\right) \cup Z_{i+1} \subseteq \operatorname{Var}\left(s_{i+1}, s_{i+2}\right) \cup Z_{i+2} \subseteq \cdots \subseteq \mathcal{V}$ ar $\left(s_{i+1}, \ldots, s_{k}\right) \cup$ $\mathcal{V} a r(r)$ for all $1 \leq i<k$, and hence $\mathcal{V} a r\left(t_{i}\right) \subseteq \mathcal{V} \operatorname{Var}\left(r, s_{i+1}, \ldots, s_{k}\right)$ for all $1 \leq i \leq k$. Moreover, $\operatorname{Var}(l) \subseteq \mathcal{V}$ ar $\left(s_{1}\right) \cup Z_{1} \subseteq \operatorname{Var}\left(s_{1}\right) \cup \operatorname{Var}\left(r, s_{2}, \ldots, s_{k}\right)=\operatorname{Var}\left(r, s_{1}, \ldots, s_{k}\right)$. Therefore, the only-if part holds.

Suppose that $\mathcal{V} a r(l) \subseteq \mathcal{V} a r\left(r, s_{1}, \ldots, s_{k}\right)$ and $\mathcal{V} a r\left(t_{i}\right) \subseteq \mathcal{V} a r\left(r, s_{i+1}, \ldots, s_{k}\right)$ for all 1 $\leq i \leq k$. Then, by the definition of $Y_{i}$, we have $Y_{i}=\mathcal{V}$ ar $\left(r, s_{i+1}, \ldots, s_{k}\right)$ for all $1 \leq i \leq$ $k$. 
- Consider the rule $U_{k}^{\rho}\left(t_{k}, \overrightarrow{Z_{k}}\right) \rightarrow r \in \mathbb{U}_{\text {opt }}(\rho)$. It follows from $\mathcal{V} a r\left(t_{k}\right) \subseteq \mathcal{V} a r(r)$ and $Y_{k}$ $=\mathcal{V} a r(r)$ that $\operatorname{Var}\left(t_{k}\right) \cup Z_{k}=\mathcal{V} a r\left(t_{k}\right) \cup\left(X_{k} \cap Y_{k}\right) \subseteq \mathcal{V}$ ar $(r)$. Thus, $U_{k}^{\rho}\left(t_{k}, \overrightarrow{Z_{k}}\right) \rightarrow r$ $\in \mathbb{U}_{\text {opt }}(\rho)$ is NE.

- Consider the rule $U_{i}^{\rho}\left(t_{i}, \vec{Z}_{i}\right) \rightarrow U_{i+1}^{\rho}\left(s_{i+1}, \overrightarrow{Z_{i+1}}\right) \in \mathbb{U}_{\text {opt }}(\rho)$ with $1 \leq i<k$. Suppose that $\mathcal{V} a r\left(t_{i}\right) \cup Z_{i} \nsubseteq \mathcal{V} a r\left(s_{i+1}\right) \cup Z_{i+1}$. Then, there exists a variable $x \in \mathcal{V} a r\left(t_{i}\right) \cup Z_{i}$ such that $x \notin \mathcal{V}$ ar $\left(s_{i+1}\right) \cup Z_{i+1}$, and hence $x \notin \mathcal{V}$ ar $\left(s_{i+1}\right) \cup Y_{i+1}$. It follows from $Y_{i+1}$ $=\mathcal{V} a r\left(r, s_{i+2}, \ldots, s_{k}\right)$ that $x \notin \mathcal{V} a r\left(r, s_{i+1}, \ldots, s_{k}\right)$.

- Suppose that $x \in \mathcal{V}$ ar $\left(t_{i}\right)$. Then, it follows from $\mathcal{V} a r\left(t_{i}\right) \subseteq \mathcal{V} a r\left(r, s_{i+1}, \ldots, s_{k}\right)$ that $x \in \mathcal{V} a r\left(r, s_{i+1}, \ldots, s_{k}\right)$. This contradicts the fact that $x \notin \mathcal{V} a r\left(r, s_{i+1}, \ldots, s_{k}\right)$.

- Suppose that $x \notin \mathcal{V} a r\left(t_{i}\right)$. Then, $x \in Z_{i}=X_{i} \cap Y_{i}$, and hence $x \in X_{i}$ and $x \in Y_{i}$ $=\mathcal{V} a r\left(r, s_{i+1}, \ldots, s_{k}\right)$. This contradicts the fact that $x \notin \mathcal{V} a r\left(r, s_{i+1}, \ldots, s_{k}\right)$.

Thus, $U_{i}^{\rho}\left(t_{i}, \vec{Z}_{i}\right) \rightarrow U_{i+1}^{\rho}\left(s_{i+1}, \overrightarrow{Z_{i+1}}\right)$ is NE.

- Consider the remaining rule $l \rightarrow U_{1}^{\rho}\left(s_{1}, \overrightarrow{Z_{1}} \in \mathbb{U}_{\text {opt }}(\rho)\right.$. Suppose that $\operatorname{Var}(l) \nsubseteq$ $\left.\mathcal{V} a r\left(s_{1}\right) \cup Z_{1}\right)$. Then, there exists a variable $x \in \mathcal{V}$ ar $(l)$ such that $x \notin \mathcal{V} a r\left(s_{1}\right) \cup$ $Z_{1}$, and hence $x \notin \mathcal{V} a r\left(s_{1}\right) \cup Y_{1}$. It follows from $Y_{1}=\mathcal{V}$ ar $\left(r, s_{2} \ldots, s_{k}\right)$ that $x \notin$ $\operatorname{Var}\left(r, s_{1}, \ldots, s_{k}\right)$. This contradicts the fact that $\operatorname{Var}(l) \subseteq \mathcal{V}$ ar $\left(r, s_{1}, \ldots, s_{k}\right)$. Thus, $l \rightarrow U_{1}^{\rho}\left(s_{1}, \overrightarrow{Z_{1}}\right)$ is NE.

Therefore, $\mathbb{U}_{\text {opt }}(\rho)$ is NE, and hence the if part holds.

\section{A.2. Proof of Theorem 3.9 ,}

Theorem 3.9. Let $\rho: l \rightarrow r \Leftarrow s_{1} \rightarrow t_{1} ; \ldots ; s_{k} \rightarrow t_{k}$ be an extended deterministic conditional rewrite rule. Then, all of the following hold:

(1) $\rho$ is $\mathbb{U}-L L$ iff all of $l, t_{1}, \ldots, t_{k}$ are linear and $\mathcal{V}$ ar $\left(t_{i}\right) \cap X_{i}=\emptyset$ for all $1 \leq i \leq k$,

(2) $\rho$ is $\mathbb{U}-R L$ iff $r$ is linear and all of $s_{1}, \ldots, s_{k}$ are ground, and

(3) $\rho$ is $\mathbb{U}-N E$ iff $\mathcal{V} a r\left(l, t_{1}, \ldots, t_{k}\right) \subseteq \mathcal{V}$ ar $(r)$.

Proof. The case that $\rho$ is unconditional is trivial, so let $k>0$. Recall that $\mathbb{U}(\rho)=\{l \rightarrow$ $\left.U_{1}^{\rho}\left(s_{1}, \overrightarrow{X_{1}}\right), U_{1}^{\rho}\left(t_{1}, \overrightarrow{X_{1}}\right) \rightarrow U_{2}^{\rho}\left(s_{2}, \overrightarrow{X_{2}}\right), \ldots, U_{k}^{\rho}\left(t_{k}, \overrightarrow{X_{k}}\right) \rightarrow r\right\}$.

(1) This claim can be proved similarly to Theorem 3.8 (1).

(2) Suppose that $\rho$ is $\mathbb{U}$-RL. Then, by definition, all of $r, U_{1}^{\rho}\left(s_{1}, \overrightarrow{X_{1}}\right), \ldots, U_{k}^{\rho}\left(s_{k}, \overrightarrow{X_{k}}\right)$ are linear. Suppose that $s_{j}$ is not ground for some $j$. Then, there exists a variable $x \in$ $\operatorname{Var}\left(s_{j}\right)$. Since $\rho$ is deterministic, $x$ appears in any of $l, t_{1}, \ldots, t_{j-1}$, and hence $x \in$ $X_{j}$. Thus, $U_{i}^{\rho}\left(s_{i}, \overrightarrow{X_{j}}\right)$ is not linear, and hence $\mathbb{U}(\rho)$ is not $\mathrm{RL}$, i.e., $\rho$ is not $\mathbb{U}-\mathrm{RL}$. This contradicts the assumption that $\rho$ is $\mathbb{U}$-RL. Therefore, all of $s_{1}, \ldots, s_{k}$ are ground, and hence the only-if part holds.

Suppose that $\rho$ is not $\mathbb{U}-\mathrm{RL}, r$ is linear, and all of $s_{1}, \ldots, s_{k}$ are ground. Then, by definition, $U_{j}^{\rho}\left(s_{j}, \overrightarrow{X_{j}}\right)$ is not linear for some $j$ since $r$ is linear. It follows from groundness of $s_{j}$ that $\operatorname{Var}\left(s_{j}\right) \cap X_{j}=\emptyset$. Moreover, since the variable sequence $\overrightarrow{X_{j}}$ is linear w.r.t. variable occurrences, the term $U_{j}^{\rho}\left(s_{j}, \overrightarrow{X_{j}}\right)$ is linear. This contradicts the non-linearity of $U_{j}^{\rho}\left(s_{j}, \overrightarrow{X_{j}}\right)$. Therefore, the if part holds.

(3) Suppose that $\rho$ is $\mathbb{U}-\mathrm{NE}$. Then, by definition, the rule $U_{k}^{\rho}\left(t_{k}, \overrightarrow{X_{k}}\right) \rightarrow r$ is NE, and hence $\mathcal{V} a r\left(l, t_{1}, \ldots, t_{k}\right)=\mathcal{V} a r\left(t_{k}\right) \cup X_{k} \subseteq \mathcal{V} a r(r)$. Therefore, the only-if part holds. 
Suppose that $\mathcal{V} a r\left(l, t_{1}, \ldots, t_{n}\right) \subseteq \mathcal{V} a r(r)$. Then, since $X_{k}=\operatorname{Var}\left(l, t_{1}, \ldots, t_{k-1}\right)$ by definition, $U_{k}^{\rho}\left(t_{k}, \overrightarrow{X_{k}}\right) \rightarrow r$ is NE. It follows from $\mathcal{V} a r(l)=X_{1}$ that $l \rightarrow U_{1}^{\rho}\left(s_{1}, \overrightarrow{X_{1}}\right)$ is NE. Since $\mathcal{V}$ ar $\left(t_{i}\right) \cup X_{i}=X_{i+1}$ for all $1 \leq i<k$ by definition, $U_{i}^{\rho}\left(t_{i}, \overrightarrow{X_{i}}\right) \rightarrow U_{i+1}^{\rho}\left(s_{i+1}, \overrightarrow{X_{i+1}}\right)$ is NE for all $1 \leq i<k$. Thus, $\mathbb{U}(\rho)$ is NE, and hence $\rho$ is $\mathbb{U}$-NE. Therefore, the if part holds.

A.3. Proof of Lemma 4.2, We first prepare a technical lemma to help us to prove Lemma 4.2. Let $X$ be a finite set of variables, $\sigma$ and $\theta$ be substitutions, and $\rightarrow$ be a binary relation on terms. Then, we write $X \sigma \rightarrow X \theta$ if $x \sigma \rightarrow x \theta$ for any $x \in X$.

Lemma A.1. Let $R$ be an eDCTRS, $\rho: l \rightarrow r \Leftarrow s_{1} \rightarrow t_{1} ; \ldots ; s_{k} \rightarrow t_{k}$ be a $\mathbb{U}_{\mathrm{opt}}-L L$ conditional rewrite rule in $R$, and $\sigma_{1}, \ldots, \sigma_{k+1}$ be substitutions. If $s_{i} \sigma_{i} \rightarrow_{R}^{*} t_{i} \sigma_{i+1}$ and $Z_{i} \sigma_{i}$ $\rightarrow{ }_{R}^{*} Z_{i} \sigma_{i+1}$ for all $1 \leq i \leq k$, then $l \sigma_{1} \rightarrow_{R}^{+} r \sigma_{k+1}$.

Proof. Let $\sigma$ be the substitution $\left.\left.\left.\left.\sigma_{1}\right|_{\mathcal{V} a r(l)} \cup \sigma_{2}\right|_{Z_{1} \backslash \mathcal{V} a r(l)} \cup \cdots \cup \sigma_{k}\right|_{Z_{k} \backslash Z_{k-1}} \cup \sigma_{k+1}\right|_{\mathcal{V} a r\left(t_{k}, r\right) \backslash Z_{k}}$. Then, $l \sigma=l \sigma_{1}$. It follows from $Z_{i} \sigma_{i} \rightarrow_{R}^{*} Z_{i} \sigma_{i+1}$ that $Z_{i} \sigma \rightarrow_{R}^{*} Z_{i} \sigma_{i+1}$ for all $1 \leq i$ $\leq k$. Moreover, it follows from the $\mathbb{U}_{\text {opt }}-\mathrm{LL}$ property and Theorem 3.8 that $\operatorname{Var}\left(t_{i}\right) \cap$ $\left(\mathcal{D o m}\left(\left.\sigma_{1}\right|_{\mathcal{V a r}(l)}\right) \cup \cdots \cup \mathcal{D} \operatorname{Dom}\left(\left.\sigma_{i-1}\right|_{Z_{i-1} \backslash Z_{i-2}}\right)\right)=\emptyset$ for all $1 \leq i \leq k$, and hence $t_{i} \sigma_{i}=t_{i} \sigma$ for all $1 \leq i \leq k$.

Now we show that $s_{i} \sigma \rightarrow_{R}^{*} s_{i} \sigma_{i}$ for all $1 \leq i \leq k$, i.e., $x \sigma \rightarrow_{R}^{*} x \sigma_{i}$ for all variables $x \in$ $\operatorname{Var}\left(s_{i}\right)$. The case that $i=1$ is trivial, so let $i>1$. We make a case distinction depending on where $x$ appears.

- Consider the case that $x \in \mathcal{V} a r(l)$. By definition, $x \in Z_{j}$ for all $1 \leq j<i$, and hence we have the derivation $x \sigma=x \sigma_{1} \rightarrow_{R}^{*} x \sigma_{2} \rightarrow_{R}^{*} \cdots \rightarrow_{R}^{*} x \sigma_{i}$.

- Consider the remaining case that $x \in \operatorname{Var}\left(t_{j}\right)$ for some $j$ with $1 \leq j<i$. It follows from

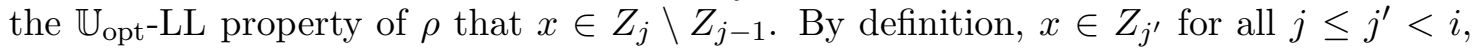
and hence we have the derivation $x \sigma=x \sigma_{j} \rightarrow_{R}^{*} x \sigma_{j+1} \rightarrow_{R}^{*} \cdots \rightarrow_{R}^{*} x \sigma_{i}$.

Thus, $x \sigma \rightarrow_{R}^{*} x \sigma_{i}$ for all variables $x \in \mathcal{V}$ ar $\left(s_{i}\right)$, and hence $s_{i} \sigma \rightarrow_{R}^{*} s_{i} \sigma_{i}$. It follows from the assumption that $s_{i} \sigma \rightarrow_{R}^{*} s_{i} \sigma_{i} \rightarrow_{R}^{*} t_{i} \sigma_{i+1}=t_{i} \sigma$ for all $1 \leq i \leq k$. Similarly, we have the derivation $r \sigma \rightarrow_{R}^{*} r \sigma_{k+1}$. Therefore, we have the derivation $l \sigma_{1}=l \sigma \rightarrow_{R} r \sigma \rightarrow_{R}^{*} r \sigma_{k+1}$.

Next, we show the proof of Lemma 4.2 .

Lemma 4.2, Let $R$ be a $\mathbb{U}_{\text {opt }}-L L$ 3-eDCTRS over a signature $\mathcal{F}, s$ be a term in $\mathcal{T}(\mathcal{F}, \mathcal{V})$, $t$ be a linear term in $\mathcal{T}(\mathcal{F}, \mathcal{V})$, and $\sigma$ be a substitution in $\mathcal{S} u b\left(\mathcal{F}_{\mathbb{U}_{\mathrm{opt}}(R)}, \mathcal{V}\right)$. Suppose that $R$ is non- $L V$ or non- $R V$. If $s \rightrightarrows_{\mathbb{U}_{\mathrm{opt}}(R)}^{n}$ t $\sigma$ for some $n \geq 0$, then there exists a substitution $\theta$ in $\mathcal{S} u b(\mathcal{F}, \mathcal{V})$ such that

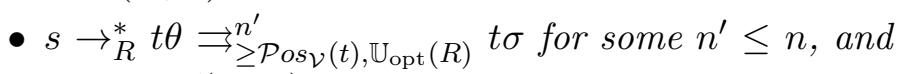

- if $t \sigma \in \mathcal{T}(\mathcal{F}, \mathcal{V})$, then $t \theta=t \sigma$.

Proof. We prove this lemma by induction on the lexicographic product $(n, s)$ of the length $n$ and the structure of $s$. The case that $n=0$ is trivial, so let $n>0$.

We first consider the case that $s \rightrightarrows \mathbb{U}_{\text {opt }}^{n}(R)$ to does not contain any reduction step at the root position. In this case, $s$ is not a variable. Let $s$ be of the form $f\left(s_{1}, \ldots, s_{m}\right)$ with $f \in \mathcal{F}$. We make a case distinction depending on whether $t$ is a variable or not.

- Consider the case that $t$ is not a variable. In this case, $s=f\left(s_{1}, \ldots, s_{m}\right) \rightrightarrows_{\mathbb{U}_{\text {opt }}(R)}^{n}$ $f\left(t_{1}, \ldots, t_{m}\right) \sigma=t \sigma$, and thus, $s_{i} \rightrightarrows_{\mathbb{U}_{\mathrm{opt}}(R)}^{n_{i}} t_{i} \sigma$, where $n_{i} \leq n$, for all $1 \leq i \leq m$. By the 
induction hypothesis, for all $1 \leq i \leq m$, there exists a substitution $\theta_{i} \in \mathcal{S} u b(\mathcal{F}, \mathcal{V})$ such that

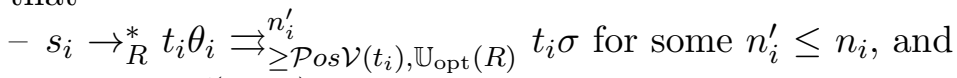

- if $t_{i} \sigma \in \mathcal{T}(\mathcal{F}, \mathcal{V})$, then $t_{i} \theta_{i}=t_{i} \sigma$.

Let $\theta=\left.\left.\theta_{1}\right|_{\mathcal{V} a r\left(t_{1}\right)} \cup \cdots \cup \theta_{m}\right|_{\mathcal{V} a r\left(t_{m}\right)}$. Then, it follows from the linearity of $t$ that $\theta$ is a substitution in $\mathcal{S} u b(\mathcal{F}, \mathcal{V})$. Thus, we have the derivation $s=f\left(s_{1}, \ldots, s_{m}\right) \rightarrow_{R}^{*}$ $f\left(t_{1}, \ldots, t_{m}\right) \theta=t \theta \rightrightarrows \underset{\geq \mathcal{P o s} \mathcal{V}(t), \mathbb{U}_{\mathrm{opt}}(R)}{n^{\prime}}$ t $\sigma$ where

- $n^{\prime}$ is the maximum of $n_{1}^{\prime}, \ldots, n_{m}^{\prime}$, and

- if $t \sigma \in \mathcal{T}(\mathcal{F}, \mathcal{V})$, then $t \theta=t \sigma$.

Moreover, it follows from $n_{i}^{\prime} \leq n$ that $n^{\prime} \leq n$.

- Consider the remaining case that $t$ is a variable $x$. In this case, we can let $\sigma=\{x \mapsto$ $\left.f\left(t_{1}, \ldots, t_{m}\right)\right\}$. Now, let $t^{\prime}$ be a linear term $f\left(x_{1}, \ldots, x_{m}\right)$ with $x_{1}, \ldots, x_{m} \in \mathcal{V}$, and $\sigma^{\prime}=$ $\left\{x_{i} \mapsto t_{i} \mid 1 \leq i \leq m\right\}$. Then, $s=f\left(s_{1}, \ldots, s_{m}\right) \rightrightarrows_{>\varepsilon, \mathbb{U}_{\mathrm{opt}}(R)}^{n} f\left(x_{1}, \ldots, x_{m}\right) \sigma^{\prime}$. Similarly to the previous case, we have a substitution $\theta^{\prime}$ such that

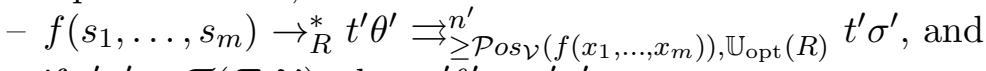

- if $t^{\prime} \sigma^{\prime} \in \mathcal{T}(\mathcal{F}, \mathcal{V})$, then $t^{\prime} \theta^{\prime}=t^{\prime} \sigma^{\prime}$

for some $n^{\prime} \leq n$. Let $\theta=\left\{x \mapsto t^{\prime} \theta^{\prime}\right\}$. Then, we have the derivation $s=f\left(s_{1}, \ldots, s_{m}\right)$ $\rightarrow_{R}^{*} t^{\prime} \theta^{\prime}=t \theta \rightrightarrows \square_{\geq\{\varepsilon\}, \mathbb{U}_{\text {opt }}(R)}^{n^{\prime}} t \sigma$ with $n^{\prime} \leq n$, and $t \theta=t^{\prime} \theta^{\prime}=t \sigma$ whenever $t \sigma \in \mathcal{T}(\mathcal{F}, \mathcal{V})$.

Next we consider the remaining case that at least one rule is applied at the root position. In the following, we make a case distinction depending on whether $R$ is non-LV or non-RV. In the case that $R$ is non-LV, we focus on the first rule applied at the root position, and otherwise (i.e., $R$ is non-RV), we focus on the last rule applied at the root position. The case that the focused rule does not contain a $\mathrm{U}$ symbol is simpler than the other case that the focused rule contains a $U$ symbol since the rule is contained not only in $\mathbb{U}_{\text {opt }}(R)$ but also in $R$. For this reason, we only consider the case that the focused rule contains a $U$ symbol. Now we assume that the focused rule is of the following form:

- $l \rightarrow U_{1}^{\rho}\left(s_{1}, \overrightarrow{Z_{1}}\right)$ if $R$ is non-LV, and

- $U_{i}^{\rho}\left(t_{i}, \vec{Z}_{i}\right) \rightarrow U_{i+1}^{\rho}\left(s_{i+1}, \overrightarrow{Z_{i+1}}\right)$ or $U_{k}^{\rho}\left(t_{k}, \overrightarrow{Z_{k}}\right) \rightarrow r$ if $R$ is non-RV.

For the sake of readability, we assume w.l.o.g. that $k=2$.

Let us start the case distinction mentioned above.

(1) Consider the case that $R$ is non-LV. In this case, we have the following subcases depending on where $t \sigma$ appears.

a. Consider the case that

$$
s \rightrightarrows_{>\varepsilon, \mathbb{U}_{\mathrm{opt}}(R)}^{n_{0}} l \sigma_{1} \rightarrow_{\varepsilon, \mathbb{U}_{\mathrm{opt}}(R)} U_{1}^{\rho}\left(s_{1}, \vec{Z}_{1}\right) \sigma_{1} \rightrightarrows_{>\varepsilon, \mathbb{U}_{\mathrm{opt}}(R)}^{n_{1}} t \sigma
$$

where $n_{0}+n^{\prime \prime}+1=n$. By the induction hypothesis, there exists a substitution $\theta_{1}$

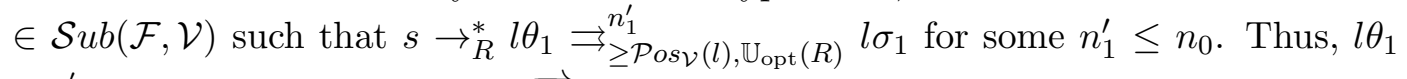
$\rightrightarrows_{\mathbb{U}_{\mathrm{opt}}(R)}^{n^{\prime}} l \sigma_{1} \rightarrow_{\varepsilon, \mathbb{U}_{\mathrm{opt}}(R)} U_{1}^{\rho}\left(s_{1}, \vec{Z}_{1}\right) \sigma_{1} \rightrightarrows_{>\varepsilon, \mathbb{U}_{\mathrm{opt}}(R)}^{n^{\prime \prime}} t \sigma$ with $n_{1}^{\prime}+1+n^{\prime \prime} \leq n$. Since

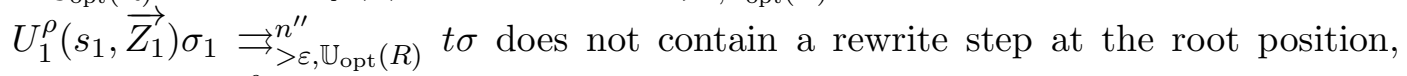
$\operatorname{root}(t \sigma)=U_{1}^{\rho}$. It follows from the assumption $t \in \mathcal{T}(\mathcal{F}, \mathcal{V})$ that $t$ is a variable $x$. Now let $\theta=\left\{x \mapsto l \theta_{1}\right\}$. Then, $\theta$ is a substitution in $\mathcal{S} u b(\mathcal{F}, \mathcal{V})$ such that $s \rightarrow{ }_{R}^{*} l \theta_{1}$ $=t \theta \rightrightarrows \begin{gathered}n_{1}^{\prime}+1+n^{\prime \prime} \\ >\{\varepsilon\}, \mathbb{U}_{\mathrm{opt}}(R)\end{gathered}$ with $n_{1}^{\prime}+1+n^{\prime \prime} \leq n$ and $t \sigma \notin \mathcal{T}(\mathcal{F}, \mathcal{V})$. 
b. Consider the case that

$$
\begin{aligned}
& s \rightrightarrows_{>\varepsilon, \mathbb{U}_{\mathrm{opt}}(R)}^{n_{0}} l \sigma_{1} \rightarrow_{\varepsilon, \mathbb{U}_{\mathrm{opt}}(R)} U_{1}^{\rho}\left(s_{1}, \vec{Z}_{1}\right) \sigma_{1} \rightrightarrows_{>\varepsilon, \mathbb{U}_{\mathrm{opt}}(R)}^{n_{1}} U_{1}^{\rho}\left(t_{1}, \overrightarrow{Z_{1}}\right) \sigma_{2} \\
& \rightarrow_{\varepsilon, \mathbb{U}_{\mathrm{opt}}(R)} U_{2}^{\rho}\left(s_{2}, \vec{Z}_{2}\right) \sigma_{2} \rightrightarrows \underset{>\varepsilon, \mathbb{U}_{\mathrm{opt}}(R)}{n^{\prime \prime}} t \sigma
\end{aligned}
$$

where $n_{0}+n_{1}+n^{\prime \prime}+2=n$. This case is proved similarly to the previous case.

c. Consider the remaining case that

$$
\begin{aligned}
& s \rightrightarrows_{>\varepsilon, \mathbb{U}_{\mathrm{opt}}(R)}^{n_{0}} l \sigma_{1} \rightarrow_{\varepsilon, \mathbb{U}_{\mathrm{opt}}(R)} U_{1}^{\rho}\left(s_{1}, \overrightarrow{Z_{1}}\right) \sigma_{1} \rightrightarrows_{>\varepsilon, \mathbb{U}_{\mathrm{opt}}(R)}^{n_{1}} U_{1}^{\rho}\left(t_{1}, \overrightarrow{Z_{1}}\right) \sigma_{2} \\
& \rightarrow_{\varepsilon, \mathbb{U}_{\mathrm{opt}}(R)} U_{2}^{\rho}\left(s_{2}, \overrightarrow{Z_{2}}\right) \sigma_{2} \rightrightarrows_{>\varepsilon, \mathbb{U}_{\mathrm{opt}}(R)}^{n_{2}} U_{2}^{\rho}\left(t_{2}, \overrightarrow{Z_{2}}\right) \sigma_{3} \\
& \rightarrow_{\varepsilon, \mathbb{U}_{\mathrm{opt}}(R)} r \sigma_{3} \rightrightarrows_{\mathbb{U}_{\mathrm{opt}}(R)}^{n^{\prime \prime}} t \sigma
\end{aligned}
$$

where $n_{0}+n_{1}+n_{2}+n^{\prime \prime}+3=n$. By the induction hypothesis, there exists a substitution $\theta_{1} \in \mathcal{S} u b(\mathcal{F}, \mathcal{V})$ such that $s \rightarrow_{R}^{*} l \theta_{1} \rightrightarrows{\stackrel{P}{\mathcal{P}_{\text {os }}}(l), \mathbb{U}_{\mathrm{opt}}(R)}_{n_{1}^{\prime}} l \sigma_{1}$ for some $n_{1}^{\prime} \leq$ $n_{0}$. Since $l \theta_{1} \rightrightarrows \underset{\geq \mathcal{P}_{\text {os }}(l), \mathbb{U}_{\mathrm{opt}}(R)}{n^{\prime}} l \sigma_{1}$, it follows from the well-known standard property of the parallel reduction [3, Lemma 6.4.2] that $U_{1}^{\rho}\left(s_{1}, \overrightarrow{Z_{1}}\right) \theta_{1} \rightrightarrows \begin{aligned} & n_{1}^{\prime} \\ & \geq \mathcal{P}_{\mathcal{V}}\left(U_{1}^{\rho}\left(s_{1}, \overrightarrow{Z_{1}}\right)\right), \mathbb{U}_{\text {opt }}(R)\end{aligned}$ $U_{1}^{\rho}\left(s_{1}, \overrightarrow{Z_{1}}\right) \sigma_{1}$. Thus, $U_{1}^{\rho}\left(s_{1}, \overrightarrow{Z_{1}}\right) \theta_{1} \rightrightarrows_{>\varepsilon, \mathbb{U}_{\mathrm{opt}}(R)}^{n_{1}^{\prime}} U_{1}^{\rho}\left(s_{1}, \overrightarrow{Z_{1}}\right) \sigma_{1} \rightrightarrows_{>\varepsilon, \mathbb{U}_{\mathrm{opt}}(R)}^{n_{1}} U_{1}^{\rho}\left(t_{1}, \overrightarrow{Z_{1}}\right) \sigma_{2}$ with $n_{1}^{\prime}+n_{1}<n$, and hence $s_{1} \theta_{1} \underset{\mathbb{U}_{\text {opt }}(R)}{n_{1}^{\prime}+n_{1}} t_{1} \sigma_{2}$ and $Z_{1} \theta_{1} \underset{\mathbb{U}_{\mathrm{opt}}(R)}{n_{1}^{\prime}+n_{1}} Z_{1} \sigma_{2}$. Since the $\mathbb{U}_{\text {opt }}-\mathrm{LL}$ property provides the linearity of $t_{1}$, by the induction hypothesis, there exists a substitution $\theta_{2}^{\prime} \in \mathcal{S} u b(\mathcal{F}, \mathcal{V})$ such that $s_{1} \theta_{1} \rightarrow_{R}^{*} t_{1} \theta_{2}^{\prime} \rightrightarrows_{\geq \mathcal{P} \text { os } \mathcal{V}\left(t_{1}\right), \mathbb{U}_{\text {opt }}(R)}^{n^{\prime \prime}} t_{1} \sigma_{2}$ for some $n_{2}^{\prime \prime} \leq n_{1}^{\prime}+n_{1}$. Also, by the induction hypothesis, for any variable $y \in$ $Z_{1}$, there exists a substitution $\delta_{y} \in \mathcal{S} u b(\mathcal{F}, \mathcal{V})$ such that $y \theta_{1} \rightarrow_{R}^{*} y \delta_{y} \rightrightarrows_{\geq\{\varepsilon\}, \mathbb{U}_{\mathrm{opt}}(R)}^{j_{y}}$ $y \sigma_{2}$ for some $j_{y} \leq n_{1}^{\prime}+n_{1}$. Let $\theta_{2}=\theta_{2}^{\prime} \mid \mathcal{V a r}\left(t_{1}\right) \cup\left\{y \mapsto y \delta_{y} \mid y \in Z_{1}\right\}$. Then, since the $\mathbb{U}_{\text {opt }}-\mathrm{LL}$ property provides $\operatorname{Var}\left(t_{1}\right) \cap Z_{1}=\emptyset$, we have the derivations $s_{1} \theta_{1} \rightarrow_{R}^{*}$

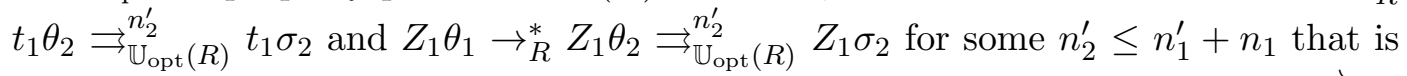
the maximum of $n_{2}^{\prime \prime}$ and $j_{y}$ for $y \in Z_{1}$. Thus, we have the derivation $U_{2}^{\rho}\left(s_{2}, \overrightarrow{Z_{2}}\right) \theta_{2}$ $\rightrightarrows_{>\varepsilon, \mathbb{U}_{\text {opt }}(R)}^{n_{2}^{\prime}+n_{2}} U_{2}^{\rho}\left(t_{2}, \overrightarrow{Z_{2}}\right) \sigma_{3}$ with $n_{2}^{\prime}+n_{2}<n$.

In the same way, we obtain a substitution $\theta_{3}$ in $\mathcal{S} u b(\mathcal{F}, \mathcal{V})$ such that $s_{2} \theta_{2} \rightarrow_{R}^{*} t_{2} \theta_{3}$, $Z_{2} \theta_{2} \rightarrow_{R}^{*} Z_{2} \theta_{3}, U_{2}^{\rho}\left(t_{2}, \overrightarrow{Z_{2}}\right) \theta_{3} \rightrightarrows_{>\varepsilon, \mathbb{U}_{\mathrm{opt}}(R)}^{n_{3}^{\prime}} U_{2}^{\rho}\left(t_{2}, \vec{Z}_{2}\right) \sigma_{3}$ for some $n_{3}^{\prime} \leq n_{2}^{\prime}+n_{2}$. Moreover, in the same way, we obtain a substitution $\theta$ in $\mathcal{S} u b(\mathcal{F}, \mathcal{V})$ such that $r \theta_{3} \rightarrow_{R}^{*}$ $t \theta \rightrightarrows \square_{\geq \mathcal{P}_{\text {os }}(t), \mathbb{U}_{\text {opt }}(R)}^{n^{\prime}} t \sigma$ and $t \sigma \in \mathcal{T}(\mathcal{F}, \mathcal{V})$ implies $t \theta=t \sigma$, where $n^{\prime} \leq n_{3}^{\prime}+n^{\prime \prime}<n$. It follows from Lemma A.1 that $l \theta_{1} \rightarrow_{R}^{+} r \theta_{3}$. Therefore, we have the derivation $s$ $\rightarrow_{R}^{*} l \theta_{1} \rightarrow_{R}^{+} r \theta_{3} \rightarrow_{R}^{*} t \theta \rightrightarrows_{\geq \mathcal{P}_{\text {os }}(t), \mathbb{U}_{\mathrm{opt}}(R)}^{n^{\prime}} t \sigma$ with $n^{\prime} \leq n$, and $t \theta=t \sigma$ whenever $t \sigma \in$ $\mathcal{T}(\mathcal{F}, \mathcal{V})$.

(2) Consider the remaining case (i.e., $R$ is non-LV). Similarly to Case (1), we have the following subcases.

a. Consider the case that

$$
s \rightrightarrows_{\mathbb{U}_{\mathrm{opt}}(R)}^{n_{0}} l \sigma_{1} \rightarrow_{\mathbb{U}_{\mathrm{opt}}(R)} U_{1}^{\rho}\left(s_{1}, \vec{Z}_{1}\right) \sigma_{1} \rightrightarrows_{>\varepsilon, \mathbb{U}_{\mathrm{opt}}(R)}^{n_{1}} t \sigma
$$

where $n_{0}+n^{\prime \prime}+1=n$. The only difference from Case (1)-a is that $s \rightrightarrows_{\mathbb{U}_{\mathrm{opt}}(R)}^{n_{0}} l \sigma_{1}$ may contain a rewrite step at the root position. This case can be proved similarly to Case (1)-a. 
b. Consider the case that

$$
\begin{aligned}
& s \rightrightarrows_{\mathbb{U}_{\mathrm{opt}}(R)}^{n_{0}} l \sigma_{1} \rightarrow_{\varepsilon, \mathbb{U}_{\mathrm{opt}}(R)} U_{1}^{\rho}\left(s_{1}, \overrightarrow{Z_{1}}\right) \sigma_{1} \rightrightarrows_{>\varepsilon, \mathbb{U}_{\mathrm{opt}}(R)}^{n_{1}} U_{1}^{\rho}\left(t_{1}, \overrightarrow{Z_{1}}\right) \sigma_{2} \\
& \rightarrow_{\varepsilon, \mathbb{U}_{\mathrm{opt}}(R)} U_{k}^{\rho}\left(s_{2}, \vec{Z}_{2}\right) \sigma_{2} \rightrightarrows \underset{>\varepsilon, \mathbb{U}_{\mathrm{opt}}(R)}{n^{\prime \prime}} t \sigma
\end{aligned}
$$

where $n_{0}+n_{1}+n^{\prime \prime}+2=n$. Again, the only difference from Case (1)-b is that $s$ $\rightrightarrows_{\mathbb{U}_{\mathrm{opt}}(R)}^{n_{0}} l \sigma_{1}$ may contain a rewrite step at the root position. This case can be proved similarly to Case (1)-b.

c. Consider the remaining case that

$$
\begin{aligned}
s \rightrightarrows \rightrightarrows_{\mathbb{U}_{\mathrm{opt}}(R)}^{n_{0}} l \sigma_{1} & \rightarrow_{\varepsilon, \mathbb{U}_{\mathrm{opt}}(R)} U_{1}^{\rho}\left(s_{1}, \overrightarrow{Z_{1}}\right) \sigma_{1} \rightrightarrows_{>\varepsilon, \mathbb{U}_{\mathrm{opt}}(R)}^{n_{1}} U_{1}^{\rho}\left(t_{1}, \overrightarrow{Z_{1}}\right) \sigma_{2} \\
& \rightarrow_{\varepsilon, \mathbb{U}_{\mathrm{opt}}(R)} U_{k}^{\rho}\left(s_{2}, \overrightarrow{Z_{2}}\right) \sigma_{2} \rightrightarrows_{>\varepsilon, \mathbb{U}_{\mathrm{opt}}(R)}^{n_{2}} U_{k}^{\rho}\left(t_{2}, \overrightarrow{Z_{2}}\right) \sigma_{3} \\
& \rightarrow_{\varepsilon, \mathbb{U}_{\mathrm{opt}}(R)} r \sigma_{3} \rightrightarrows_{>\varepsilon, \mathbb{U}_{\mathrm{opt}}(R)}^{n^{\prime \prime}} t \sigma
\end{aligned}
$$

where $n_{0}+n_{1}+n_{2}+n^{\prime \prime}+3=n$. The only difference from Case (1)-c is that $s \rightrightarrows \mathbb{U}_{\mathbb{o p t}^{\prime}}^{n_{0}}(R)$ $l \sigma_{1}$ may contain a rewrite step at the root position, but $r \sigma_{3} \rightrightarrows_{\mathbb{U}_{\mathrm{opt}}(R)}^{n^{\prime \prime}} t \sigma$ does not contain any rewrite step at the root position. This case can be proved similarly to Case (1)-c.

A.4. Proof of Theorem 4.7. Theorem 4.7 is a direct consequence of the following lemma, a rule-based variant of Theorem 4.7 .

Lemma A.2. Let $\rho: l \rightarrow r \Leftarrow s_{1} \rightarrow t_{1} ; \ldots ; s_{k} \rightarrow t_{k}$ be an (extended) deterministic rewrite rule. Then all of the following hold:

(1) $\mathcal{V} a r\left(t_{i}\right) \subseteq \mathcal{V}$ ar $\left(r, s_{i+1}, \ldots, s_{k}\right)$ for all $1 \leq i \leq k$ iff $(\rho)^{-1}$ is deterministic,

(2) $\operatorname{Var}(l) \subseteq \mathcal{V}$ ar $\left(r, s_{1}, \ldots, s_{k}\right)$ iff $(\rho)^{-1}$ is of Type 3 ,

(3) if $\operatorname{Var}\left(t_{i}\right) \subseteq \mathcal{V}$ ar $\left(r, s_{i+1}, \ldots, s_{k}\right)$ for all $1 \leq i \leq k$, then

a. $\mathbb{U}_{\text {opt }}\left((\rho)^{-1}\right)=\left(\mathbb{U}_{\text {opt }}(\rho)\right)^{-1}$ up to the renaming of $U$ symbols,

b. $\rho$ is $\mathbb{U}_{\mathrm{opt}}-L L$ iff $(\rho)^{-1}$ is $\mathbb{U}_{\mathrm{opt}}-R L$, and

c. $\rho$ is $\mathbb{U}_{\mathrm{opt}}-R L$ iff $(\rho)^{-1}$ is $\mathbb{U}_{\mathrm{opt}}-L L$,

(4) $\rho$ is non- $L V$ iff $(\rho)^{-1}$ is non- $R V$, and

(5) $\rho$ is non-RV iff $(\rho)^{-1}$ is non- $L V$.

Proof. Since the first, second, fourth, and fifth claims are trivial, we only prove the third claim. Recall that $\mathbb{U}_{\text {opt }}(\rho)=\left\{l \rightarrow U_{1}^{\rho}\left(s_{1}, \overrightarrow{Z_{1}}\right), U_{1}^{\rho}\left(t_{1}, \overrightarrow{Z_{1}}\right) \rightarrow U_{2}^{\rho}\left(s_{2}, \overrightarrow{Z_{2}}\right), \ldots, U_{k}^{\rho}\left(t_{k}, \overrightarrow{Z_{k}}\right) \rightarrow\right.$ $r\}$. We assume w.l.o.g. that $\mathbb{U}_{\text {opt }}\left((\rho)^{-1}\right)=\left\{r \rightarrow U_{k}^{\rho}\left(t_{k}, \overrightarrow{V_{k}}\right), \ldots, U_{2}^{\rho}\left(s_{2}, \overrightarrow{V_{2}}\right) \rightarrow U_{1}^{\rho}\left(t_{1}, \overrightarrow{V_{1}}\right)\right.$, $\left.U_{1}^{\rho}\left(s_{1}, \vec{V}_{1}\right) \rightarrow l\right\}$ where $V_{i}=\mathcal{V} a r\left(r, s_{k}, \ldots, s_{i+1}\right) \cap \mathcal{V} a r\left(l, s_{i}, t_{i-1}, s_{i-1}, \ldots, t_{1}, s_{1}\right)$.

Since $\rho$ is deterministic, we have $V_{i}=\mathcal{V} a r\left(r, s_{k}, \ldots, s_{i+1}\right) \cap X_{i}$. Moreover, it follows from the assumption that $Y_{i}=\mathcal{V} a r\left(r, t_{i}, s_{i+1}, t_{i+1}, \ldots, s_{k}, t_{k}\right)=\operatorname{Var}\left(r, s_{i+1}, \ldots, s_{k}\right)$, and hence $V_{i}=X_{i} \cap Y_{i}=Z_{i}$ for all $1 \leq i \leq k$. Therefore, the claim (3)-a holds.

By the definition of $(\cdot)^{-1}, \mathbb{U}_{\text {opt }}(R)$ is LL $(\mathrm{RL})$ iff $\left(\mathbb{U}_{\text {opt }}(R)\right)^{-1}$ is RL (LL). Therefore, the claims (3)-b,c follow from the claim (3)-a. 
A.5. Proof of Lemma 4.15. We first prepare some technical lemmas to prove Lemma 4.15,

Lemma A.3. Let $l$ be a linear term with $U$-symbol-free proper subterms, and $\theta, \sigma, \eta$ be substitutions such that $\theta \in \mathcal{S} u b(\mathcal{F}, \mathcal{V})$ and $\operatorname{root}(x \eta)$ is a $U$ symbol for any $x \in \operatorname{Dom}(\eta)$. If $s \theta \eta=l \sigma$, then there exists a substitution $\sigma^{\prime}$ such that $s \theta=l \sigma^{\prime}$ and $l \sigma=l \sigma^{\prime} \eta$.

Proof (Sketch). This claim can be proved by induction on the term structure of $s$.

Lemma 4.15 is a direct consequence of the following lemma.

Lemma A.4. Let $s$ be a term in $\mathcal{T}\left(\mathcal{F}_{\mathbb{U}_{\text {opt }}(R)}, \mathcal{V}\right)$, $t$ be a term in $\mathcal{T}(\mathcal{F}, \mathcal{V})$, and $\theta, \eta$ be substitutions such that $\theta \in \mathcal{S} u b\left(\mathcal{F}_{\mathbb{U}_{\text {opt }}(R)}, \mathcal{V}\right)$ and $\operatorname{root}(x \eta)$ is a $U$ symbol for any $x \in \operatorname{Dom}(\eta)$. If $\mathcal{P}_{\text {os }}(s \theta): s \theta \eta \overrightarrow{\mathrm{evs}}_{\mathbb{U}_{\mathrm{opt}}(R)}^{*} t$, then there exists a substitution $\sigma \in \mathcal{S} u b(\mathcal{F}, \mathcal{V})$ such that

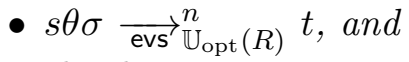

- the derivation is EV-instantiated on $\mathcal{T}(\mathcal{F}, \mathcal{V})$.

Proof. We prove this lemma by induction on the length $n$ of the derivation $\operatorname{Pos}_{\mathcal{F}}(s \theta): s \theta \eta$

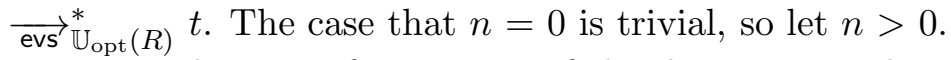

From the EV-safe property of the derivation and Lemma A.3, we can assume w.l.o.g. that

- $s \theta$ is of the form $C\left[s^{\prime}\right]_{p}$ with $s^{\prime}=l \delta$ and $p \in \mathcal{P}_{0 s_{\mathcal{F}}}(s \theta)$,

- $s \theta \eta=C \theta \eta\left[s^{\prime} \eta\right]=C \theta \eta[l \delta \eta]_{p} \overrightarrow{\mathrm{evs}}_{p, l \rightarrow r} C \theta \eta[r \delta \eta]{\underset{\mathrm{evs}}{\mathbb{U}_{\mathrm{opt}}(R)}}^{n-1} t$, and

- $\delta \in \mathcal{S} u b(\mathcal{F}, \mathcal{V})$,

where

- $l \rightarrow r \in \mathbb{U}_{\text {opt }}(R)$ with $\mathcal{V} a r(l, r) \cap \mathcal{V} a r(s \theta)=\emptyset$

- the set $B$ of EV-safe positions in $C \theta \eta[r \delta \eta]$ is $\left(\mathcal{P o s}_{\mathcal{F}}(s \theta) \backslash\left\{q \in \mathcal{P}_{o s}(s \theta) \mid p \leq q\right\}\right) \cup$ $\left\{p q \mid q \in \mathcal{P}_{o s_{\mathcal{F}}}(r)\right\} \cup\left\{p p^{\prime} q\left|p p^{\prime \prime} q \in \mathcal{P}_{o_{\mathcal{F}}}(s \theta), p^{\prime \prime} \in \mathcal{P}_{o s \mathcal{V}}(l), l\right|_{p^{\prime \prime}}=\left.r\right|_{p^{\prime}}\right\}$, and

- $B: C \theta \eta[r \delta \eta] \underset{\text { evs }}{\stackrel{n-1}{\mathbb{U}_{\text {opt }}(R)}} t$.

Let $\delta^{\prime}$ and $\delta^{\prime \prime}$ be substitutions such that $\delta^{\prime} \in \mathcal{S} u b(\mathcal{F}, \mathcal{V}),\left.\delta\right|_{\mathcal{E} \text { Var }(l \rightarrow r)}=\delta^{\prime} \eta, \operatorname{Dom}\left(\delta^{\prime \prime}\right) \cap$ $(\mathcal{V} a r(l, r) \cup \operatorname{Dom}(\eta))=\emptyset$, and $\operatorname{root}\left(x \delta^{\prime \prime}\right)$ is a $\mathrm{U}$ symbol for any $x \in \operatorname{Dom}\left(\delta^{\prime \prime}\right)$.

Let $\theta^{\prime}=\left.\left.\theta\right|_{\mathcal{V} a r(C[])} \cup \delta_{\mathcal{V} a r(l)} \cup \delta^{\prime}\right|_{\mathcal{E} \mathcal{V} a r(l \rightarrow r)}$ and $\eta^{\prime}=\eta \cup \delta^{\prime \prime}$. Then, $\theta^{\prime}$ and $\eta^{\prime}$ are substitutions such that $C \theta \eta[r \delta]=(C[r]) \theta^{\prime} \eta^{\prime}$. It follows from the definition of the EV-safe property that $B=\mathcal{P}_{o s_{\mathcal{F}}}\left((C[r]) \theta^{\prime}\right)$. Thus, by the induction hypothesis, there exists a substitution $\sigma$ in $\mathcal{S} u b(\mathcal{F}, \mathcal{V})$ such that $(C[r]) \theta^{\prime} \sigma \underset{\mathrm{evs}}{\stackrel{n-1}{\mathbb{o p p t}(R)}} t$ and the derivation is EV-instantiated on $\mathcal{T}(\mathcal{F}, \mathcal{V})$. Now, we have the derivation $s \theta \sigma=\left(C \theta\left[s^{\prime}\right]\right) \sigma=(C \theta[l \delta]) \sigma=\left(C \theta^{\prime}\left[l \theta^{\prime}\right]\right) \sigma \underset{\mathrm{evs}}{\overrightarrow{\mathbb{U}_{\text {opt }}}}(R)$ $\left(C \theta^{\prime}\left[r \theta^{\prime}\right]\right) \sigma=(C[r]) \theta^{\prime} \sigma^{\prime} \underset{\text { evs }^{2}}{\mathbb{U}_{\text {opt }}(R)}{ }^{-1}$. Since $\theta$ and $\sigma$ are in $\mathcal{S} u b(\mathcal{F}, \mathcal{V})$, any extra variable in $r$ is instantiated by a term in $\mathcal{T}(\mathcal{F}, \mathcal{V})$. Therefore, this derivation is EV-instantiated on $\mathcal{T}(\mathcal{F}, \mathcal{V})$.

\section{A.6. Proof of Lemma 6.11.}

Lemma 6.11, Let $R$ be a DCTRS over a signature $\mathcal{F}$. Then, $\phi_{\mathbb{S R}(R)}\left(\rightarrow_{\mathbb{U}(R)}^{*}\right) \subseteq \rightarrow_{\mathbb{R}^{*} \rightarrow(R)}^{*}$ on terms in $\mathcal{T}(\mathcal{F}, \mathcal{V})$. 
Proof. We extend the operation - by adding the following clause to the definition of $\bar{\phi}$ in Definition 6.3:

$$
\begin{array}{r}
\bar{\phi}\left(U_{i}^{\rho_{f, j}}\left(x_{i}, \overline{\operatorname{Var}\left(f\left(w_{1}, \ldots, w_{n}\right), t_{1}, \ldots, t_{i-1}\right)}\right)\right. \\
=\bar{f}(\overline{w_{1}}, \ldots, \overline{w_{n}}, \underbrace{\perp, \ldots, \perp}_{j-1},\left[\left\{x_{i}\right\}, \overline{t_{i-1}}, \ldots, \overline{t_{1}}, \perp, \ldots, \perp\right]_{k}, \underbrace{\perp, \ldots, \perp}_{n_{f}-j})
\end{array}
$$

where $\rho_{f, j}: f\left(w_{1}, \ldots, w_{n}\right) \rightarrow r \Leftarrow s_{1} \rightarrow t_{1} ; \ldots ; s_{k} \rightarrow t_{k} \in R, x_{i}$ is a fresh variable, and $\overline{w_{1}}, \ldots, \overline{w_{n}}, \overline{t_{1}}, \ldots, \overline{t_{i-1}}$ are terms obtained by applying the original operation - to $w_{1}, \ldots, w_{n}, t_{1}, \ldots, t_{i-1}$, respectively. We also extend $\phi_{\mathbb{S R}(R)}$ by introducing the extension of $\div$.

To prove this lemma, it suffices to show that for terms $s, t \in \mathcal{T}\left(\mathcal{F}_{\mathbb{U}(R)}, \mathcal{V}\right)$, if $s \rightarrow_{\mathbb{U}(R)}^{*} t$, then $\phi_{\mathbb{S R}(R)}(s) \rightarrow_{\mathbb{S} \mathbb{R} \rightarrow(R)}^{*} \phi_{\mathbb{S R}(R)}(t)$. We prove this claim by induction on the length $m$ of $s$ $\rightarrow_{\mathbb{U}(R)}^{*} t$. The case $m=0$ is trivial, so let $m>0$.

Suppose that $s=C[u \sigma] \rightarrow_{\mathbb{U}(R)} C[v \sigma] \rightarrow_{\mathbb{U}(R)}^{m-1} t$ and $\bar{\sigma}=\{x \mapsto \overline{x \sigma} \mid x \in \operatorname{Dom}(\sigma)\}$. In applying $\phi_{\mathbb{S R}(R)}$ to $C[]$, by definition, the hole in $C[]$ is neither erased nor duplicated. Thus, $\phi_{\mathbb{S R}(R)}(C[])$ is a context of the form $\{\bar{C}\}[]$. Moreover, by definition, the position $p$ of the hole in $\{\bar{C}\}[]$ is structural. Now, we make a case distinction depending on what $u \rightarrow v$ $\in \mathbb{U}(R)$ is.

- Consider the case that $u \rightarrow v$ is $f\left(w_{1}, \ldots, w_{n}\right) \rightarrow r \in R$. Since $\bar{f}\left(\overline{w_{1}}, \ldots, \overline{w_{n}}, z_{1}, \ldots, z_{n_{f}}\right)$ $\rightarrow\{\bar{r}\} \in \mathbb{S R}^{\rightarrow}(R)$ by definition, we have the derivation $\overline{u \sigma}=\bar{f}\left(\overline{w_{1}}, \ldots, \overline{w_{n}}, z_{1}, \ldots, z_{n_{f}}\right) \bar{\sigma}$ $\rightarrow_{\mathbb{S R}} \rightarrow(R)\{\bar{r} \bar{\sigma}\}=\{\overline{v \sigma}\}$.

- Consider the case that $u \rightarrow v$ is $f\left(w_{1}, \ldots, w_{n}\right) \rightarrow U_{1}^{\rho_{f, j}}\left(s_{1}, \overrightarrow{\mathcal{V} a r(l)}\right) \in \mathbb{U}(R)$. By definition, $\overline{u \sigma}=\bar{f}\left(\overline{w_{1}}, \ldots, \overline{w_{n}}, \perp, \ldots, \perp\right) \bar{\sigma}$ and

$$
\begin{aligned}
\bar{f}\left(\overline{w_{1}}, \ldots, \overline{w_{n}}, z_{1}, \ldots, z_{j-1}, \perp, z_{j+1}, \ldots, z_{n_{f}}\right) \rightarrow & \rightarrow \bar{f}\left(\overline{w_{1}}, \ldots, \overline{w_{n}}, z_{1}, \ldots, z_{j-1},\left[\left\{\overline{s_{1}}\right\}, \perp, \ldots, \perp\right], z_{j+1}, \ldots, z_{n_{f}}\right) \in \mathbb{S R}^{\rightarrow}(R) .
\end{aligned}
$$

Therefore, we have the derivation

$$
\begin{aligned}
\overline{u \sigma} & =\bar{f}\left(\overline{w_{1}}, \ldots, \overline{w_{n}}, \perp, \ldots, \perp\right) \bar{\sigma} \\
& \rightarrow \frac{\mathbb{S R}_{\mathbb{R}} \rightarrow(R)}{f}\left(\overline{w_{1}}, \ldots, \overline{w_{n}}, \perp, \ldots, \perp,\left[\left\{\overline{s_{1}}\right\}, \perp, \ldots, \perp\right], \perp, \ldots, \perp\right) \bar{\sigma} \\
& =\overline{U_{1}^{\rho_{f, j}}\left(s_{1}, \overline{\mathcal{V} a r(l)}\right)} \bar{\sigma}=\overline{U_{1}^{\rho_{f, j}}\left(s_{1}, \overline{\operatorname{Var}(l)}\right) \sigma}=\overline{v \sigma} .
\end{aligned}
$$

- Consider the case that $u \rightarrow v$ is the rule $U_{i}^{\rho_{f, j}}\left(t_{i}, \overrightarrow{\mathcal{V} a r\left(l, t_{1}, \ldots, t_{i-1}\right)}\right) \rightarrow U_{i+1}^{\rho_{f, j}}\left(s_{i+1}\right.$, $\left.\overrightarrow{\mathcal{V a r}\left(l, t_{1}, \ldots, t_{i}\right)}\right) \in \mathbb{U}(R)$. By definition,

$$
\overline{u \sigma}=\bar{f}\left(\overline{w_{1}}, \ldots, \overline{w_{n}}, \perp, \ldots, \perp,\left[\left\{\overline{t_{i}}\right\}, \overline{t_{i-1}}, \ldots, \overline{t_{1}}, \perp, \ldots, \perp\right], \perp, \ldots, \perp\right) \bar{\sigma}
$$

and

$$
\begin{gathered}
\bar{f}\left(\overline{w_{1}}, \ldots, \overline{w_{n}}, z_{1}, \ldots, z_{j-1},\left[\left\{\overline{t_{i}}\right\}, \overline{t_{i-1}}, \ldots, \overline{t_{1}}, \perp, \ldots, \perp\right], z_{j+1}, \ldots, z_{n_{f}}\right) \rightarrow \\
\bar{f}\left(\overline{w_{1}}, \ldots, \overline{w_{n}}, z_{1}, \ldots, z_{j-1},\left[\left\{\overline{s_{i+1}}\right\}, \overline{t_{i}}, \ldots, \overline{t_{1}}, \perp, \ldots, \perp\right], z_{j+1}, \ldots, z_{n_{f}}\right) \in \mathbb{S R}^{\rightarrow}(R) .
\end{gathered}
$$

Therefore, we have the derivation

$$
\begin{aligned}
\overline{u \sigma} & =\bar{f}\left(\overline{w_{1}}, \ldots, \overline{w_{n}}, \perp, \ldots, \perp,\left[\left\{\overline{t_{i}}\right\}, \overline{t_{i-1}}, \ldots, \overline{t_{1}}, \perp, \ldots, \perp\right], \perp, \ldots, \perp\right) \bar{\sigma} \\
& \rightarrow \mathbb{S R}^{\rightarrow}(R) \bar{f}\left(\overline{w_{1}}, \ldots, \overline{w_{n}}, \perp, \ldots, \perp,\left[\left\{\overline{s_{i+1}}\right\}, \overline{t_{i}}, \ldots, \overline{t_{1}}, \perp, \ldots, \perp\right], \perp, \ldots, \perp\right) \bar{\sigma} \\
& =\overline{U_{i+1}^{\rho_{f, j}}\left(s_{i+1}, \overline{\operatorname{Var}\left(l, t_{1}, \ldots, t_{i}\right)}\right)} \bar{\sigma}=\overline{U_{i+1}^{\rho_{f, j}}\left(s_{i+1}, \overline{\operatorname{Var}\left(l, t_{1}, \ldots, t_{i}\right.}\right) \sigma}=\overline{v \sigma} .
\end{aligned}
$$


- Consider the remaining case that $u \rightarrow v$ is $U_{k}^{\rho_{f, j}}\left(t_{k}, \overrightarrow{\mathcal{V} a r\left(l, t_{1}, \ldots, t_{k-1}\right)}\right) \rightarrow r \in \mathbb{U}(R)$. By definition,

$$
\overline{u \sigma}=\bar{f}\left(\overline{w_{1}}, \ldots, \overline{w_{n}}, \perp, \ldots, \perp,\left[\left\{\overline{t_{1}}\right\}, \overline{t_{k-1}} \ldots, \overline{t_{1}}\right], \perp, \ldots, \perp\right) \bar{\sigma}
$$

and $\bar{f}\left(\overline{w_{1}}, \ldots, \overline{w_{n}}, z_{1}, \ldots, z_{j-1},\left[\left\{\overline{t_{k}}\right\}, \overline{t_{k-1}}, \ldots, \overline{t_{1}}\right], z_{j+1}, \ldots, z_{n_{f}}\right) \rightarrow\{\bar{r}\} \in \mathbb{S R}^{\rightarrow}(R)$. Therefore, we have the derivation $\overline{u \sigma}=\bar{f}\left(\overline{w_{1}}, \ldots, \overline{w_{n}}, \perp, \ldots, \perp,\left[\left\{\overline{t_{1}}\right\}, \overline{t_{k-1}} \ldots, \overline{t_{1}}\right], \perp, \ldots, \perp\right) \bar{\sigma}$ $\rightarrow_{\mathbb{S R}} \rightarrow(R)\{\bar{r}\} \bar{\sigma}=\{\overline{r \sigma}\}=\{\overline{v \sigma}\}$.

Now, we have either $\overline{u \sigma} \rightarrow_{\mathbb{S R}^{\rightarrow}(R)}\{\overline{v \sigma}\}$ or $\overline{u \sigma} \rightarrow_{\mathbb{S R}^{\rightarrow}(R)} \overline{v \sigma}$.

- Consider the case that $\overline{u \sigma} \rightarrow_{\mathbb{S R}_{\mathbb{R}} \rightarrow(R)}\{\overline{v \sigma}\}$. Since $p$ is a structural position, it follows from Lemma 6.10 that $\phi_{\mathbb{S R}(R)}(s)=\{\overline{C[u \sigma]}\}=\{\bar{C}\}[\overline{u \sigma}] \rightarrow_{\mathbb{S R}_{R} \rightarrow(R)}\{\bar{C}\}[\{\overline{v \sigma}\}] \rightarrow_{\mathbb{S R}^{*}(R)}^{*}$ $\{\{\bar{C}\}[\overline{v \sigma}]\}=\{\{\overline{C[v \sigma]}\}\} \rightarrow_{\mathbb{S R}_{\mathbb{R}} \rightarrow(R)}\{\overline{C[v \sigma]}\}=\phi_{\mathbb{S R}(R)}(C[v \sigma])$.

- Consider the remaining case that $\overline{u \sigma} \rightarrow_{\mathbb{S R}_{R} \rightarrow(R)} \overline{v \sigma}$. Then, $\phi_{\mathbb{S R}(R)}(s)=\{\overline{C[u \sigma]}\}=\{\bar{C}\}[\overline{u \sigma}]$ $\rightarrow_{\mathbb{S R}^{\rightarrow}(R)}\{\bar{C}\}[\overline{v \sigma}]=\{\overline{C[v \sigma]}\}=\phi_{\mathbb{S R}(R)}(C[v \sigma])$.

By the induction hypothesis, $\phi_{\mathbb{S R}(R)}(C[v \sigma]) \rightarrow_{\mathbb{S R}_{R} \rightarrow(R)}^{*} \phi_{\mathbb{S R}(R)}(t)$. Therefore, we have the derivation $\phi_{\mathbb{S R}(R)}(s) \rightarrow_{\mathbb{S R}_{\mathbb{R}} \rightarrow(R)}^{*} \phi_{\mathbb{S R} \rightarrow(R)}(C[v \sigma]) \rightarrow_{\mathbb{S R}_{\mathbb{R}} \rightarrow(R)}^{*} \phi_{\mathbb{S R}(R)}(t)$. 